\title{
Zero Mach Number Limit of the Compressible Primitive Equations: Well-Prepared Initial Data
}

\author{
XIN LiU(1) \& EDRISS S. Titi \\ Communicated by P. Constantin
}

\begin{abstract}
This work concerns the zero Mach number limit of the compressible primitive equations. The primitive equations with the incompressibility condition are identified as the limiting equations. The convergence with well-prepared initial data (i.e., initial data without acoustic oscillations) is rigorously justified, and the convergence rate is shown to be of order $\mathcal{O}(\varepsilon)$, as $\varepsilon \rightarrow 0^{+}$, where $\varepsilon$ represents the Mach number. As a byproduct, we construct a class of global solutions to the compressible primitive equations, which are close to the incompressible flows.
\end{abstract}

\section{Contents}

1. Introduction . . . . . . . . . . . . . . . . . . 705

1.1. The Compressible Primitive Equations . . . . . . . . . . . . . . . 705

1.2. The Low Mach Number Limit Problem and Main Theorem . . . . . . . . . 709

2. Preliminaries . . . . . . . . . . . . . . . . . . . . . . 714

3. $\varepsilon$-Independent A Priori Estimate . . . . . . . . . . . . . . . . . 715

3.1. Temporal Derivatives . . . . . . . . . . . . . . . . 716

3.2. Horizontal Derivatives ． . . . . . . . . . . . . . . . . 722

3.3. Vertical Derivatives Estimates . . . . . . . . . . . . . . . . . . . 725

3.4. Mixed Horizontal and Vertical Derivatives Estimates _ . . . . . . . . . . 727

3.5. Proof of Proposition 1 . . . . . . . . . . . . . . . . . . . . . . . . 727

4. Low Mach Number Limit . . . . . . . . . . . . . . . . . . . . . . . . 729

5. Global Regularity Estimates of the Solution to the Primitive Equations . . . . . 733

References . . . . . . . . . . . . . . . . . . . . . 743

\section{Introduction}

\subsection{The Compressible Primitive Equations}

The compressible primitive equations [see (CPE)] are used by meteorologists to perform theoretical investigations and practical weather predictions (see, e.g., [45]). 
In comparison with the general hydrodynamic and thermodynamic equations, the vertical component of the momentum equations is missing in the compressible primitive equations. Instead, it is replaced by the hydrostatic balance equation [see $\left.(\mathrm{CPE})_{3}\right]$, which is also known as the quasi-static equilibrium equation. From the meteorologists' point of view, such an approximation is reliable and useful for two reasons: the balance of gravity and pressure dominates the dynamics in the vertical direction, and the vertical velocity is usually hard to observe in reality (see, e.g., [59, Chapter 4]). On the other hand, by formally taking the zero limit of the aspect ratio between the vertical scale and the planetary horizontal scale, the authors in [22] derive the compressible primitive equations from the compressible hydrodynamic equations. Such a deviation is very common in planetary scale geophysical models, which represents the fact that the vertical scale of the atmosphere (or ocean) is significantly smaller than the planetary horizontal scale. We refer, for more comprehensive meteorological studies, to [59,66].

As far as we know, there are very few mathematical studies concerning the compressible primitive equations (referred to as CPE hereafter). Lions, Temam, and Wang first introduced CPE into the mathematical community in [45]. They formulated the commonly known primitive equations (referred to as PE hereafter) with the incompressibility condition as the representation of the compressible primitive equations in the pressure coordinates ( $p$-coordinates) instead of the physical ones with the vertical spatial coordinate. On the other hand, as mentioned before, the authors in [22] introduce these equations with a formal deviation, and a rigorous justification is still an open question for now. In [22], the stability of weak solutions is also investigated (see also [63]). The stability is meant in the sense that a sequence of weak solutions, satisfying some entropy conditions, contains a subsequence converging to another weak solution, i.e., a very weak sense of stability. The existence of such weak solutions is recently constructed in $[49,65]$ (see also $[21,30]$ for the existence of global weak solutions to some variant of compressible primitive equations in two spatial dimension). In [50], we also construct local strong solutions to $\mathrm{CPE}$ in two cases: with gravity but no vacuum; with vacuum but no gravity.

In analogy to the low Mach number limit in the study of compressible hydrodynamic equations, this and our subsequent works are aiming to study the low Mach number limit of the compressible primitive equations without gravity and Coriolis force. It is worth mentioning that while taking into account the Coriolis force would not change much our proof, considering gravity in our system causes challenging difficulties. Let $\varepsilon$ denote the Mach number, and let $\rho^{\varepsilon} \in \mathbb{R}, v^{\varepsilon} \in \mathbb{R}^{2}, w^{\varepsilon} \in \mathbb{R}$ be the density, the horizontal and the vertical velocities, respectively. System (CPE), is obtained by rescaling the original $\mathrm{CPE}$, which is similar to the rescaling of the compressible Navier-Stokes equations (see, e.g., [27]): in $\Omega_{h} \times 2 \mathbb{T}$ :

$$
\left\{\begin{array}{l}
\partial_{t} \rho^{\varepsilon}+\operatorname{div}_{h}\left(\rho^{\varepsilon} v^{\varepsilon}\right)+\partial_{z}\left(\rho^{\varepsilon} w^{\varepsilon}\right)=0, \\
\partial_{t}\left(\rho^{\varepsilon} v^{\varepsilon}\right)+\operatorname{div}_{h}\left(\rho^{\varepsilon} v^{\varepsilon} \otimes v^{\varepsilon}\right)+\partial_{z}\left(\rho^{\varepsilon} w^{\varepsilon} v^{\varepsilon}\right) \\
\quad+\frac{1}{\varepsilon^{2}} \nabla_{h} P\left(\rho^{\varepsilon}\right)=\operatorname{div}_{h} \mathbb{S}\left(v^{\varepsilon}\right)+\partial_{z z} v^{\varepsilon}, \\
\partial_{z} P\left(\rho^{\varepsilon}\right)=0,
\end{array}\right.
$$


where $P\left(\rho^{\varepsilon}\right)=\left(\rho^{\varepsilon}\right)^{\gamma}$ and $\mathbb{S}\left(v^{\varepsilon}\right)=\mu\left(\nabla_{h} v^{\varepsilon}+\nabla_{h}^{\top} v^{\varepsilon}\right)+(\lambda-\mu) \operatorname{div}_{h} v^{\varepsilon} \mathbb{I}_{2}$ represent the pressure potential and the viscous stress tensor, respectively, with the shear and bulk viscosity coefficients $\mu$ and $\lambda-\mu+\frac{2}{3} \mu=\lambda-\frac{1}{3} \mu$. The physical requirements of $\mu, \lambda, \gamma$ are $\lambda-\frac{1}{3} \mu>0, \mu>0$ and $\gamma>1$. Moreover, we focus our study on the case when $\Omega_{h}:=\mathbb{T}^{2} \subset \mathbb{R}^{2}$, and we study (CPE) subject to the following symmetry:

$v^{\varepsilon}$ and $w^{\varepsilon}$ are even and odd, respectively, in the $z$-variable.

(SYM-CPE)

Consequently, solutions to (CPE) satisfy the following physical stress-free and impermeability physical boundary conditions:

$$
\left.\partial_{z} v^{\varepsilon}\right|_{z=0,1}=0,\left.w^{\varepsilon}\right|_{z=0,1}=0 .
$$

Hereafter, we have and will use $\nabla_{h}, \operatorname{div}_{h}$ and $\Delta_{h}$ to represent the horizontal gradient, the horizontal divergence, and the horizontal Laplace operator, respectively; that is,

$$
\nabla_{h}:=\left(\begin{array}{c}
\partial_{x} \\
\partial_{y}
\end{array}\right), \operatorname{div}_{h}:=\nabla_{h}, \Delta_{h}:=\operatorname{div}_{h} \nabla_{h}
$$

We recall the incompressible primitive equations: in $\Omega_{h} \times 2 \mathbb{T}$,

$$
\left\{\begin{array}{l}
\operatorname{div}_{h} v_{p}+\partial_{z} w_{p}=0 \\
\rho_{0}\left(\partial_{t} v_{p}+v_{p} \cdot \nabla_{h} v_{p}+w_{p} \partial_{z} v_{p}\right)+\nabla_{h}\left(c_{s}^{2} \rho_{1}\right) \\
\quad=\mu \Delta_{h} v_{p}+\lambda \nabla_{h} \operatorname{div}_{h} v_{p}+\partial_{z z} v_{p} \\
\partial_{z}\left(c_{s}^{2} \rho_{1}\right)=0
\end{array}\right.
$$

subject to the following symmetry:

$v_{p}$ and $w_{p}$ are even and odd respectively in the $z$ variable.

(SYM-PE)

We will show that the asymptotic system of (CPE), as $\varepsilon \rightarrow 0^{+}$, is the incompressible primitive equations (PE), with $c_{s}^{2}=\gamma \rho_{0}^{\gamma-1}$ and $\rho_{0}=$ constant. Here $\rho_{1}$ is the Lagrangian multiplier for the constraint $(\mathrm{PE})_{1}$ satisfying

$$
\int_{\Omega_{h} \times 2 \mathbb{T}} \rho_{1} \mathrm{~d} \vec{x}=0 .
$$

In addition, due to the conservation of linear momentum of (PE), we can impose the following condition for $v_{p}$ :

$$
\int_{\Omega_{h} \times 2 \mathbb{T}} v_{p} d x d y d z=0,
$$

for any time $t \geqq 0$ as long as the solution exists.

Historically, the limit system (PE), besides acting as the representation of the $\mathrm{CPE}$ in the $p$-coordinates, is introduced as the limit system of Boussinesq equations (referred to as BE hereafter) when the aspect ratio between the vertical scale and the horizontal scale is very small, while the Boussinesq equations are the limit equations 


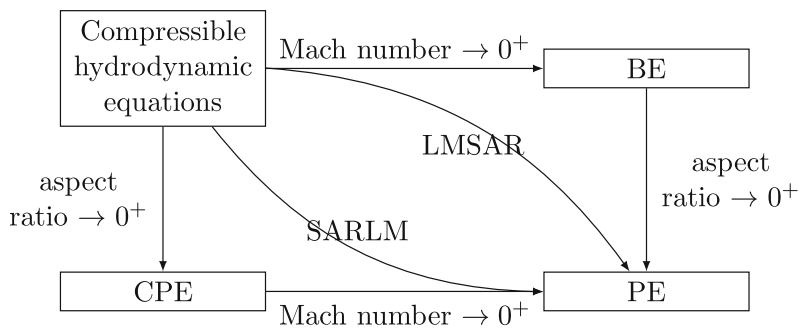

Fig. 1. The PE diagram

of the full compressible hydrodynamic equations with small Mach number and low stratification (see, e.g., [44]). That is to say, starting from the compressible hydrodynamic equations, by taking the low Mach number limit and then the small aspect ratio limit (referred to as LMSAR), one will arrive, formally, at the BE and then at the PE. On the other hand, by taking the small aspect ratio limit and then the low Mach number limit (referred to as SARLM), at least formally, the limit system of the compressible hydrodynamic equations is also the PE with the CPE as a middle state. Depending on the order of asymptotic limits, this gives us two directions from the hydrodynamic equations to the PE, which we will refer to as the PE diagram (see Fig. 1). The LMSAR part of the PE diagram has been shown to hold on solid ground in various settings (see, e.g., [3,28,41,55,57]). However, the validity of the SARLM part is relatively open. In order to fully justify the PE diagram, we investigate the low Mach number limit of the CPE in this work, which, as mentioned above, leads to the PE as the limit system. We remark that, the stratification effect of the gravity has been neglected in this work.

Each of the equations in the PE diagram has its own significance and has been studied separately in a large amount of the literature. It would certainly be too ambitious to review all of those works. We refer readers to the study of compressible hydrodynamic equations in, e.g., the books [23,47,48,54]. As the limit system of the PE diagram, the primitive equations (PE) have been investigated intensively since they were introduced in [43-45]. For instance, the global weak solutions are established in [44]. Local well-posedness with general data and global wellposedness with small data of strong solutions to the PE in three spatial dimensions were studied in [33] by Guillén-González, Masmoudi and Rodríguez-Bellido. Petcu and Wirosoetisno in [56] investigated the Sobolev and Gevrey regularity of the solutions to the PE. In [36], in a domain with small depth, the authors address the global existence of strong solutions to PE. The well-posedness of unique global strong solutions was obtained by Cao and Titi in [12] (see, also, [7-11, 13, 15, 31,34] and the references therein for related studies). Considering the inviscid primitive equations, or hydrostatic incompressible Euler equations, the existence of solutions in the analytic function space and in the $H^{s}$ space are established in $[5,40,53]$. Renardy in [58] showed that the linearization of the equations at certain shear flows is ill-posed in the sense of Hadamard. Recently, the authors in $[6,67]$ constructed a finite-time blowup for the inviscid PE in the absence of rotation (i.e., without the Coriolis force). 
In this work, we show that the PE can be viewed as the limit system of the CPE with the zero Mach number limit. The zero Mach number limit of the compressible hydrodynamic equations is a vast subject which has been studied for decades. Fruitful results have been obtained since the early works of Klainerman and Majda in $[38,39]$, where the authors investigate the vanishing Mach number limit of compressible Euler equations with well-prepared initial data (see also [61,62]). Later Ukai [64], the theory of low Mach number limit of compressible Euler equations extended to ill-prepared initial data (or called general data in some literatures). We remark here that the difference between the well-prepared and ill-prepared initial data is that the well-prepared initial data have excluded the acoustic waves, while the ill-prepared initial data allow the interaction of the solutions with the highfrequency acoustic waves. In $\mathbb{R}^{n}, n=2,3$, such high-frequency acoustic waves disperse as shown in [64], which implies strong convergences as the Mach number goes to zero. This can be also proved by applying Strichartz's estimate (see, e.g., $[4,14,32,37,42])$ for linear wave equations to the acoustic equations (see, e.g., [20]). In $\mathbb{T}^{n}, n=2,3$, the high-frequency acoustic waves interact with each other and lead to fast oscillations and weak convergences when taking the low Mach number limit. Such a fast oscillation phenomenon was first systematically studied in $[29,60]$ for hyperbolic and parabolic systems, and by Lions and Masmoudi for compressible Navier-Stokes equations in [46]. We refer, for the comparison of the whole space case, i.e., in $\mathbb{R}^{n}$ and the periodic domain case, i.e., in $\mathbb{T}^{n}$, to [52]. See also [16-18] for the studies in the Besov spaces. We acknowledge that the discussion here barely unveils the theory of the low Mach number limit, and we refer the reader to $[1,2,19,24-28,55]$ and the references therein for more comprehensive studies and recent progress.

In this work, we will focus on investigating the low Mach number limit of (CPE) with well-prepared initial data. The convergence of the solutions of the CPE to the solution of the PE is in the strong sense. Furthermore, we are also able to obtain explicit convergence rate (see Theorem 1.2). In particular, we obtain a class of global large solutions to (CPE) with $\varepsilon$ small enough.

We remark that in an upcoming paper [51], we will consider the low Mach number limit of (CPE) with ill-prepared initial data, i.e., initial data with large, high-frequency acoustic waves.

\subsection{The Low Mach Number Limit Problem and Main Theorem}

In order to describe the aforementioned asymptotic limit, we study (CPE) with $\left(\rho^{\varepsilon}, v^{\varepsilon}, w^{\varepsilon}\right)$ close to an asymptotic state $\left(\rho_{0}, v_{p}, w_{p}\right)$, where $\left(\rho_{0}, v_{p}, w_{p}\right)$ satisfies (PE). For any $\varepsilon>0$, the following ansatz is imposed:

$$
\left\{\begin{array}{l}
\rho^{\varepsilon}:=\rho_{0}+\varepsilon^{2} \rho_{1}+\xi^{\varepsilon} \\
v^{\varepsilon}:=v_{p}+\psi^{\varepsilon, h} \\
w^{\varepsilon}:=w_{p}+\psi^{\varepsilon, z}
\end{array}\right.
$$

Recall that $\rho_{1}$ has zero average in the domain [see (1.1)]. This is motivated by [35]. The term $\varepsilon^{2} \rho_{1}$ is to capture the cancelation in system (1.4). In addition, we shall 
employ the notation

$$
\zeta^{\varepsilon}:=\varepsilon^{2} \rho_{1}+\xi^{\varepsilon}=\rho^{\varepsilon}-\rho_{0} .
$$

For the sake of convenience, from time to time hereafter, we may drop the superscript $\varepsilon$ from the functions. Consequently, from (CPE) and (PE), the new unknown $\left(\xi, \psi^{h}, \psi^{z}\right)$ is governed by the following system:

$$
\begin{cases}\partial_{t} \xi+\rho_{0}\left(\operatorname{div}_{h} \psi^{h}+\partial_{z} \psi^{z}\right)=-\left(\operatorname{div}_{h}(\xi v)+\partial_{z}(\xi w)\right) & \\ \quad-\varepsilon^{2}\left(\partial_{t} \rho_{1}+\operatorname{div}_{h}\left(\rho_{1} v\right)+\partial_{z}\left(\rho_{1} w\right)\right) & \text { in } \Omega_{h} \times 2 \mathbb{T}, \\ \rho \partial_{t} \psi^{h}+\rho v \cdot \nabla_{h} \psi^{h}+\rho w \partial_{z} \psi^{h}+\nabla_{h}\left(\varepsilon^{-2}\left(\rho^{\gamma}-\rho_{0}^{\gamma}-\varepsilon^{2} c_{s}^{2} \rho_{1}\right)\right) & \\ \quad=\mu \Delta_{h} \psi^{h}+\lambda \nabla_{h} \operatorname{div}_{h} \psi^{h}+\partial_{z z} \psi^{h}+\rho_{0}^{-1}\left(\varepsilon^{2} \rho_{1}+\xi\right) & \\ \quad \times\left(\nabla_{h}\left(c_{s}^{2} \rho_{1}\right)-\mu \Delta_{h} v_{p}-\lambda \nabla_{h} \operatorname{div}_{h} v_{p}-\partial_{z z} v_{p}\right) & \\ \quad-\rho \psi^{h} \cdot \nabla_{h} v_{p}-\rho \psi^{z} \partial_{z} v_{p} & \text { in } \Omega_{h} \times 2 \mathbb{T}, \\ \partial_{z} \xi=0 & \text { in } \Omega_{h} \times 2 \mathbb{T} .\end{cases}
$$

Observe that, owing to the symmetry in (SYM-CPE) and (SYM-PE), the following conditions hold automatically:

$$
\left.\left(\partial_{z} v, \partial_{z} v_{p}, \partial_{z} \psi^{h}\right)\right|_{z \in \mathbb{Z}}=0,\left.\left(w, w_{p}, \psi^{z}\right)\right|_{z \in \mathbb{Z}}=0,
$$

for smooth enough functions. Recalling that $c_{s}^{2}=\gamma \rho_{0}^{\gamma-1}$, we note that

$$
\begin{aligned}
\rho^{\gamma}-\rho_{0}^{\gamma}-\varepsilon^{2} c_{s}^{2} \rho_{1} & =\gamma \rho_{0}^{\gamma-1}\left(\rho-\rho_{0}\right)+\gamma(\gamma-1) \int_{\rho_{0}}^{\rho}(\rho-y) y^{\gamma-2} d y-\varepsilon^{2} c_{s}^{2} \rho_{1} \\
& =c_{s}^{2} \xi+\mathcal{R},
\end{aligned}
$$

where

$$
\mathcal{R}=\mathcal{R}(\zeta):=\gamma(\gamma-1) \int_{\rho_{0}}^{\rho}(\rho-y) y^{\gamma-2} d y \leqq C \zeta^{2} \leqq C\left(\varepsilon^{4} \rho_{1}^{2}+\xi^{2}\right),
$$

with $C=C\left(\left\|\rho^{\gamma-2}\right\|_{L^{\infty}},\left\|\rho_{0}^{\gamma-2}\right\|_{L^{\infty}}\right)$. Therefore, by denoting

$$
\begin{aligned}
& Q_{p}:=\rho_{0}^{-1}\left(\nabla_{h}\left(c_{s}^{2} \rho_{1}\right)-\mu \Delta_{h} v_{p}-\lambda \nabla_{h} \operatorname{div}_{h} v_{p}-\partial_{z z} v_{p}\right), \\
& \mathcal{F}_{1}:=\zeta Q_{p}, \\
& \mathcal{F}_{2}:=-\rho \psi^{h} \cdot \nabla_{h} v_{p}-\rho \psi^{z} \partial_{z} v_{p}, \\
& \mathcal{G}_{1}:=-\left(\operatorname{div}_{h}(\xi v)+\partial_{z}(\xi w)\right), \\
& \mathcal{G}_{2}:=-\varepsilon^{2}\left(\partial_{t} \rho_{1}+\operatorname{div}_{h}\left(\rho_{1} v\right)+\partial_{z}\left(\rho_{1} w\right)\right),
\end{aligned}
$$

we can write system (1.4) as

$$
\begin{cases}\partial_{t} \xi+\rho_{0}\left(\operatorname{div}_{h} \psi^{h}+\partial_{z} \psi^{z}\right)=\mathcal{G}_{1}+\mathcal{G}_{2} & \text { in } \Omega_{h} \times 2 \mathbb{T}, \\ \rho \partial_{t} \psi^{h}+\rho v \cdot \nabla_{h} \psi^{h}+\rho w \partial_{z} \psi^{h}+\nabla_{h}\left(\varepsilon^{-2} c_{s}^{2} \xi\right)=\mu \Delta_{h} \psi^{h} & \\ \quad+\lambda \nabla_{h} \operatorname{div}_{h} \psi^{h}+\partial_{z z} \psi^{h}+\mathcal{F}_{1}+\mathcal{F}_{2}-\nabla_{h}\left(\varepsilon^{-2} \mathcal{R}\right) & \text { in } \Omega_{h} \times 2 \mathbb{T}, \\ \partial_{z} \xi=0 & \text { in } \Omega_{h} \times 2 \mathbb{T} .\end{cases}
$$


In order to recover the vertical velocity perturbation $\psi^{z}$, we introduce the following notations, for any function $f$ in $\Omega_{h} \times 2 \mathbb{T}$, which is also even in the $z$-variable:

$$
\bar{f}(x, y, t):=\int_{0}^{1} f\left(x, y, z^{\prime}, t\right) \mathrm{d} z^{\prime} \text { and } \tilde{f}:=f-\bar{f} .
$$

The periodicity and symmetry of $f$ imply that $\bar{f}(x, y, t)=\int_{k}^{k+1} f\left(x, y, z^{\prime}, t\right) \mathrm{d} z^{\prime}$ for any $k \in \mathbb{Z}$. Notice that from $(\mathrm{CPE})_{3}, \rho$ is independent of the vertical variable $z$. Then by averaging $(\mathrm{CPE})_{1}$ over the vertical direction, thanks to (1.5), one will get

$$
\begin{aligned}
\partial_{t} \rho+\operatorname{div}_{h}(\rho \bar{v})= & 0, \text { and after comparing with }(C P E)_{1}, \\
& \operatorname{div}_{h}(\rho \widetilde{v})+\partial_{z}(\rho w)=0 .
\end{aligned}
$$

In particular, from the above, the vertical velocity $w$ is determined through $\rho, v$ by the formula, thanks to (1.5):

$$
\begin{aligned}
\rho w= & -\int_{0}^{z}\left(\rho \operatorname{div}_{h} v_{p}+\rho \operatorname{div}_{h} \psi^{h}-\rho \operatorname{div}_{h} \overline{\psi^{h}}+v \cdot \nabla_{h} \rho\right. \\
& \left.-\bar{v} \cdot \nabla_{h} \rho\right) \mathrm{d} z^{\prime}, \quad \text { and therefore } \\
\rho \psi^{z}= & -\int_{0}^{z}\left(\operatorname{div}_{h}\left(\rho \widetilde{\psi^{h}}\right)+\widetilde{v_{p}} \cdot \nabla_{h} \rho\right) \mathrm{d} z^{\prime},
\end{aligned}
$$

where we have substituted the following identity thanks to $(\mathrm{PE})_{1}$ and (1.5),

$$
w_{p}=-\int_{0}^{z} \operatorname{div}_{h} v_{p} \mathrm{~d} z^{\prime} .
$$

Such facts imply that in (CPE), (PE) and (1.8), the vertical velocities and the vertical perturbation, i.e., $w^{\varepsilon}, w_{p}, \psi^{\varepsilon, z}$, are fully determined by $v^{\varepsilon}, v_{p}, \rho^{\varepsilon}$. Therefore, there is no need to impose initial data for $w^{\varepsilon}, w_{p}, \psi^{\varepsilon, z}$.

System (1.8) (or equivalently (1.4)) is complemented with initial data,

$$
\begin{aligned}
\left.\left(\xi, \psi^{h}\right)\right|_{t=0}= & \left(\xi_{i n}, \psi_{i n}^{h}\right) \in H^{2}\left(\Omega_{h} \times 2 \mathbb{T} ; \mathbb{R}\right) \times H^{2}\left(\Omega_{h} \times 2 \mathbb{T} ; \mathbb{R}^{2}\right), \text { where } \\
& \partial_{z} \xi_{\text {in }}=0, \text { and } \psi_{i n}^{h} \text { is even in the } z \text {-variable, }
\end{aligned}
$$

with the compatibility conditions

$$
\begin{aligned}
& \xi_{i n, 1}=-\rho_{0}\left(\operatorname{div}_{h} \psi_{i n}^{h}+\partial_{z} \psi_{i n}^{z}\right)+\mathcal{G}_{1, i n}+\mathcal{G}_{2, i n} \quad \text { in } \Omega_{h} \times 2 \mathbb{T}, \\
& \rho_{i n}^{\varepsilon} \psi_{i n, 1}^{h}+\rho_{i n}^{\varepsilon} v_{i n}^{\varepsilon} \cdot \nabla_{h} \psi_{i n}^{h}+\rho_{i n}^{\varepsilon} w_{i n}^{\varepsilon} \partial_{z} \psi_{i n}^{h}+\nabla\left(\varepsilon^{-2} c_{s}^{2} \xi\right)=\mu \Delta_{h} \psi_{i n}^{h} \\
& \quad+\lambda \nabla_{h} \operatorname{div}_{h} \psi_{i n}^{h}+\partial_{z z} \psi_{i n}^{h}+\mathcal{F}_{1, i n}+\mathcal{F}_{2, i n}-\nabla_{h}\left(\varepsilon^{-2} \mathcal{R}\right) \quad \text { in } \Omega_{h} \times 2 \mathbb{T}, \\
& \quad \partial_{z} \xi_{i n}=0 \text { and } \partial_{z} \xi_{i n, 1}=0 \quad \text { in } \Omega_{h} \times 2 \mathbb{T}, \\
& \text { with }\left(\xi_{i n, 1}, \psi_{i n, 1}^{h}\right) \in L^{2}\left(\Omega_{h} \times 2 \mathbb{T}\right) \times L^{2}\left(\Omega_{h} \times 2 \mathbb{T}\right),
\end{aligned}
$$

where $\rho_{i n}^{\varepsilon}=\rho_{0}+\varepsilon^{2} \rho_{1, i n}+\xi_{i n}, v_{i n}^{\varepsilon}=v_{p, i n}+\psi_{i n}^{h}, w_{i n}^{\varepsilon}=w_{p, i n}+\psi_{i n}^{z}$, and $\psi_{i n}^{z}$ is given by

$$
\rho_{i n}^{\varepsilon} \psi_{i n}^{z}=-\int_{0}^{z}\left(\operatorname{div}_{h}\left(\rho_{i n}^{\varepsilon} \widetilde{\psi_{i n}^{h}}\right)+\widetilde{v}_{p, i n} \cdot \nabla_{h} \rho_{i n}^{\varepsilon}\right) \mathrm{d} z^{\prime}
$$


Here $v_{p, \text { in }}, \rho_{1, \text { in }}, \rho_{1, i n, 1}, w_{p, \text { in }}, \mathcal{G}_{i, \text { in }}, \mathcal{F}_{i, \text { in }}$ are initial values of $v_{p}, \rho_{1}, \partial_{t} \rho_{1}, w_{p}$, $\mathcal{G}_{i}, \mathcal{F}_{i}, i \in\{1,2\}$, respectively, while $w_{p, \text { in }}$ is given by $w_{p, \text { in }}=-\int_{0}^{z} \operatorname{div}_{h} v_{p, \text { in }} \mathrm{d} z^{\prime}$.

It is worth stressing that we will choose the initial time derivatives of the perturbations, i.e., $\left(\varepsilon^{-1} \xi_{i n, 1}, \psi_{i n, 1}^{h}\right)$ in (1.13), to be bounded, uniformly in $\varepsilon$ [see (1.14) and Theorem 1.2]. The reason for such choices of initial data is to exclude the high-frequency acoustic waves which corresponds to the fact that our initial data are well-prepared.

We denote the initial energy functional by

$$
\mathcal{E}_{i n}:=\left\|\psi_{i n}^{h}\right\|_{H^{2}}^{2}+\left\|\varepsilon \psi_{i n, 1}^{h}\right\|_{L^{2}}^{2}+\left\|\varepsilon^{-1} \xi_{i n}\right\|_{H^{2}}^{2}+\left\|\xi_{i n, 1}\right\|_{L^{2}}^{2} .
$$

Remark 1. $\left(\rho_{i n}^{\varepsilon}, v_{i n}^{\varepsilon}\right)$ is the corresponding initial datum of $\left(\rho^{\varepsilon}, v^{\varepsilon}\right)$ for system (CPE). $v_{p, i n}$ is the initial datum of $v_{p}$ for system (PE). Accordingly, $\rho_{1, i n}=$ $\rho_{1, \text { in }}(x, y), \rho_{1, \text { in }, 1}=\rho_{1, \text { in }, 1}(x, y)$ are determined by the following elliptic problems:

$$
\begin{aligned}
& \left\{\begin{array}{l}
-c_{s}^{2} \Delta_{h} \rho_{1, i n}=\rho_{0} \int_{0}^{1} \operatorname{div}_{h}\left(\operatorname{div}_{h}\left(v_{p, i n} \otimes v_{p, i n}\right)\right) \mathrm{d} z \text { in } \Omega_{h}, \\
\int_{\Omega_{h}} \rho_{1, i n} \mathrm{~d} x \mathrm{~d} y=0
\end{array}\right. \\
& \left\{\begin{array}{l}
-c_{s}^{2} \Delta_{h} \rho_{1, i n, 1}=2 \rho_{0} \int_{0}^{1} \operatorname{div}_{h}\left(\operatorname{div}_{h}\left(v_{p, i n} \otimes v_{p, i n, 1}\right)\right) \mathrm{d} z \\
\operatorname{in} \Omega_{h}, \quad \int_{\Omega_{h}} \rho_{1, i n, 1} \mathrm{~d} x \mathrm{~d} y=0,
\end{array}\right.
\end{aligned}
$$

where $v_{p, i n, 1}$ is the initial value of $\partial_{t} v_{p}$ determined by

$$
\begin{aligned}
\rho_{0} v_{p, i n, 1}= & -\rho_{0}\left(v_{p, i n} \cdot \nabla_{h} v_{p, i n}+w_{p, i n} \partial_{z} v_{p, i n}\right)-\nabla_{h}\left(c_{s}^{2} \rho_{1, i n}\right) \\
& +\mu \Delta_{h} v_{p, i n}+\lambda \nabla_{h} \operatorname{div}_{h} v_{p, i n}+\partial_{z z} v_{p, i n} \quad \text { in } \Omega_{h} \times 2 \mathbb{T} .
\end{aligned}
$$

As we stated before, we focus in this work on the asymptotic limit as $\varepsilon \rightarrow 0^{+}$. We have the following global regularity of the limit system (PE):

Theorem 1.1. (Global regularity of the PE) For $\lambda<4 \mu<12 \lambda$, suppose that (PE) is complemented with initial data $v_{p, i n} \in H^{1}\left(\Omega_{h} \times 2 \mathbb{T}\right)$, which is even in the $z$-variable, and satisfies the compatibility conditions

$$
\int_{\Omega_{h} \times 2 \mathbb{T}} v_{p, i n} \mathrm{~d} \vec{x}=0, \operatorname{div}_{h} \bar{v}_{p, i n}=0 .
$$

Then there exists a solution $\left(v_{p}, \rho_{1}\right)$, with $\int_{0}^{1} \operatorname{div}_{h} v_{p} \mathrm{~d} z=0$ and $w_{p}$ given by (1.11), to the primitive equations (PE). Moreover, there is a constant $C_{p, i n}$ depending only $\left\|v_{p, i n}\right\|_{H^{1}}$ such that

$$
\begin{aligned}
& \sup _{0 \leqq t<\infty}\left(\left\|v_{p}(t)\right\|_{H^{1}}^{2}+\left\|\partial_{t} v_{p}\right\|_{H^{-1}}^{2}\right)+\int_{0}^{\infty}\left(\left\|\nabla v_{p}(t)\right\|_{H^{1}}^{2}\right. \\
& \left.\quad+\left\|\partial_{t} v_{p}(t)\right\|_{L^{2}}^{2}\right) \mathrm{d} t \leqq C_{p, i n} .
\end{aligned}
$$


Moreover,

$$
\left\|v_{p}(t)\right\|_{L^{2}}^{2} \leqq C e^{-c t}\left\|v_{p, i n}\right\|_{L^{2}}^{2}
$$

for some positive constants $c, C$. In addition, if $v_{p, i n} \in H^{s}\left(\Omega_{h} \times 2 \mathbb{T}\right)$ for any integer $s \geqq 2$, there is a positive constant $C_{p, i n, s}$, depending only on $\left\|v_{p, \text { in }}\right\|_{H^{s}}$ such that

$$
\begin{aligned}
& \sup _{0 \leqq t<\infty}\left(\left\|v_{p}(t)\right\|_{H^{s}}^{2}+\left\|\partial_{t} v_{p}(t)\right\|_{H^{s-2}}^{2}\right)+\int_{0}^{\infty}\left(\left\|v_{p}(t)\right\|_{H^{s+1}}^{2}\right. \\
& \left.\quad+\left\|\partial_{t} v_{p}(t)\right\|_{H^{s-1}}^{2}\right) \mathrm{d} t \leqq C_{p, i n, s} .
\end{aligned}
$$

Proof. The local well-posedness of solutions to (PE) in the function space $H^{s}$ has been established in [56]. What is left is the global regularity estimate, which is a direct consequence of Proposition 3.

Remark 2. We only have to be careful about the different estimates caused by the viscosity tensor. In particular, it is the $L^{q}$ estimate of $v_{p}$, below in Section 5 , that requires the constraints on the viscosity coefficients. For solutions with $H^{2}$ initial data, the result can be found in [41]. The new thing we treat here is the case when $v_{p, i n} \in H^{s}$, for $s>2$.

Remark 3. The regularity of $\rho_{1}$ can be obtained by solving elliptic problem (4.1).

Now we can state our main theorem in this work.

Theorem 1.2. (Low Mach number limit of the CPE) For $\lambda<4 \mu<12 \lambda$, suppose $v_{p, i n} \in H^{s}\left(\Omega_{h} \times 2 \mathbb{T}\right)$, with integer $s \geqq 3$, and it satisfies the compatibility conditions (1.15). Also, we complement (1.8) with initial data $\left.\left(\xi^{\varepsilon}, \psi^{\varepsilon, h}\right)\right|_{t=0}=$ $\left(\xi_{\text {in }}, \psi_{i n}^{h}\right) \in H^{2}\left(\Omega_{h} \times 2 \mathbb{T}\right) \times H^{2}\left(\Omega_{h} \times 2 \mathbb{T}\right)$ as in (1.12), which satisfies the compatibility conditions (1.13). Recall that we also require $v_{p, i n}, \psi_{i n}^{h}$ to be even in the $z$-variable. Then there exists a positive constant $\varepsilon_{0} \in(0,1)$ small enough, such that if $\varepsilon \in\left(0, \varepsilon_{0}\right)$ and $\mathcal{E}_{i n} \leqq \varepsilon^{2}$, there exists a global unique strong solution $\left(\xi^{\varepsilon}, \psi^{\varepsilon, h}\right)$ to system (1.8). In particular, the following regularity is satisfied:

$$
\begin{aligned}
& \xi^{\varepsilon} \in L^{\infty}\left(0, \infty ; H^{2}\left(\Omega_{h} \times 2 \mathbb{T}\right)\right), \\
& \partial_{t} \xi^{\varepsilon} \in L^{\infty}\left(0, \infty ; L^{2}\left(\Omega_{h} \times 2 \mathbb{T}\right)\right) \cap L^{2}\left(0, \infty ; L^{2}\left(\Omega_{h} \times 2 \mathbb{T}\right)\right), \\
& \nabla_{h} \xi^{\varepsilon} \in L^{2}\left(0, \infty ; H^{1}\left(\Omega_{h} \times 2 \mathbb{T}\right)\right), \psi^{\varepsilon, h} \in L^{\infty}\left(0, \infty ; H^{2}\left(\Omega_{h} \times 2 \mathbb{T}\right)\right), \\
& \partial_{t} \psi^{\varepsilon, h} \in L^{\infty}\left(0, \infty ; L^{2}\left(\Omega_{h} \times 2 \mathbb{T}\right)\right) \cap L^{2}\left(0, \infty ; H^{1}\left(\Omega_{h} \times 2 \mathbb{T}\right)\right), \\
& \nabla \psi^{\varepsilon, h} \in L^{2}\left(0, \infty ; H^{2}\left(\Omega_{h} \times 2 \mathbb{T}\right)\right) .
\end{aligned}
$$

In addition, we have the following estimate:

$$
\begin{aligned}
& \sup _{0 \leqq}\{t<\infty \\
& \left.\quad+\left\|\psi_{t} \xi^{\varepsilon, h}(t)\right\|_{H^{2}}^{2}+\left\|\varepsilon \partial_{t} \psi^{\varepsilon, h}(t)\right\|_{L^{2}}^{2}\right\}+\| \int_{0}^{\infty}\left\{\left\|\nabla \psi^{\varepsilon, h}(t)\right\|_{H^{2}}^{2}+\left\|\varepsilon \partial_{t} \psi^{\varepsilon, h}(t)\right\|_{H^{2}}^{2} \|_{H^{1}}^{2}\right. \\
& \left.\quad+\left\|\varepsilon^{-1} \nabla_{h} \xi^{\varepsilon}(t)\right\|_{H^{1}}^{2}+\left\|\partial_{t} \xi^{\varepsilon}(t)\right\|_{L^{2}}^{2}\right\} \mathrm{d} t \leqq C \varepsilon^{2},
\end{aligned}
$$


for some positive constant $C$ depending only on $\left\|v_{p, \text { in }}\right\|_{H^{3}}$, which is independent of $\varepsilon$. In particular, $\left(\rho^{\varepsilon}, v^{\varepsilon}, w^{\varepsilon}\right)$ as in (1.3) is a globally defined strong solution to (CPE) and the following asymptotic estimate holds:

$$
\begin{aligned}
& \sup _{0 \leqq t<\infty}\left\{\left\|v^{\varepsilon}(t)-v_{p}(t)\right\|_{H^{2}}^{2}+\left\|\rho^{\varepsilon}(t)-\rho_{0}\right\|_{H^{2}}^{2}\right. \\
& \left.+\left\|w^{\varepsilon}(t)-w_{p}(t)\right\|_{H^{1}}^{2}\right\} \leqq C \varepsilon^{2},
\end{aligned}
$$

for some positive constant $C$ depending only on $\left\|v_{p, i n}\right\|_{H^{3}}$, which is independent of $\varepsilon$, where $w^{\varepsilon}, w_{p}$ are given as in (1.9), (1.11), respectively.

Remark 4. According to (1.18), the time derivatives, in comparison to the spatial derivatives, have larger perturbations. However, thanks to the well-prepared data setting, they are bounded.

This work will be organized as follows: in Section 2, we summarize the notations which will be commonly used in later paragraphs. Section 3 focuses on the $\varepsilon$ independent a priori estimates, which are the foundation of the low Mach number limit. In Section 4, we focus on the proof of Theorem 1.2. This will be shown through a continuity argument. Finally in Section 5, we summarize the proof of Theorem 1.1.

\section{Preliminaries}

We use the notations

$$
\int \cdot \mathrm{d} \vec{x}=\int_{\Omega_{h} \times 2 \mathbb{T}} \cdot \mathrm{d} \vec{x}:=\int_{\Omega_{h} \times 2 \mathbb{T}} \cdot d x d y d z, \int_{\Omega_{h}} \cdot \mathrm{d} x \mathrm{~d} y
$$

to represent the integrals in $\Omega$ and $\Omega_{h}$ respectively. Hereafter, $\partial_{h} \in\left\{\partial_{x}, \partial_{y}\right\}$ represents the horizontal derivatives, and $\partial_{z}$ represents the vertical derivative.

We will use $|\cdot|,\|\cdot\|$ to denote norms in $\Omega_{h} \subset \mathbb{R}^{2}$ and $\Omega_{h} \times 2 \mathbb{T} \subset \mathbb{R}^{3}$, respectively. After applying Ladyzhenskaya's and Agmon's inequalities in $\Omega_{h}$ and $\Omega$, directly we have

$$
\begin{aligned}
|f|_{L^{4}} & \leqq C|f|_{L^{2}}^{1 / 2}\left|\nabla_{h} f\right|_{L^{2}}^{1 / 2}+|f|_{L^{2}},|f|_{\infty} \leqq C|f|_{L^{2}}^{1 / 2}|f|_{H^{2}}^{1 / 2}, \\
\|f\|_{L^{3}} & \leqq C\|f\|_{L^{2}}^{1 / 2}\|\nabla f\|_{L^{2}}^{1 / 2}+\|f\|_{L^{2}}
\end{aligned}
$$

for the function $f$ with bounded right-hand sides. Also, applying Minkowski's and Hölder's inequalities yields

$$
\begin{aligned}
& |\bar{f}|_{L^{q}} \leqq \int_{0}^{1}|f(z)|_{L^{q}} \mathrm{~d} z \leqq C\|f\|_{L^{q}}, \\
& \text { and hence }\|\tilde{f}\|_{L^{q}} \leqq C\|f\|_{L^{q}}, q \in[1, \infty) .
\end{aligned}
$$


We use $\delta>0$ to denote a arbitrary constant which will be chosen later adaptively small. Correspondingly, $C_{\delta}$ is some positive constant depending on $\delta$. In addition, for any quantities $A$ and $B, A \lesssim B$ is used to denote that there exists a positive constant independent of the solutions such that $A \leqq C B$.

The following energy and dissipation functionals will be employed:

$$
\begin{aligned}
\mathcal{E}(t) & :=\left\|\psi^{h}(t)\right\|_{H^{2}}^{2}+\left\|\varepsilon \psi_{t}^{h}(t)\right\|_{L^{2}}^{2}+\left\|\varepsilon^{-1} \xi(t)\right\|_{H^{2}}^{2}+\left\|\xi_{t}(t)\right\|_{L^{2}}^{2}, \\
\mathcal{D}(t) & :=\left\|\nabla \psi^{h}(t)\right\|_{H^{2}}^{2}+\left\|\varepsilon \psi_{t}^{h}(t)\right\|_{H^{1}}^{2}+\left\|\varepsilon^{-1} \nabla_{h} \xi(t)\right\|_{H^{1}}^{2} \\
& +\left\|\xi_{t}(t)\right\|_{L^{2}}^{2} .
\end{aligned}
$$

Then $\mathcal{E}(0)=\mathcal{E}_{\text {in }}$, where $\mathcal{E}_{\text {in }}$ is as in (1.14). In this work, we shall use $Q(\mathcal{E})$ to denote a polynomial quantity, with positive coefficients, of $\sqrt{\mathcal{E}}$ and $Q(0)=0$. In general, $Q(\cdot)$ is a generic polynomial quantity, with positive coefficients, of the arguments and $Q(0)=0$.

\section{3. $\varepsilon$-Independent A Priori Estimate}

This section is devoted to show the following:

Proposition 1. For any $T>0, t \in[0, T]$, suppose that the solution $\left(v_{p}, \rho_{1}\right)$ with $w_{p}$ given by (1.11) to $(\mathrm{PE})$ satisfies

$$
\begin{aligned}
& \left\|v_{p}(t)\right\|_{H^{3}},\left\|v_{p, t}(t)\right\|_{L^{2}},\left\|\rho_{1}(t)\right\|_{H^{2}},\left\|\rho_{1, t}(t)\right\|_{L^{2}},\left\|w_{p}(t)\right\|_{H^{1}} \leqq C, \\
& \int_{0}^{T}\left(\left\|v_{p}(t)\right\|_{H^{3}}^{2}+\left\|v_{p, t}(t)\right\|_{H^{1}}^{2}+\left\|\rho_{1, t t}(t)\right\|_{L^{2}}^{2}+\left\|\rho_{1, t}(t)\right\|_{H^{1}}^{2}\right. \\
& \left.\quad+\left\|\rho_{1}(t)\right\|_{H^{2}}^{2}+\left\|w_{p}(t)\right\|_{H^{2}}^{2}+\left\|v_{p}(t)\right\|_{H^{2}}\right) \mathrm{d} t \leqq C
\end{aligned}
$$

for some positive constant $C$, and

$$
\frac{1}{2} \rho_{0}<\rho<2 \rho_{0} \quad \text { in }\left(\Omega_{h} \times 2 \mathbb{T}\right) \times[0, T) .
$$

Then any solution $\left(\psi^{h}, \xi\right)$ to (1.8), with initial data as in (1.12), provided that it exists in the time interval $[0, T]$, with $\psi^{z}$ given by (1.10), satisfies

$$
\begin{aligned}
\sup _{0 \leqq t \leqq T} \mathcal{E}(t)+\int_{0}^{T} \mathcal{D}(t) \mathrm{d} t \leqq C^{\prime} e^{C^{\prime}+Q\left(\sup _{0 \leqq t \leqq T} \mathcal{E}(t)\right)}\left\{\varepsilon^{2}+\mathcal{E}_{i n}\right. \\
\left.\quad+\left(\varepsilon^{2}+\left(\varepsilon^{2}+1\right) Q\left(\sup _{0 \leqq t \leqq T} \mathcal{E}(t)\right)\right) \int_{0}^{T} \mathcal{D}(t) \mathrm{d} t\right\}
\end{aligned}
$$

for some positive constant $C^{\prime}$ depending only on the bounds in (3.1). In particular, $C^{\prime}$ is independent of $\varepsilon$ and $T$. 
Remark 5. We remark here that, from the definition of $\mathcal{E}(t)$ in (2.2) and (1.3), (3.2) automatically holds for $\varepsilon$ small enough if $\sup _{0 \leqq t \leqq T} \mathcal{E}(t)<\infty$ and (3.1) holds.

Throughout the rest of this section, it is assumed that $\left(\xi, \psi^{h}\right)$ with $\psi^{z}$ given by (1.10) is a solution to (1.8) which is smooth enough such that the estimates below can be established. To justify the arguments, one can employ the local wellposedness theory and the standard different quotient method to the corresponding lines below (replaced the differential operators by different quotients, for example); see, for instance, similar arguments in $[49,50]$.

We denote by $\mathfrak{G}_{p}(t)$ a polynomial, with positive coefficients, quantity of the arguments

$$
\left\|v_{p}(t)\right\|_{H^{3}},\left\|v_{p, t}(t)\right\|_{L^{2}},\left\|\rho_{1}(t)\right\|_{H^{2}},\left\|\rho_{1, t}(t)\right\|_{L^{2}},\left\|w_{p}(t)\right\|_{H^{1}}
$$

and

$$
\begin{aligned}
\mathfrak{H}_{p}(t): & =\left\|v_{p}(t)\right\|_{H^{3}}^{2}+\left\|v_{p, t}(t)\right\|_{H^{1}}^{2}+\left\|\rho_{1, t t}(t)\right\|_{L^{2}}^{2}+\left\|\rho_{1, t}(t)\right\|_{H^{1}}^{2} \\
& +\left\|\rho_{1}(t)\right\|_{H^{2}}^{2}+\left\|w_{p}(t)\right\|_{H^{2}}^{2}+\left\|v_{p}(t)\right\|_{H^{2}} .
\end{aligned}
$$

In particular, (3.1) of Proposition 1 is equivalent to

$$
\sup _{0 \leqq t \leqq T} \mathfrak{G}_{p}(t)+\int_{0}^{T} \mathfrak{H}_{p}(t) \mathrm{d} t<C
$$

for some positive constant $C$. For the sake of convenience, we will shorten the notations $\mathfrak{G}_{p}=\mathfrak{G}_{p}(t), \mathfrak{H}_{p}=\mathfrak{H}_{p}(t)$, below. We also remind the reader that we have assumed that (3.1) and (3.2) hold throughout this section.

\subsection{Temporal Derivatives}

We start by performing the time derivative estimate to the solutions to system (1.8). Applying $\partial_{t}$ to system (1.8) we will have the following system:

$$
\begin{cases}\partial_{t} \xi_{t}+\rho_{0}\left(\operatorname{div}_{h} \psi_{t}^{h}+\partial_{z} \psi_{t}^{z}\right)=\mathcal{G}_{1, t}+\mathcal{G}_{2, t} & \text { in } \Omega_{h} \times 2 \mathbb{T}, \\ \rho \partial_{t} \psi_{t}^{h}+\rho v \cdot \nabla_{h} \psi_{t}^{h}+\rho w \partial_{z} \psi_{t}^{h}+\nabla_{h}\left(\varepsilon^{-2} c_{s}^{2} \xi_{t}\right)=\mu \Delta_{h} \psi_{t}^{h} & \\ \quad+\lambda \nabla_{h} \operatorname{div}_{h} \psi_{t}^{h}+\partial_{z z} \psi_{t}^{h}+\mathcal{F}_{1, t}+\mathcal{F}_{2, t}-\nabla_{h}\left(\varepsilon^{-2} \mathcal{R}_{t}\right)+\mathcal{H}_{t} & \text { in } \Omega_{h} \times 2 \mathbb{T},\end{cases}
$$

where

$$
\mathcal{H}_{t}:=-\rho_{t} \psi_{t}^{h}-\left(\rho_{t} v+\rho v_{t}\right) \cdot \nabla_{h} \psi^{h}-\left(\rho_{t} w+\rho w_{t}\right) \partial_{z} \psi^{h} .
$$

We will show the following:

Lemma 1. In addition to the assumptions in Proposition 1, suppose that $\left(\xi, \psi^{h}\right)$, with $\psi^{z}$ given by (1.10), is a smooth solution to (1.8) in the time interval $[0, T]$. 
We have

$$
\begin{aligned}
& \frac{d}{\mathrm{~d} t}\left\{\frac{1}{2}\left\|\rho^{1 / 2} \varepsilon \psi_{t}^{h}\right\|_{L^{2}}^{2}+\frac{c_{s}^{2}}{2 \rho_{0}}\left\|\xi_{t}\right\|_{L^{2}}^{2}\right\}+\mu\left\|\varepsilon \nabla_{h} \psi_{t}^{h}\right\|_{L^{2}}^{2} \\
& \quad+\lambda\left\|\varepsilon \operatorname{div}_{h} \psi_{t}^{h}\right\|_{L^{2}}^{2}+\left\|\varepsilon \partial_{z} \psi_{t}^{h}\right\|_{L^{2}}^{2} \leqq \delta\left(\left\|\xi_{t}\right\|_{L^{2}}^{2}+\left\|\varepsilon \nabla \psi_{t}^{h}\right\|_{L^{2}}^{2}\right. \\
& \left.\quad+\left\|\varepsilon \psi_{t}^{h}\right\|_{L^{2}}^{2}+\left\|\nabla \psi^{h}\right\|_{H^{2}}^{2}\right)+\varepsilon^{2} C_{\delta}\left(Q(\mathcal{E})+\mathfrak{G}_{p}\right)\left\|\partial_{z} \psi^{h}\right\|_{H^{2}}^{2} \\
& \quad+C_{\delta} Q(\mathcal{E})\left(\left\|\varepsilon \psi_{t}^{h}\right\|_{L^{2}}^{2}+\left\|\nabla \psi^{h}\right\|_{H^{1}}^{2}+\left\|\xi_{t}\right\|_{L^{2}}^{2}\right) \\
& \quad+C_{\delta}\left(Q(\mathcal{E})+1+\mathfrak{G}_{p}\right) \mathfrak{H}_{p}\left(\left\|\psi^{h}\right\|_{H^{2}}^{2}+\left\|\varepsilon \psi_{t}^{h}\right\|_{L^{2}}^{2}+\varepsilon^{2}\right) .
\end{aligned}
$$

Proof. Take the $L^{2}$-inner product of (3.5) 2 with $\varepsilon^{2} \psi_{t}^{h}$. After applying integration by parts and substituting $(\mathrm{CPE})_{1}$ and $(3.5)_{1}$, we have the following:

$$
\begin{aligned}
& \frac{d}{\mathrm{~d} t}\left\{\frac{1}{2}\left\|\rho^{1 / 2} \varepsilon \psi_{t}^{h}\right\|_{L^{2}}^{2}+\frac{c_{s}^{2}}{2 \rho_{0}}\left\|\xi_{t}\right\|_{L^{2}}^{2}\right\}+\mu\left\|\varepsilon \nabla_{h} \psi_{t}^{h}\right\|_{L^{2}}^{2} \\
& \quad+\lambda\left\|\varepsilon \operatorname{div}_{h} \psi_{t}^{h}\right\|_{L^{2}}^{2}+\left\|\varepsilon \partial_{z} \psi_{t}^{h}\right\|_{L^{2}}^{2}=\int \varepsilon^{2} \mathcal{F}_{1, t} \cdot \psi_{t}^{h} \mathrm{~d} \vec{x} \\
& \quad+\int \varepsilon^{2} \mathcal{F}_{2, t} \cdot \psi_{t}^{h} \mathrm{~d} \vec{x}+\rho_{0}^{-1} c_{s}^{2} \int \xi_{t} \mathcal{G}_{1, t} \mathrm{~d} \vec{x}+\rho_{0}^{-1} c_{s}^{2} \int \xi_{t} \mathcal{G}_{2, t} \mathrm{~d} \vec{x} \\
& \quad+\int \varepsilon^{2} \mathcal{H}_{t} \cdot \psi_{t}^{h} \mathrm{~d} \vec{x}-\int \nabla_{h} \mathcal{R}_{t} \cdot \psi_{t}^{h} \mathrm{~d} \vec{x}=: \sum_{i=1}^{6} I_{i} .
\end{aligned}
$$

Next we estimate the right-hand side of (3.8). After substituting (3.6) into $I_{5}$, it can be written as

$$
\begin{aligned}
I_{5}= & -\int \zeta_{t}\left|\varepsilon \psi_{t}^{h}\right|^{2} \mathrm{~d} \vec{x}-\int\left(\varepsilon^{2} \zeta_{t} v \cdot \nabla_{h} \psi^{h} \cdot \psi_{t}^{h}+\varepsilon^{2} \rho v_{t} \cdot \nabla_{h} \psi^{h} \cdot \psi_{t}^{h}\right) \mathrm{d} \vec{x} \\
& -\int\left(\varepsilon^{2} \zeta_{t} w \partial_{z} \psi^{h} \cdot \psi_{t}^{h}+\varepsilon^{2} \rho w_{t} \partial_{z} \psi^{h} \cdot \psi_{t}^{h}\right) \mathrm{d} \vec{x}=: I_{5}^{\prime}+I_{5}^{\prime \prime}+I_{5}^{\prime \prime \prime} .
\end{aligned}
$$

Notice that $\zeta=\varepsilon^{2} \rho_{1}+\xi$ is independent of the $z$ variable. Then, for every $\delta>0$ there exists a positive constant $C_{\delta}$ such that

$$
\begin{aligned}
I_{5}^{\prime} & \lesssim\left\|\zeta_{t}\right\|_{L^{2}} \int_{0}^{1}\left|\varepsilon \psi_{t}^{h}\right|_{L^{4}}^{2} \mathrm{~d} z \lesssim\left\|\zeta_{t}\right\|_{L^{2}} \int_{0}^{1}\left(\left|\varepsilon \psi_{t}^{h}\right|_{L^{2}}\left|\varepsilon \nabla_{h} \psi_{t}^{h}\right|_{L^{2}}+\left|\varepsilon \psi_{t}^{h}\right|_{L^{2}}^{2}\right) \mathrm{d} z \\
& \lesssim\left\|\zeta_{t}\right\|_{L^{2}}\left(\left\|\varepsilon \psi_{t}^{h}\right\|_{L^{2}}\left\|\varepsilon \nabla_{h} \psi_{t}^{h}\right\|_{L^{2}}+\left\|\varepsilon \psi_{t}^{h}\right\|_{L^{2}}^{2}\right) \lesssim \delta\left\|\varepsilon \nabla_{h} \psi_{t}^{h}\right\|_{L^{2}}^{2} \\
& +\delta\left\|\varepsilon \psi_{t}^{h}\right\|_{L^{2}}^{2}+C_{\delta}\left(\varepsilon \varepsilon^{4}\left\|\rho_{1, t}\right\|_{L^{2}}^{2}+\mathcal{E}\right)\left\|\varepsilon \psi_{t}^{h}\right\|_{L^{2}}^{2},
\end{aligned}
$$

where we have applied the Minkowski, Hölder's, the Sobolev embedding and Young's inequalities. On the other hand, $I_{5}^{\prime \prime}$ can be estimated directly using Hölder's, the Sobolev embedding and Young's inequalities:

$$
\begin{aligned}
I_{5}^{\prime \prime} \lesssim & \delta\left\|\varepsilon \nabla \psi_{t}^{h}\right\|_{L^{2}}^{2}+\delta\left\|\varepsilon \psi_{t}^{h}\right\|_{L^{2}}^{2}+C_{\delta} Q(\mathcal{E})\left(\left\|\nabla_{h} \psi^{h}\right\|_{H^{1}}^{2}+\left\|\varepsilon \psi_{t}^{h}\right\|_{L^{2}}^{2}\right) \\
& +C_{\delta}\left(Q(\mathcal{E})+1+\mathfrak{G}_{p}\right) \mathfrak{H}_{p}\left(\left\|\psi^{h}\right\|_{H^{2}}^{2}+\left\|\varepsilon \psi_{t}^{h}\right\|_{L^{2}}^{2}\right) .
\end{aligned}
$$


On the other hand, from (1.9) (or (1.10)), we have the identities

$$
\begin{aligned}
w & =w_{p}-\int_{0}^{z}\left(\operatorname{div}_{h} \widetilde{\psi^{h}}+\widetilde{v} \cdot \nabla_{h} \log \rho\right) \mathrm{d} z^{\prime} \\
w_{t} & =w_{p, t}-\int_{0}^{z}\left(\operatorname{div}_{h} \widetilde{\psi_{t}^{h}}+\widetilde{v}_{t} \cdot \nabla_{h} \log \rho+\widetilde{v} \cdot \nabla_{h}(\log \rho)_{t}\right) \mathrm{d} z^{\prime} .
\end{aligned}
$$

Therefore, $I_{5}^{\prime \prime \prime}$ can be written as

$$
\begin{aligned}
I_{5}^{\prime \prime \prime}= & -\int \varepsilon^{2}\left(\zeta_{t} w_{p}+\rho w_{p, t}\right)\left(\partial_{z} \psi^{h} \cdot \psi_{t}^{h}\right) \mathrm{d} \vec{x}+\int\left[\varepsilon ^ { 2 } \int _ { 0 } ^ { z } \left(\operatorname{div}_{h} \widetilde{\psi^{h}}\right.\right. \\
& \left.\left.+\widetilde{v} \cdot \nabla_{h} \log \rho\right) \mathrm{d} z^{\prime} \times\left(\zeta_{t} \partial_{z} \psi^{h} \cdot \psi_{t}^{h}\right)\right] \mathrm{d} \vec{x}+\int\left[\varepsilon ^ { 2 } \int _ { 0 } ^ { z } \left(\rho \operatorname{div}_{h} \widetilde{\psi_{t}^{h}}\right.\right. \\
& \left.\left.+\widetilde{v}_{t} \cdot \nabla_{h} \rho+\rho \tilde{v} \cdot \nabla_{h}(\log \rho)_{t}\right) \mathrm{~d} z^{\prime} \times\left(\partial_{z} \psi^{h} \cdot \psi_{t}^{h}\right)\right] \mathrm{d} \vec{x}=: \sum_{i=1}^{3} I_{5, i}^{\prime \prime \prime}
\end{aligned}
$$

Then, we plug in identity (1.11) and apply the Hölder, Minkowski and Young inequalities to infer

$$
\begin{aligned}
I_{5,1}^{\prime \prime \prime} \lesssim & \varepsilon \int_{0}^{1}\left[\left(\int_{0}^{z}\left|\nabla_{h} v_{p}\right|_{L^{8}} \mathrm{~d} z^{\prime}\right) \times\left(\left|\zeta_{t}\right|_{L^{2}}\left|\partial_{z} \psi^{h}\right|_{L^{8}}\left|\varepsilon \psi_{t}^{h}\right|_{L^{4}}\right)\right] \mathrm{d} z \\
& +\varepsilon \int_{0}^{1}\left[\left(\int_{0}^{z}\left|v_{p, t}\right|_{L^{2}} \mathrm{~d} z^{\prime}\right) \times\left(\left|\nabla_{h} \zeta\right|_{L^{8}}\left|\partial_{z} \psi^{h}\right|_{L^{8}}\left|\varepsilon \psi_{t}^{h}\right|_{L^{4}}\right)\right] \mathrm{d} z \\
& +\varepsilon \int_{0}^{1}\left[\left(\int_{0}^{z}\left|v_{p, t}\right|_{L^{4}} \mathrm{~d} z^{\prime}\right) \times\left(|\rho|_{L^{\infty}}\left|\partial_{z} \nabla_{h} \psi^{h}\right|_{L^{2}}\left|\varepsilon \psi_{t}^{h}\right|_{L^{4}}\right.\right. \\
& \left.\left.+|\rho|_{L^{\infty}}\left|\partial_{z} \psi^{h}\right|_{L^{4}}\left|\varepsilon \nabla_{h} \psi_{t}^{h}\right|_{L^{2}}\right)\right] \mathrm{d} z \lesssim \delta\left\|\varepsilon \nabla \psi_{t}^{h}\right\|_{L^{2}}^{2}+\delta\left\|\varepsilon \psi_{t}^{h}\right\|_{L^{2}}^{2} \\
& +C_{\delta}\left(Q(\mathcal{E})+1+\mathfrak{G}_{p}\right) \mathfrak{H}_{p}\left\|\psi^{h}\right\|_{H^{2}}^{2} .
\end{aligned}
$$

On the other hand, a straight forward estimate shows that

$$
\begin{aligned}
I_{5,2}^{\prime \prime \prime} & \lesssim \varepsilon \int_{0}^{1}\left(\left|\nabla_{h} \psi^{h}\right|_{L^{8}}+|v|_{L^{\infty}}\left|\nabla_{h} \zeta\right|_{L^{8}}\right) \mathrm{d} z \times \int_{0}^{1}\left|\zeta_{t}\right|_{L^{2}}\left|\partial_{z} \psi^{h}\right|_{L^{8}}\left|\varepsilon \psi_{t}^{h}\right|_{L^{4}} \mathrm{~d} z \\
& \lesssim \delta\left\|\varepsilon \nabla \psi_{t}^{h}\right\|_{L^{2}}^{2}+\delta\left\|\varepsilon \psi_{t}^{h}\right\|_{L^{2}}^{2}+C_{\delta} Q(\mathcal{E})\left\|\partial_{z} \psi^{h}\right\|_{H^{1}}^{2} \\
& +C_{\delta}\left(Q(\mathcal{E})+1+\mathfrak{G}_{p}\right) \mathfrak{H}_{p}\left\|\psi^{h}\right\|_{H^{2}}^{2} .
\end{aligned}
$$

To estimate $I_{5,3}^{\prime \prime \prime}$, we apply integration by parts as follows:

$$
\begin{aligned}
I_{5,3}^{\prime \prime \prime}= & \int\left[\varepsilon^{2} \int_{0}^{z}\left(\rho \operatorname{div}_{h} \widetilde{\psi_{t}^{h}}+\widetilde{v}_{t} \cdot \nabla_{h} \rho-\zeta_{t} \widetilde{v} \cdot \nabla_{h} \log \rho-\zeta_{t} \operatorname{div}_{h} \widetilde{v}\right) \mathrm{d} z\right. \\
& \left.\times\left(\partial_{z} \psi^{h} \cdot \psi_{t}^{h}\right)\right] \mathrm{d} \vec{x}-\int\left[\varepsilon^{2} \int_{0}^{z} \zeta_{t} \widetilde{v} \mathrm{~d} z \cdot \nabla_{h}\left(\partial_{z} \psi^{h} \cdot \psi_{t}^{h}\right)\right] \mathrm{d} \vec{x},
\end{aligned}
$$


from which we infer

$$
\begin{aligned}
I_{5,3}^{\prime \prime \prime} \lesssim & \int_{0}^{1}\left(|\rho|_{L^{\infty}}\left|\varepsilon \nabla_{h} \psi_{t}^{h}\right|_{L^{2}}+\left|\varepsilon v_{t}\right|_{L^{4}}\left|\nabla_{h} \zeta\right|_{L^{4}}\right) \mathrm{d} z \\
& \times \int_{0}^{1}\left|\partial_{z} \psi^{h}\right|_{L^{4}}\left|\varepsilon \psi_{t}^{h}\right|_{L^{4}} \mathrm{~d} z+\int_{0}^{1}\left|\partial_{z} \psi^{h}\right|_{L^{8}}\left|\varepsilon \psi_{t}^{h}\right|_{L^{4}} \mathrm{~d} z \\
& \times \int_{0}^{1}\left(\varepsilon\left|\zeta_{t}\right|_{L^{2}}|v|_{\infty}\left|\nabla_{h} \zeta\right|_{L^{8}}+\varepsilon\left|\zeta_{t}\right|_{L^{2}}\left|\nabla_{h} v\right|_{L^{8}}\right) \mathrm{d} z \\
& +\int_{0}^{1}\left(\left|\partial_{z} \psi^{h}\right|_{L^{\infty}}\left|\varepsilon \nabla_{h} \psi_{t}^{h}\right|_{L^{2}}+\left|\nabla_{h} \partial_{z} \psi^{h}\right|_{L^{4}}\left|\varepsilon \psi_{t}^{h}\right|_{L^{4}}\right) \mathrm{d} z \\
& \times \int_{0}^{1} \varepsilon\left|\zeta_{t}\right|_{L^{2}}|v|_{L^{\infty}} \mathrm{d} z \\
\lesssim & \delta\left\|\varepsilon \nabla_{h} \psi_{t}^{h}\right\|_{L^{2}}^{2}+\delta\left\|\varepsilon \psi_{t}^{h}\right\|_{L^{2}}^{2}+\varepsilon^{2} C_{\delta}\left(Q(\mathcal{E})+\mathfrak{G}_{p}\right)\left\|\psi_{z}^{h}\right\|_{H^{2}}^{2} \\
& +C_{\delta} Q(\mathcal{E})\left(\left\|\varepsilon \psi_{t}^{h}\right\|_{L^{2}}^{2}+\left\|\partial_{z} \psi^{h}\right\|_{H^{1}}^{2}\right)+C_{\delta}\left(Q(\mathcal{E})+1+\mathfrak{G}_{p}\right) \mathfrak{H}_{p} \\
& \times\left(\left\|\psi^{h}\right\|_{H^{2}}^{2}+\left\|\varepsilon \psi_{t}^{h}\right\|_{L^{2}}^{2}\right)
\end{aligned}
$$

Therefore, we have shown

$$
\begin{aligned}
I_{5} \lesssim & \delta\left\|\varepsilon \nabla \psi_{t}^{h}\right\|_{L^{2}}^{2}+\delta\left\|\varepsilon \psi_{t}^{h}\right\|_{L^{2}}^{2}+\varepsilon^{2} C_{\delta}\left(Q(\mathcal{E})+\mathfrak{G}_{p}\right)\left\|\psi_{z}^{h}\right\|_{H^{2}}^{2} \\
& +C_{\delta} Q(\mathcal{E})\left(\left\|\varepsilon \psi_{t}^{h}\right\|_{L^{2}}^{2}+\left\|\nabla \psi^{h}\right\|_{H^{1}}^{2}\right) \\
& +C_{\delta}\left(Q(\mathcal{E})+1+\mathfrak{G}_{p}\right) \mathfrak{H}_{p}\left(\left\|\psi^{h}\right\|_{H^{2}}^{2}+\left\|\varepsilon \psi_{t}^{h}\right\|_{L^{2}}^{2}\right) .
\end{aligned}
$$

Next, after substituting (1.7) into $I_{2}$, it follows that

$$
\begin{aligned}
I_{2}= & -\int \varepsilon^{2}\left(\rho \psi^{h} \cdot \nabla_{h} v_{p, t}+\zeta_{t} \psi^{h} \cdot \nabla_{h} v_{p}+\rho \psi_{t}^{h} \cdot \nabla_{h} v_{p}\right) \cdot \psi_{t}^{h} \mathrm{~d} \vec{x} \\
& -\int \varepsilon^{2}\left(\rho \psi^{z} \partial_{z} v_{p, t}+\zeta_{t} \psi^{z} \partial_{z} v_{p}+\rho \psi_{t}^{z} \partial_{z} v_{p}\right) \cdot \psi_{t}^{h} \mathrm{~d} \vec{x}=: I_{2}^{\prime}+I_{2}^{\prime \prime} .
\end{aligned}
$$

Similarly to before,

$$
\begin{aligned}
I_{2}^{\prime} \lesssim & \|\rho\|_{L^{\infty}}\left\|\nabla_{h} v_{p}\right\|_{L^{6}}\left\|\varepsilon \psi_{t}^{h}\right\|_{L^{3}}\left\|\varepsilon \psi_{t}^{h}\right\|_{L^{2}}+\varepsilon\left\|_{\rho}\right\|_{L^{\infty}}\left\|\nabla_{h} v_{p, t}\right\|_{L^{2}} \\
& \times\left\|\psi^{h}\right\|_{L^{6}}\left\|\psi_{t}^{h}\right\|_{L^{3}}+\varepsilon\left\|\nabla_{h} v_{p}\right\|_{L^{\infty}}\left\|\psi^{h}\right\|_{L^{6}}\left\|\zeta_{t}\right\|_{L^{2}}\left\|\varepsilon \psi_{t}^{h}\right\|_{L^{3}} \\
\lesssim & \|\rho\|_{H^{2}}\left\|\nabla_{h} v_{p}\right\|_{L^{2}}\left(\left\|\varepsilon \psi_{t}^{h}\right\|_{L^{2}}^{3 / 2}\left\|\varepsilon \nabla \psi_{t}^{h}\right\|_{L^{2}}^{1 / 2}+\left\|\varepsilon \psi_{t}^{h}\right\|_{L^{2}}^{2}\right) \\
& +\varepsilon\left\|\psi^{h}\right\|_{H^{1}}\left\|\nabla_{h} v_{p, t}\right\|_{L^{2}}\left(\left\|\varepsilon \psi_{t}^{h}\right\|_{L^{2}}^{1 / 2}\left\|\varepsilon \nabla \psi_{t}^{h}\right\|_{L^{2}}^{1 / 2}+\left\|\varepsilon \psi_{t}^{h}\right\|_{L^{2}}\right) \\
& +\varepsilon\left\|\nabla_{h} v_{p}\right\|_{H^{2}}\left\|\psi^{h}\right\|_{H^{1}}\left\|\zeta_{t}\right\|_{L^{2}}\left(\left\|\varepsilon \psi_{t}^{h}\right\|_{L^{2}}^{1 / 2}\left\|\varepsilon \nabla \psi_{t}^{h}\right\|_{L^{2}}^{1 / 2}+\left\|\varepsilon \psi_{t}^{h}\right\|_{L^{2}}\right) \\
& \lesssim \delta\left\|\varepsilon \nabla \psi_{t}^{h}\right\|_{L^{2}}+C_{\delta} Q(\mathcal{E})\left\|\varepsilon \psi_{t}^{h}\right\|_{L^{2}}^{2}+C_{\delta}\left(Q(\mathcal{E})+1+\mathfrak{G}_{p}\right) \mathfrak{H}_{p}\left\|\varepsilon \psi_{t}^{h}\right\|_{L^{2}}^{2} \\
& +\varepsilon^{2} C_{\delta} \mathfrak{H}_{p} .
\end{aligned}
$$

On the other hand, after substituting (3.9), $I_{2}^{\prime \prime}$ can be written as

$$
I_{2}^{\prime \prime}=\int\left[\varepsilon^{2} \int_{0}^{z}\left(\operatorname{div}_{h} \widetilde{\psi^{h}}+\widetilde{v} \cdot \nabla_{h} \log \rho\right) \mathrm{d} z \times\left(\left(\rho \partial_{z} v_{p, t}+\zeta_{t} \partial_{z} v_{p}\right) \cdot \psi_{t}^{h}\right)\right] \mathrm{d} \vec{x}
$$




$$
\begin{aligned}
& +\int\left[\varepsilon^{2} \int_{0}^{z}\left(\operatorname{div}_{h} \widetilde{\psi_{t}^{h}}+\widetilde{v}_{t} \cdot \nabla_{h} \log \rho+\widetilde{v} \cdot \nabla_{h}(\log \rho)_{t}\right) \mathrm{d} z\right. \\
& \left.\times\left(\rho \partial_{z} v_{p} \cdot \psi_{t}^{h}\right)\right] \mathrm{d} \vec{x}=: I_{2,1}^{\prime \prime}+I_{2,2}^{\prime \prime}
\end{aligned}
$$

Then we have the following estimate:

$$
\begin{aligned}
I_{2,1}^{\prime \prime} \lesssim & \int_{0}^{1}\left(\left|\nabla_{h} \psi^{h}\right|_{L^{4}}+|v|_{L^{\infty}}\left|\nabla_{h} \zeta\right|_{L^{4}}\right) \mathrm{d} z \times \int_{0}^{1}\left(\left(\varepsilon\left|\partial_{z} v_{p, t}\right|_{L^{2}}\right.\right. \\
& \left.\left.+\varepsilon\left|\zeta_{t}\right|_{L^{2}}\left|\partial_{z} v_{p}\right|_{L^{\infty}}\right)\left|\varepsilon \psi_{t}^{h}\right|_{L^{4}}\right) \mathrm{d} z \lesssim \delta\left\|\varepsilon \nabla \psi_{t}^{h}\right\|_{L^{2}}^{2} \\
& +C_{\delta} Q(\mathcal{E})\left\|\varepsilon \psi_{t}^{h}\right\|_{L^{2}}^{2}+C_{\delta}\left(Q(\mathcal{E})+1+\mathfrak{G}_{p}\right) \mathfrak{H}_{p}\left\|\varepsilon \psi_{t}^{h}\right\|_{L^{2}}^{2}+\varepsilon^{2} C_{\delta} \mathfrak{H}_{p}
\end{aligned}
$$

To estimate $I_{2,2}^{\prime \prime}$, we first apply integration by parts as follows:

$$
\begin{aligned}
I_{2,2}^{\prime \prime}= & \int\left[\varepsilon^{2} \int_{0}^{z}\left(\rho \operatorname{div}_{h} \widetilde{\psi_{t}^{h}}+\widetilde{v}_{t} \cdot \nabla_{h} \rho-\zeta_{t} \widetilde{v} \cdot \nabla_{h} \log \rho-\zeta_{t} \operatorname{div}_{h} \widetilde{v}\right) \mathrm{d} z\right. \\
& \left.\times\left(\partial_{z} v_{p} \cdot \psi_{t}^{h}\right)\right] \mathrm{d} \vec{x}-\int\left[\varepsilon^{2}\left(\int_{0}^{z} \zeta_{t} \widetilde{v} \mathrm{~d} z\right) \cdot \nabla_{h}\left(\partial_{z} v_{p} \cdot \psi_{t}^{h}\right)\right] \mathrm{d} \vec{x}
\end{aligned}
$$

which yields, similarly to the estimate of $I_{5,3}^{\prime \prime \prime}$,

$$
\begin{aligned}
I_{2,2}^{\prime \prime} \lesssim & \int_{0}^{1}\left(|\rho|_{L^{\infty}}\left|\varepsilon \nabla_{h} \psi_{t}^{h}\right|_{L^{2}}+\left|\varepsilon v_{t}\right|_{L^{4}}\left|\nabla_{h} \zeta\right|_{L^{4}}\right) \mathrm{d} z \\
& \times \int_{0}^{1}\left|\partial_{z} v_{p}\right|_{L^{4}}\left|\varepsilon \psi_{t}^{h}\right|_{L^{4}} \mathrm{~d} z+\int_{0}^{1}\left|\partial_{z} v_{p}\right|_{L^{8}}\left|\varepsilon \psi_{t}^{h}\right|_{L^{4}} \mathrm{~d} z \\
& \times \int_{0}^{1}\left(\varepsilon\left|\zeta_{t}\right|_{L^{2}}|v|_{L^{\infty}}\left|\nabla_{h} \zeta\right|_{L^{8}}+\varepsilon\left|\zeta_{t}\right|_{L^{2}}\left|\nabla_{h} v\right|_{L^{8}}\right) \mathrm{d} z \\
& +\int_{0}^{1}\left(\left|\partial_{z} v_{p}\right|_{L^{\infty}}\left|\varepsilon \nabla_{h} \psi_{t}^{h}\right|_{L^{2}}+\left|\nabla_{h} \partial_{z} v_{p}\right|_{L^{4}}\left|\varepsilon \psi_{t}^{h}\right|_{L^{4}}\right) \mathrm{d} z \\
& \times \int_{0}^{1} \varepsilon\left|\zeta_{t}\right|_{L^{2}}|v|_{L^{\infty}} \mathrm{d} z \\
\lesssim & \delta\left\|\varepsilon \nabla_{h} \psi_{t}^{h}\right\|_{L^{2}}^{2}+C_{\delta} Q(\mathcal{E})\left\|\varepsilon \psi_{t}^{h}\right\|_{L^{2}}^{2}+C_{\delta}\left(Q(\mathcal{E})+1+\mathfrak{G}_{p}\right) \mathfrak{H}_{p} \\
& \times\left(\left\|\varepsilon \psi_{t}^{h}\right\|_{L^{2}}^{2}+\varepsilon^{2}\right) .
\end{aligned}
$$

Therefore,

$$
\begin{aligned}
I_{2} \lesssim & \delta\left\|\varepsilon \nabla \psi_{t}^{h}\right\|_{2}^{2}+C_{\delta} Q(\mathcal{E})\left\|\varepsilon \psi_{t}^{h}\right\|_{2}^{2} \\
& +C_{\delta}\left(Q(\mathcal{E})+1+\mathfrak{G}_{p}\right) \mathfrak{H}_{p}\left(\left\|\varepsilon \psi_{t}^{h}\right\|_{L^{2}}^{2}+\varepsilon^{2}\right)
\end{aligned}
$$


Now, we will estimate $I_{3}$, which reads as

$$
\begin{aligned}
I_{3}= & -\rho_{0}^{-1} c_{s}^{2} \int\left(\xi_{t} \operatorname{div}_{h}\left(\xi_{t} v\right)+\xi_{t} \operatorname{div}_{h}\left(\xi v_{t}\right)+\xi_{t} \partial_{z}\left(\xi_{t} w\right)\right. \\
& \left.+\xi_{t} \partial_{z}\left(\xi w_{t}\right)\right) \mathrm{d} \vec{x}=-\frac{c_{s}^{2}}{2 \rho_{0}} \int\left|\xi_{t}\right|^{2} \operatorname{div}_{h} \psi^{h} \mathrm{~d} \vec{x} \\
& -\rho_{0}^{-1} c_{s}^{2} \int\left(\xi_{t} \xi \operatorname{div}_{h} \psi_{t}^{h}+\xi_{t} v_{t} \cdot \nabla_{h} \xi\right) \mathrm{d} \vec{x} \\
\lesssim & \delta\left\|\varepsilon \nabla \psi_{t}^{h}\right\|_{L^{2}}^{2}+\delta\left\|\varepsilon \psi_{t}^{h}\right\|_{L^{2}}^{2}+\delta\left\|\nabla_{h} \psi^{h}\right\|_{H^{2}}^{2}+C_{\delta} Q(\mathcal{E})\left\|\xi_{t}\right\|_{2}^{2} \\
& +\varepsilon^{2} C_{\delta} \mathfrak{H}_{p} .
\end{aligned}
$$

Here we have employed the facts that $\xi$ is independent of the $z$-variable and that $\int_{0}^{1} \operatorname{div}_{h} v_{p} \mathrm{~d} z=\int_{0}^{1} \operatorname{div}_{h} v_{p, t} \mathrm{~d} z=0$. The rest is straightforward. For instance, substituting (1.7) in $I_{1}$ yields

$$
\begin{aligned}
I_{1}= & \rho_{0}^{-1} \int \varepsilon \zeta_{t}\left(\nabla_{h}\left(c_{s}^{2} \rho_{1}\right)-\mu \Delta_{h} v_{p}-\lambda \nabla_{h} \operatorname{div}_{h} v_{p}-\partial_{z z} v_{p}\right) \cdot \varepsilon \psi_{t}^{h} \mathrm{~d} \vec{x} \\
& +\rho_{0}^{-1} \int \varepsilon \zeta\left(\nabla_{h}\left(c_{s}^{2} \rho_{1, t}\right)-\mu \Delta_{h} v_{p, t}-\lambda \nabla_{h} \operatorname{div}_{h} v_{p, t}-\partial_{z z} v_{p, t}\right) \cdot \varepsilon \psi_{t}^{h} \mathrm{~d} \vec{x} \\
= & \rho_{0}^{-1} \int \varepsilon \zeta_{t}\left(\nabla_{h}\left(c_{s}^{2} \rho_{1}\right)-\mu \Delta_{h} v_{p}-\lambda \nabla_{h} \operatorname{div}_{h} v_{p}-\partial_{z z} v_{p}\right) \cdot \varepsilon \psi_{t}^{h} \mathrm{~d} \vec{x} \\
& -\rho_{0}^{-1} \int\left(\varepsilon c_{s}^{2} \rho_{1, t} \operatorname{div}_{h}\left(\zeta \varepsilon \psi_{t}^{h}\right)-\varepsilon \mu \nabla_{h} v_{p, t}: \nabla_{h}\left(\zeta \varepsilon \psi_{t}^{h}\right)\right. \\
& \left.-\varepsilon \lambda \operatorname{div}_{h} v_{p, t} \operatorname{div}_{h}\left(\zeta \varepsilon \psi_{t}^{h}\right)-\varepsilon \partial_{z} v_{p, t} \cdot \partial_{z}\left(\zeta \varepsilon \psi_{t}^{h}\right)\right) \mathrm{d} \vec{x} \\
\lesssim & \left\|\varepsilon \nabla \psi_{t}^{h}\right\|_{L^{2}}^{2}+\delta\left\|\varepsilon \psi_{t}^{h}\right\|_{L^{2}}^{2}+\varepsilon^{2} C_{\delta}\left(Q(\mathcal{E})+1+\mathfrak{G}_{p}\right) \mathfrak{H}_{p} .
\end{aligned}
$$

We list estimates for $I_{4}, I_{6}$ as follows:

$$
\begin{aligned}
& I_{4}=-\rho_{0}^{-1} c_{s}^{2} \varepsilon^{2} \int\left(\xi_{t}\left(\rho_{1, t t}+\rho_{1, t} \operatorname{div}_{h} \psi^{h}+v \cdot \nabla_{h} \rho_{1, t}\right)\right. \\
&\left.+\xi_{t}\left(\rho_{1} \operatorname{div}_{h} \psi_{t}^{h}+v_{t} \cdot \nabla_{h} \rho_{1}\right)\right) \mathrm{d} \vec{x} \lesssim \delta\left\|\xi_{t}\right\|_{L^{2}}^{2} \\
&+\delta\left\|\varepsilon \nabla_{h} \psi_{t}^{h}\right\|_{L^{2}}^{2}+\delta\left\|\nabla_{h} \psi^{h}\right\|_{H^{2}}+\varepsilon^{2} C_{\delta}\left(Q(\mathcal{E})+1+\mathfrak{G}_{p}\right) \mathfrak{H}_{p}, \\
& I_{6} \lesssim\left\|\varepsilon^{-1} \zeta\right\|_{L^{\infty}}\left\|\zeta_{t}\right\|_{L^{2}}\left\|\varepsilon \nabla_{h} \psi_{t}^{h}\right\|_{L^{2}} \lesssim \delta\left\|\varepsilon \nabla \psi_{t}^{h}\right\|_{L^{2}}^{2} \\
&+C_{\delta}\left(\varepsilon^{2}\left\|\rho_{1}\right\|_{H^{2}}^{2}+\left\|\varepsilon^{-1} \xi\right\|_{H^{2}}^{2}\right)\left(\varepsilon^{4}\left\|\rho_{1, t}\right\|_{2}^{2}+\left\|\xi_{t}\right\|_{2}^{2}\right),
\end{aligned}
$$

where we have used the fact that from (1.6)

$$
\left|\mathcal{R}_{t}\right|=\left|\gamma(\gamma-1) \int_{\rho_{0}}^{\rho} \rho_{t} y^{\gamma-2} d y\right| \leqq\left|\gamma \rho_{t}\left(\rho^{\gamma-1}-\rho_{0}^{\gamma-1}\right)\right| \leqq C\left|\zeta_{t}\right||\zeta| .
$$

Summing up inequalities (3.10), (3.11)-(3.15) and (3.8) completes the proof. 
The next lemma follows directly from system (1.8), and it shows the estimates of the temporal derivatives of $\xi, \psi^{h}$ in terms of the spatial derivatives.

Lemma 2. Under the same assumptions as in Lemma 1,

$$
\begin{aligned}
\left\|\xi_{t}\right\|_{L^{2}}^{2} \leqq & C\left\|\nabla_{h} \psi^{h}\right\|_{L^{2}}^{2}+\varepsilon^{2} Q(\mathcal{E})\left(\left\|\nabla_{h} \psi^{h}\right\|_{H^{1}}^{2}+\left\|\varepsilon^{-1} \nabla_{h} \xi\right\|_{H^{1}}^{2}\right) \\
& +\varepsilon^{2} C\left(Q(\mathcal{E})+1+\mathfrak{G}_{p}\right) \mathfrak{H}_{p}, \\
\left\|\varepsilon \rho \psi_{t}^{h}\right\|_{L^{2}}^{2} \leqq & C\left\|\varepsilon^{-1} \nabla_{h} \xi\right\|_{L^{2}}^{2}+\varepsilon^{2} C\left\|\nabla \psi^{h}\right\|_{H^{1}}^{2}+\varepsilon^{2} Q(\mathcal{E})\left(\left\|\varepsilon^{-1} \nabla_{h} \xi\right\|_{H^{1}}^{2}\right. \\
& \left.+\left\|\nabla \psi^{h}\right\|_{H^{1}}^{2}\right)+\varepsilon^{2} C\left(Q(\mathcal{E})+1+\mathfrak{G}_{p}\right) \mathfrak{H}_{p},
\end{aligned}
$$

for some positive constant $C$ independent of $\varepsilon$.

Proof. Indeed, after integrating $(1.8)_{1}$ in the $z$ variable, we have, thanks to (1.5),

$$
\partial_{t} \xi+\rho_{0} \operatorname{div}_{h} \overline{\psi^{h}}=-\operatorname{div}_{h}(\xi \bar{v})-\varepsilon^{2}\left(\partial_{t} \rho_{1}+\operatorname{div}_{h}\left(\rho_{1} \bar{v}\right)\right) .
$$

Then (3.16) follows easily after applying the Minkowski, Hölder and Sobolev embedding inequalities.

On the other hand, (3.17) follows from (1.8) 2 after substituting

$$
\rho w \partial_{z} \psi^{h}=\rho w_{p} \partial_{z} \psi^{h}-\int_{0}^{z} \rho \operatorname{div}_{h} \widetilde{\psi}^{h}+\widetilde{v} \cdot \nabla_{h} \rho \mathrm{d} z \partial_{z} \psi^{h},
$$

and applying the Minkowski, Hölder and Sobolev embedding inequalities.

\subsection{Horizontal Derivatives}

We derive the required estimates for the horizontal derivatives in this subsection. After applying $\partial_{h h}=\partial_{h}^{2}$ to system (1.8), we obtain the following system:

$$
\left\{\begin{array}{cc}
\partial_{t} \xi_{h h}+\rho_{0}\left(\operatorname{div}_{h} \psi_{h h}^{h}+\partial_{z} \psi_{h h}^{z}\right)=\mathcal{G}_{1, h h}+\mathcal{G}_{2, h h} & \text { in } \Omega, \\
\rho \partial_{t} \psi_{h h}^{h}+\rho v \cdot \nabla_{h} \psi_{h h}^{h}+\rho w \partial_{z} \psi_{h h}^{h}+\nabla_{h}\left(\varepsilon^{-2} c_{s}^{2} \xi_{h h}\right) & \\
=\mu \Delta_{h} \psi_{h h}^{h}+\lambda \nabla_{h} \operatorname{div}_{h} \psi_{h h}^{h}+\partial_{z z} \psi_{h h}^{h}+\mathcal{F}_{1, h h} & \\
\quad+\mathcal{F}_{2, h h}-\nabla_{h}\left(\varepsilon^{-2} \mathcal{R}_{h h}\right)+\mathcal{H}_{h h} & \text { in } \Omega,
\end{array}\right.
$$

where

$$
\begin{aligned}
\mathcal{H}_{h h}:= & -\rho_{h h} \psi_{t}^{h}-(\rho v)_{h h} \cdot \nabla_{h} \psi^{h}-(\rho w)_{h h} \partial_{z} \psi^{h}-2 \rho_{h} \psi_{h t}^{h} \\
& -2(\rho v)_{h} \cdot \nabla_{h} \psi_{h}^{h}-2(\rho w)_{h} \partial_{z} \psi_{h}^{h} .
\end{aligned}
$$

Lemma 3. Under the same assumptions as in Lemma 1, we have

$$
\begin{aligned}
\frac{d}{\mathrm{~d} t}\{ & \left.\frac{1}{2}\left\|\rho^{1 / 2} \psi_{h h}^{h}\right\|_{L^{2}}^{2}+\frac{c_{s}^{2}}{2 \rho_{0}}\left\|\varepsilon^{-1} \xi_{h h}\right\|_{L^{2}}^{2}\right\}+C_{\mu, \lambda}\left\|\nabla \psi_{h h}^{h}\right\|_{L^{2}}^{2} \\
\leqq & \delta\left(\left\|\nabla \psi^{h}\right\|_{H^{2}}^{2}+\left\|\varepsilon^{-1} \nabla_{h} \xi\right\|_{H^{1}}^{2}+\left\|\varepsilon \nabla \psi_{t}^{h}\right\|_{L^{2}}^{2}\right. \\
& \left.+\left\|\varepsilon \psi_{t}^{h}\right\|_{L^{2}}^{2}\right)+C_{\delta} Q(\mathcal{E})\left(\left\|\nabla \psi^{h}\right\|_{H^{1}}^{2}+\left\|\varepsilon^{-1} \nabla_{h} \xi\right\|_{H^{1}}^{2}\right) \\
& +C_{\delta}\left(Q(\mathcal{E})+1+\mathfrak{G}_{p}\right) \mathfrak{H}_{p}\left(\left\|\psi^{h}\right\|_{H^{2}}^{2}+\left\|\varepsilon^{-1} \xi\right\|_{H^{2}}^{2}+\varepsilon^{2}\right)
\end{aligned}
$$

for some positive constant $C_{\mu, \lambda}$, which is independent of $\varepsilon$. 
Proof. Take the inner product of $(3.18)_{2}$ with $\psi_{h h}^{h}$ and integrate the resultant over $\Omega$. Similarly to before, we will have the following:

$$
\begin{aligned}
& \frac{d}{\mathrm{~d} t}\left\{\frac{1}{2}\left\|\rho^{1 / 2} \psi_{h h}^{h}\right\|_{L^{2}}^{2}+\frac{c_{s}^{2}}{2 \rho_{0}}\left\|\varepsilon^{-1} \xi_{h h}\right\|_{L^{2}}^{2}\right\}+\mu\left\|\nabla_{h} \psi_{h h}^{h}\right\|_{L^{2}}^{2} \\
& \quad+\lambda\left\|\operatorname{div}_{h} \psi_{h h}^{h}\right\|_{L^{2}}^{2}+\left\|\partial_{z} \psi_{h h}^{h}\right\|_{L^{2}}^{2}=\int \mathcal{F}_{1, h h} \cdot \psi_{h h}^{h} \mathrm{~d} \vec{x} \\
& \quad+\int \mathcal{F}_{2, h h} \cdot \psi_{h h}^{h} \mathrm{~d} \vec{x}+\rho_{0}^{-1} c_{s}^{2} \int \varepsilon^{-2} \xi_{h h} \mathcal{G}_{1, h h} \mathrm{~d} \vec{x} \\
& \quad+\rho_{0}^{-1} c_{s}^{2} \int \varepsilon^{-2} \xi_{h h} \mathcal{G}_{2, h h} \mathrm{~d} \vec{x}+\int \mathcal{H}_{h h} \cdot \psi_{h h}^{h} \mathrm{~d} \vec{x} \\
& \quad-\int \nabla_{h}\left(\varepsilon^{-2} \mathcal{R}_{h h}\right) \cdot \psi_{h h}^{h} \mathrm{~d} \vec{x}=: \sum_{i=7}^{12} I_{i} .
\end{aligned}
$$

Then the lemma follows from careful estimates on the right-hand side of (3.21), which are similar to those in the proof of Lemma 1 . Therefore, details are omitted here.

Next, we will derive the required estimate of $\xi_{h h}$. After integrating (1.8) 2 over $z \in(0,1)$, we have the following equation, thanks to $(1.5)$ :

$$
\begin{aligned}
& \rho \partial_{t} \overline{\psi^{h}}+\int_{0}^{1}\left(\rho v \cdot \nabla_{h} \psi^{h}-\rho w_{z} \psi^{h}\right) \mathrm{d} z+\nabla_{h}\left(\varepsilon^{-2} c_{s}^{2} \xi\right) \\
& =\mu \Delta_{h} \overline{\psi^{h}}+\lambda \nabla_{h} \operatorname{div}_{h} \overline{\psi^{h}}-\nabla_{h}\left(\varepsilon^{-2} \mathcal{R}\right)+\int_{0}^{1}\left(\mathcal{F}_{1}+\mathcal{F}_{2}\right) \mathrm{d} z
\end{aligned}
$$

After applying $\partial_{h}$ to (3.22), one has

$$
\begin{aligned}
\varepsilon^{-2} c_{s}^{2} \nabla_{h} \xi_{h}= & \underbrace{-\zeta_{h} \partial_{t} \overline{\psi^{h}}-\rho \partial_{t} \overline{\psi_{h}^{h}}}_{R_{1}} \\
& \underbrace{-\int_{0}^{1}\left(\zeta_{h} v \cdot \nabla_{h} \psi^{h}+\rho v_{h} \cdot \nabla_{h} \psi^{h}+\rho v \cdot \nabla_{h} \psi_{h}^{h}\right) \mathrm{d} z}_{R_{2}} \\
& +\underbrace{\int_{0}^{1}\left(\zeta_{h} w_{z} \psi^{h}+\rho w_{h z} \psi^{h}+\rho w_{z} \psi_{h}^{h}\right) \mathrm{d} z}_{R_{3}} \\
& +\underbrace{\mu \Delta_{h} \overline{\psi_{h}^{h}}+\lambda \nabla_{h} \operatorname{div}_{h} \overline{\psi_{h}^{h}}-\nabla_{h}\left(\varepsilon^{-2} \mathcal{R}_{h}\right)}_{R_{4}}+\underbrace{\int_{0}^{1}\left(\mathcal{F}_{1, h}+\mathcal{F}_{2, h}\right) \mathrm{d} z}_{R_{5}} .
\end{aligned}
$$

What we need is to estimate the $L^{2}$-norm of the terms on the right-hand side of (3.23). In fact, after applying the Minkowski, Hölder and Sobolev embedding 
inequalities, one has

$$
\begin{aligned}
\left|R_{1}\right|_{L^{2}} \lesssim & \int_{0}^{1}\left|\zeta_{h}\right|_{L^{4}}\left|\partial_{t} \psi^{h}\right|_{L^{4}} \mathrm{~d} z+\int_{0}^{1}|\rho|_{L^{\infty}}\left|\partial_{t} \psi_{h}^{h}\right|_{L^{2}} \mathrm{~d} z \lesssim\left\|\zeta_{h}\right\|_{H^{1}} \\
& \times\left(\left\|\partial_{t} \psi^{h}\right\|_{L^{2}}^{1 / 2}\left\|\nabla \partial_{t} \psi^{h}\right\|_{L^{2}}^{1 / 2}+\left\|\partial_{t} \psi^{h}\right\|_{L^{2}}\right)+\|\rho\|_{L^{\infty}}\left\|\partial_{t} \psi_{h}^{h}\right\|_{L^{2}} \\
\lesssim & \left\|\nabla \partial_{t} \psi^{h}\right\|_{L^{2}}+\left(\left\|\varepsilon^{-1} \xi\right\|_{H^{2}}+\mathfrak{G}_{p}\left\|\varepsilon \rho_{1}\right\|_{H^{2}}\right)\left\|\varepsilon \partial_{t} \psi^{h}\right\|_{L^{2}}, \\
\left|R_{2}\right|_{L^{2}} \lesssim & \int_{0}^{1}\left(\left|\zeta_{h}\right|_{L^{4}}|v|_{L^{\infty}}\left|\nabla_{h} \psi^{h}\right|_{L^{4}}+|\rho|_{L^{\infty}}\left|v_{h}\right|_{L^{4}}\left|\nabla_{h} \psi^{h}\right|_{L^{4}}+|\rho|_{L^{\infty}}\right. \\
& \left.\times|v|_{L^{\infty}}\left|\nabla_{h} \psi_{h}^{h}\right|_{L^{2}}\right) \mathrm{d} z \lesssim\left(\|\zeta\|_{H^{2}}+\|\rho\|_{L^{\infty}}\right)\|v\|_{H^{2}}\left\|\nabla_{h} \psi^{h}\right\|_{H^{1}}, \\
\left|R_{4}\right|_{L^{2}} \lesssim & \int_{0}^{1}\left|\nabla_{h} \psi^{h}\right|_{H^{2}} \mathrm{~d} z+\varepsilon^{-2}\left(\left|\zeta_{h h}\right|_{L^{2}}|\zeta|_{L^{\infty}}+\left|\zeta_{h}\right|_{L^{4}}^{2}\right) \lesssim\left\|\nabla_{h} \psi^{h}\right\|_{H^{2}} \\
& +\varepsilon^{2}\left\|\rho_{1}\right\|_{H^{2}}^{2}+\left\|\varepsilon^{-1} \xi\right\|_{H^{2}}\left\|\varepsilon^{-1} \nabla_{h} \xi\right\|_{H^{1}} .
\end{aligned}
$$

On the other hand, after substituting (3.33), and the identity

$$
w_{h z}=w_{p, h z}-\left(\operatorname{div}_{h} \widetilde{\psi_{h}^{h}}+\widetilde{v}_{h} \cdot \nabla_{h} \log \rho+\widetilde{v} \cdot \nabla_{h}(\log \rho)_{h}\right)=w_{p, h z}+\psi_{h z}^{z},
$$

we have

$$
\begin{aligned}
R_{3}= & \int_{0}^{1}\left(\left[w_{p, z}-\left(\operatorname{div}_{h} \widetilde{\psi^{h}}+\widetilde{v} \cdot \nabla_{h} \log \rho\right)\right]\left(\zeta_{h} \psi^{h}+\rho \psi_{h}^{h}\right)+\rho \psi^{h}\left[w_{p, h z}\right.\right. \\
& \left.\left.-\left(\operatorname{div}_{h} \widetilde{\psi_{h}^{h}}+\widetilde{v}_{h} \cdot \nabla_{h} \log \rho+\widetilde{v} \cdot \nabla_{h}(\log \rho)_{h}\right)\right]\right) \mathrm{d} z .
\end{aligned}
$$

Therefore, one has

$$
\begin{aligned}
\left|R_{3}\right|_{L^{2}} \lesssim \int_{0}^{1}\left(\left(\left|w_{p, z}\right|_{L^{4}}+\left|\nabla_{h} \psi^{h}\right|_{L^{4}}+|v|_{L^{\infty}}\left|\nabla_{h} \zeta\right|_{L^{4}}\right)\right. \\
\quad \times\left(\left|\zeta_{h}\right|_{L^{4}}\left|\psi^{h}\right|_{L^{\infty}}+|\rho|_{L^{\infty}}\left|\psi_{h}^{h}\right|_{L^{4}}\right)+|\rho|_{L^{\infty}}\left|\psi^{h}\right|_{L^{\infty}} \\
\quad \times\left(\left|w_{p, h z}\right|_{L^{2}}+\left|\nabla_{h} \psi_{h}^{h}\right|_{L^{2}}+\left|v_{h}\right|_{L^{4}}\left|\nabla_{h} \zeta\right|_{L^{4}}\right. \\
\left.\left.\quad+|v|_{L^{\infty}}\left(\left|\zeta_{h h}\right|_{L^{2}}+\left|\zeta_{h}\right|_{L^{4}}^{2}\right)\right)\right) \mathrm{d} z \\
\lesssim Q(\mathcal{E})\left(\left\|\nabla_{h} \psi^{h}\right\|_{H^{1}}+\varepsilon\left\|\varepsilon^{-1} \nabla_{h} \xi\right\|_{H^{1}}\right)+\left(Q(\mathcal{E})+1+\mathfrak{G}_{p}\right) \mathfrak{H}_{p}^{1 / 2} .
\end{aligned}
$$

After substituting (1.7) and (1.10) and applying integration by parts, we obtain

$$
\begin{aligned}
R_{5}= & \int_{0}^{1}\left(\zeta_{h} Q_{p}+\zeta Q_{p, h}-\zeta_{h} \psi^{h} \cdot \nabla_{h} v_{p}-\rho \psi_{h}^{h} \cdot \nabla_{h} v_{p}-\rho \psi^{h} \cdot \nabla_{h} v_{p, h}\right. \\
& -\left(\operatorname{div}_{h} \widetilde{\psi^{h}}+\widetilde{v} \cdot \nabla_{h} \log \rho\right)\left(\zeta_{h} v_{p}+\rho v_{p, h}\right) \\
& \left.-\left(\operatorname{div}_{h} \widetilde{\psi_{h}^{h}}+\widetilde{v}_{h} \cdot \nabla_{h} \log \rho+\widetilde{v} \cdot \nabla_{h}(\log \rho)_{h}\right) \rho v_{p}\right) \mathrm{d} z
\end{aligned}
$$


Hence, applying the Minkowski, Hölder and Sobolev embedding inequalities implies

$$
\left|R_{5}\right|_{L^{2}} \lesssim\left(Q(\mathcal{E})+1+\mathfrak{G}_{p}\right) \mathfrak{H}_{p}^{1 / 2}\left(\left\|\psi^{h}\right\|_{H^{2}}+\varepsilon\right) .
$$

Summing up these estimates, we get the following inequality from (3.23),

$$
\begin{aligned}
\left\|\varepsilon^{-1} \xi_{h h}\right\|_{L^{2}}^{2} \lesssim & \varepsilon^{2}\left|\varepsilon^{-2} \xi_{h h}\right|_{L^{2}}^{2} \lesssim\left\|\varepsilon \nabla \partial_{t} \psi^{h}\right\|_{L^{2}}^{2} \\
& +\varepsilon^{2}\left\|\nabla \psi^{h}\right\|_{H^{2}}^{2}+\varepsilon^{2} Q(\mathcal{E})\left(\left\|\varepsilon^{-1} \nabla_{h} \xi\right\|_{H^{1}}^{2}+\left\|\nabla \psi^{h}\right\|_{H^{1}}^{2}\right. \\
& \left.+\left\|\varepsilon \partial_{t} \psi^{h}\right\|_{L^{2}}^{2}\right)+\varepsilon^{2}\left(Q(\mathcal{E})+1+\mathfrak{G}_{p}\right) \mathfrak{H}_{p} .
\end{aligned}
$$

We summarize the result in the following:

Lemma 4. Under the same assumptions as in Lemma 1, the following holds:

$$
\begin{aligned}
& \left\|\varepsilon^{-1} \nabla_{h} \xi\right\|_{H^{1}}^{2} \\
& \leq C\left\|\varepsilon \nabla \partial_{t} \psi^{h}\right\|_{L^{2}}^{2}+\varepsilon^{2}\left\|\nabla \psi^{h}\right\|_{H^{2}}^{2} \\
& \quad+\varepsilon^{2} C Q(\mathcal{E})\left(\left\|\varepsilon^{-1} \nabla_{h} \xi\right\|_{H^{1}}^{2}+\left\|\nabla \psi^{h}\right\|_{H^{1}}^{2}+\left\|\varepsilon \psi_{t}^{h}\right\|_{L^{2}}^{2}\right) \\
& \quad+C \varepsilon^{2}\left(Q(\mathcal{E})+1+\mathfrak{G}_{p}\right) \mathfrak{H}_{p},
\end{aligned}
$$

for some positive constant $C$ independent of $\varepsilon$.

Proof. This is the direct consequence of (3.25) and the Poincaré inequality.

\subsection{Vertical Derivatives Estimates}

Now we turn to the required estimates of vertical derivatives. To do so, we first apply $\partial_{z}$ to system (1.8) and write down the resultant system as follows:

$$
\left\{\begin{array}{lc}
\rho_{0}\left(\operatorname{div}_{h} \psi_{z}^{h}+\partial_{z} \psi_{z}^{h}\right)=\mathcal{G}_{1, z}+\mathcal{G}_{2, z} & \text { in } \Omega, \\
\rho \partial_{t} \psi_{z}^{h}+\rho v \cdot \nabla_{h} \psi_{z}^{h}+\rho w \partial_{z} \psi_{z}^{h}=\mu \Delta_{h} \psi_{z}^{h}+\lambda \nabla_{h} \operatorname{div}_{h} \psi_{z}^{h} & \\
\quad+\partial_{z z} \psi_{z}^{h}+\mathcal{F}_{1, z}+\mathcal{F}_{2, z}+\mathcal{H}_{z} & \text { in } \Omega,
\end{array}\right.
$$

where

$$
\mathcal{H}_{z}:=-\rho v_{z} \cdot \nabla_{h} \psi^{h}-\rho w_{z} \partial_{z} \psi^{h} .
$$

Then we apply $\partial_{z}$ to system (3.27) again and obtain the following system:

$$
\begin{cases}\rho_{0}\left(\operatorname{div}_{h} \psi_{z z}^{h}+\partial_{z} \psi_{z z}^{h}\right)=\mathcal{G}_{1, z z}+\mathcal{G}_{2, z z} & \text { in } \Omega, \\ \rho \partial_{t} \psi_{z z}^{h}+\rho v \cdot \nabla_{h} \psi_{z z}^{h}+\rho w \partial_{z} \psi_{z z}^{h}=\mu \Delta_{h} \psi_{z z}^{h}+\lambda \nabla_{h} \operatorname{div}_{h} \psi_{z z}^{h} & \\ \quad+\partial_{z z} \psi_{z z}^{h}+\mathcal{F}_{1, z z}+\mathcal{F}_{2, z z}+\mathcal{H}_{z z} & \text { in } \Omega,\end{cases}
$$

where

$$
\mathcal{H}_{z z}:=-\rho v_{z z} \cdot \nabla_{h} \psi^{h}-2 \rho v_{z} \cdot \nabla_{h} \psi_{z}^{h}-\rho w_{z z} \partial_{z} \psi^{h}-2 \rho w_{z} \partial_{z} \psi_{z}^{h} .
$$

Notice, here we have employed the fact that $\rho, \xi, \rho_{1}, \mathcal{R}$ are independent of the $z$ variable. Also (3.29) $)_{2}$ is a parabolic equation of $\psi_{z z}^{h}$. Now we perform standard $L^{2}$ estimate on system (3.29). 
Lemma 5. Under the same assumptions as in Lemma 1, we have

$$
\begin{aligned}
& \frac{d}{\mathrm{~d} t}\left\|\rho^{1 / 2} \psi_{z z}^{h}\right\|_{L^{2}}^{2}+C_{\mu, \lambda}\left\|\nabla \psi_{z z}^{h}\right\|_{L^{2}}^{2} \\
& \quad \leqq \delta\left\|\nabla \psi_{z z}^{h}\right\|_{L^{2}}^{2}+\delta\left\|\psi_{z z}^{h}\right\|_{L^{2}}^{2} \\
& \quad+C_{\delta} Q(\mathcal{E})\left\|\nabla \psi^{h}\right\|_{H^{1}}^{2}+C_{\delta}\left(Q(\mathcal{E})+1+\mathfrak{G}_{p}\right) \mathfrak{H}_{p}\left(\left\|\psi^{h}\right\|_{H^{2}}^{2}+\varepsilon^{2}\right)
\end{aligned}
$$

for some positive constant $C_{\mu, \lambda}$ independent of $\varepsilon$.

Proof. After taking the $L^{2}$-inner product of $(3.29)_{2}$ with $\psi_{z z}^{h}$, we have the following:

$$
\begin{aligned}
& \frac{d}{\mathrm{~d} t}\left\{\frac{1}{2}\left\|\rho^{1 / 2} \psi_{z z}^{h}\right\|_{L^{2}}^{2}\right\}+\mu\left\|\nabla_{h} \psi_{z z}^{h}\right\|_{L^{2}}^{2}+\lambda\left\|\operatorname{div}_{h} \psi_{z z}^{h}\right\|_{L^{2}}^{2} \\
& \quad+\left\|\partial_{z} \psi_{z z}^{h}\right\|_{L^{2}}^{2}=\int \mathcal{F}_{1, z z} \cdot \psi_{z z}^{h} \mathrm{~d} \vec{x}+\int \mathcal{F}_{2, z z} \cdot \psi_{z z}^{h} \mathrm{~d} \vec{x} \\
& \quad+\int \mathcal{H}_{z z} \cdot \psi_{z z}^{h} \mathrm{~d} \vec{x}=: I_{13}+I_{14}+I_{15} .
\end{aligned}
$$

Again, we shall estimate the terms on the right-hand side of (3.32). We begin with the term $I_{15}$. Notice first, after taking $\partial_{z}, \partial_{z z}$ to (1.9), we have the following identities:

$$
\begin{aligned}
w_{z} & =w_{p, z}-\left(\operatorname{div}_{h} \widetilde{\psi^{h}}+\widetilde{v} \cdot \nabla_{h} \log \rho\right)=w_{p, z}+\psi_{z}^{z}, \\
w_{z z} & =w_{p, z z}-\left(\operatorname{div}_{h} \widetilde{\psi_{z}^{h}}+\widetilde{v}_{z} \cdot \nabla_{h} \log \rho\right)=w_{p, z z}+\psi_{z z}^{z} .
\end{aligned}
$$

Consequently, after substituting (3.33) in $I_{15}$, we have that:

$$
\begin{aligned}
I_{15} \lesssim & \|\rho\|_{L^{\infty}}\left\|\psi_{z z}^{h}\right\|_{L^{3}}\left(\left\|v_{z z}\right\|_{L^{2}}\left\|\nabla_{h} \psi^{h}\right\|_{L^{6}}+\left\|v_{z}\right\|_{L^{6}}\left\|\nabla_{h} \psi_{z}^{h}\right\|_{L^{2}}\right. \\
& +\left\|w_{p, z z}\right\|_{L^{2}}\left\|\partial_{z} \psi^{h}\right\|_{L^{6}}+\left\|\nabla_{h} \psi_{z}^{h}\right\|_{L^{2}}\left\|\partial_{z} \psi^{h}\right\|_{L^{6}} \\
& +\left\|\nabla_{h} \zeta\right\|_{L^{3}}\left\|v_{z}\right\|_{L^{6}}\left\|\partial_{z} \psi^{h}\right\|_{L^{6}}+\left\|w_{p, z}\right\|_{L^{6}}\left\|\partial_{z} \psi_{z}^{h}\right\|_{L^{2}} \\
& \left.+\left\|\nabla_{h} \psi^{h}\right\|_{L^{6}}\left\|\partial_{z} \psi_{z}^{h}\right\|_{L^{2}}+\left\|\nabla_{h} \zeta\right\|_{L^{6}}\|v\|_{L^{\infty}}\left\|\partial_{z} \psi_{z}^{h}\right\|_{L^{2}}\right) \\
\lesssim & \delta \nabla \psi_{z z}^{h}\left\|_{L^{2}}^{2}+\delta\right\| \psi_{z z}^{h} \|_{L^{2}}^{2} \\
& +C_{\delta} Q(\mathcal{E})\left\|\nabla \psi^{h}\right\|_{H^{1}}^{2}+C_{\delta}\left(Q(\mathcal{E})+1+\mathfrak{G}_{p}\right) \mathfrak{H}_{p}\left\|\psi^{h}\right\|_{H^{2}}^{2} .
\end{aligned}
$$

The estimates of the terms $I_{13}, I_{14}$ are as follows:

$$
\begin{aligned}
I_{13}= & \int \zeta Q_{p, z z} \cdot \psi_{z z}^{h} \mathrm{~d} \vec{x}=-\int \zeta Q_{p, z} \cdot \psi_{z z z}^{h} \mathrm{~d} \vec{x} \lesssim \delta\left\|\psi_{z z z}^{h}\right\|_{L^{2}}^{2} \\
& +C_{\delta}\left(Q(\mathcal{E})+\mathfrak{G}_{p}\right) \mathfrak{H}_{p} \varepsilon^{2} \\
I_{14} \lesssim & \delta\left\|\nabla \psi_{z z}^{h}\right\|_{L^{2}}^{2}+\delta\left\|\psi_{z z}^{h}\right\|_{L^{2}}^{2} \\
& +C_{\delta}\left(Q(\mathcal{E})+1+\mathfrak{G}_{p}\right) \mathfrak{H}_{p}\left(\left\|\psi^{h}\right\|_{H^{2}}^{2}+\varepsilon^{2}\left\|\varepsilon^{-1} \xi\right\|_{H^{2}}^{2}+\varepsilon^{4}\right) .
\end{aligned}
$$

After summing the estimates for $I_{13}, I_{14}, I_{15}$, above, and (3.32), we conclude (3.31). 


\subsection{Mixed Horizontal and Vertical Derivatives Estimates}

What is left is to estimate the $L^{2}$ norm of $\partial_{h z} \psi^{h}$. We apply $\partial_{h}$ to (3.27) and write down the resultant system:

$$
\begin{cases}\rho_{0}\left(\operatorname{div}_{h} \psi_{h z}^{h}+\partial_{z} \psi_{h z}^{h}\right)=\mathcal{G}_{1, h z}+\mathcal{G}_{2, h z} & \text { in } \Omega \\ \rho \partial_{t} \psi_{h z}^{h}+\rho v \cdot \nabla_{h} \psi_{h z}^{h}+\rho w \partial_{z} \psi_{h z}^{h}=\mu \Delta_{h} \psi_{h z}^{h} & \\ \quad+\lambda \nabla_{h} \operatorname{div}_{h} \psi_{h z}^{h}+\partial_{z z} \psi_{h z}^{h}+\mathcal{F}_{1, h z}+\mathcal{F}_{2, h z}+\mathcal{H}_{h z} & \text { in } \Omega\end{cases}
$$

where

$$
\begin{aligned}
\mathcal{H}_{h z}: & =-\zeta_{h} \partial_{t} \psi_{z}^{h}-\zeta_{h} v_{z} \cdot \nabla_{h} \psi^{h}-\rho\left(v_{z} \cdot \nabla_{h} \psi^{h}\right)_{h}-\zeta_{h} w_{z} \partial_{z} \psi^{h} \\
& -\rho\left(w_{z} \partial_{z} \psi^{h}\right)_{h}-\zeta_{h} v \cdot \nabla_{h} \psi_{z}^{h}-\rho v_{h} \cdot \nabla_{h} \psi_{z}^{h}-\zeta_{h} w \partial_{z} \psi_{z}^{h} \\
& -\rho w_{h} \partial_{z} \psi_{z}^{h} .
\end{aligned}
$$

Lemma 6. Under the same assumptions as in Lemma 1, we have

$$
\begin{aligned}
\frac{d}{\mathrm{~d} t} & \left\|\rho^{1 / 2} \psi_{h z}^{h}\right\|_{L^{2}}^{2}+C_{\mu, \lambda}\left\|\nabla \psi_{h z}^{h}\right\|_{L^{2}}^{2} \\
\leqq & \delta\left(\left\|\nabla^{3} \psi^{h}\right\|_{L^{2}}^{2}+\left\|\psi_{h z}^{h}\right\|_{L^{2}}^{2}\right. \\
& \left.+\left\|\varepsilon \nabla \partial_{t} \psi^{h}\right\|_{L^{2}}^{2}\right)+C_{\delta} Q(\mathcal{E})\left\|\nabla \psi^{h}\right\|_{H^{1}}^{2} \\
& +C_{\delta}\left(Q(\mathcal{E})+1+\mathfrak{G}_{p}\right) \mathfrak{H}_{p}\left(\varepsilon^{2}+\left\|\psi^{h}\right\|_{H^{2}}^{2}\right),
\end{aligned}
$$

for some positive constant $C_{\mu, \lambda}$, which is independent of $\varepsilon$.

Proof. Take the $L^{2}$ inner produce of $(3.34)_{2}$ with $\psi_{h z}^{h}$. It follows that

$$
\begin{aligned}
& \frac{d}{\mathrm{~d} t}\left\{\frac{1}{2}\left\|\rho^{1 / 2} \psi_{h z}^{h}\right\|_{L^{2}}^{2}\right\}+\mu\left\|\nabla_{h} \psi_{h z}^{h}\right\|_{L^{2}}^{2}+\lambda\left\|\operatorname{div}_{h} \psi_{h z}^{h}\right\|_{L^{2}}^{2} \\
& \quad+\left\|\partial_{z} \psi_{h z}^{h}\right\|_{L^{2}}^{2}=\int \mathcal{F}_{1, h z} \cdot \psi_{h z}^{h} \mathrm{~d} \vec{x}+\int \mathcal{F}_{2, h z} \cdot \psi_{h z}^{h} \mathrm{~d} \vec{x} \\
& \quad+\int \mathcal{H}_{h z} \cdot \psi_{h z}^{h} \mathrm{~d} \vec{x}=: I_{16}+I_{17}+I_{18} .
\end{aligned}
$$

Then the lemma follows from careful estimates of the right-hand side of (3.37), which we omit here again, since they are similar to those we have done before.

\subsection{Proof of Proposition 1}

After applying similar arguments as in the proofs of Lemmas 3, 5 and 6, one can easily check that the following inequalities hold:

$$
\begin{aligned}
& \frac{d}{\mathrm{~d} t}\left\{\frac{1}{2}\left\|\rho^{1 / 2} \psi^{h}\right\|_{L^{2}}^{2}+\frac{c_{s}^{2}}{2 \rho_{0}}\left\|\varepsilon^{-1} \xi\right\|_{L^{2}}^{2}\right\}+C_{\mu, \delta}\left\|\nabla \psi^{h}\right\|_{L^{2}}^{2} \\
& \quad \leqq \delta\left(\left\|\nabla \psi^{h}\right\|_{L^{2}}^{2}+\left\|\varepsilon^{-1} \nabla_{h} \xi\right\|_{H^{1}}^{2}\right)+C_{\delta} Q(\mathcal{E})\left(\left\|\nabla \psi^{h}\right\|_{H^{1}}^{2}\right.
\end{aligned}
$$




$$
\begin{aligned}
&\left.+\left\|\varepsilon^{-1} \nabla_{h} \xi\right\|_{H^{1}}^{2}\right)+C_{\delta}\left(Q(\mathcal{E})+1+\mathfrak{G}_{p}\right) \mathfrak{H}_{p}\left(\varepsilon^{2}+\left\|\psi^{h}\right\|_{H^{2}}^{2}\right) ; \\
& \frac{d}{\mathrm{~d} t}\left\{\frac{1}{2}\left\|\rho^{1 / 2} \psi_{h}^{h}\right\|_{L^{2}}^{2}+\frac{c_{s}^{2}}{2 \rho_{0}}\left\|\varepsilon^{-1} \xi_{h}\right\|_{L^{2}}^{2}\right\}+C_{\mu, \lambda}\left\|\nabla \psi_{h}^{h}\right\|_{L^{2}}^{2} \\
& \leqq \delta\left(\left\|\nabla^{2} \psi^{h}\right\|_{L^{2}}^{2}+\left\|\varepsilon^{-1} \nabla_{h} \xi\right\|_{H^{1}}^{2}+\left\|\varepsilon \psi_{t}^{h}\right\|_{L^{2}}^{2}\right) \\
&+C_{\delta} Q(\mathcal{E})\left(\left\|\nabla \psi^{h}\right\|_{H^{1}}^{2}+\left\|\varepsilon^{-1} \nabla_{h} \xi\right\|_{H^{1}}^{2}\right) \\
&+C_{\delta}\left(Q(\mathcal{E})+1+\mathfrak{G}_{p}\right) \mathfrak{H}_{p}\left(\varepsilon^{2}+\left\|\psi^{h}\right\|_{H^{2}}^{2}\right) ; \\
& \frac{d}{\mathrm{~d} t}\left\|\rho^{1 / 2} \psi_{z}^{h}\right\|_{L^{2}}^{2}+C_{\mu, \lambda}\left\|\nabla \psi_{z}^{h}\right\|_{L^{2}}^{2} \leqq \delta\left(\left\|\nabla^{2} \psi^{h}\right\|_{L^{2}}^{2}+\left\|\psi_{z}^{h}\right\|_{L^{2}}^{2}\right. \\
&\left.\quad+\left\|\varepsilon \partial_{t} \psi^{h}\right\|_{L^{2}}^{2}\right)+C_{\delta} Q(\mathcal{E})\left\|\nabla \psi^{h}\right\|_{H^{1}}^{2} \\
& \quad+C_{\delta}\left(Q(\mathcal{E})+1+\mathfrak{G}_{p}\right) \mathfrak{H}_{p}\left(\varepsilon^{2}+\left\|\psi^{h}\right\|_{H^{2}}^{2}\right) .
\end{aligned}
$$

Therefore, the above inequalities, together with (3.7), (3.16), (3.17), (3.20), (3.26), (3.31) and (3.36), imply that there exist positive constants $c_{i}, i \in\{1,2 \ldots 10\}$, such that

$$
\begin{aligned}
\frac{d}{\mathrm{~d} t} \mathcal{E}_{L M}+\mathcal{D}_{L M} \leqq & \left(\delta+\varepsilon^{2} C_{\delta}\left(Q(\mathcal{E})+1+\mathfrak{G}_{p}\right)+\left(C_{\delta}+\varepsilon^{2}\right) Q(\mathcal{E})\right) \mathcal{D} \\
& +C_{\delta}\left(Q(\mathcal{E})+1+\mathfrak{G}_{p}\right) \mathfrak{H}_{p}\left(\varepsilon^{2}+\mathcal{E}\right)
\end{aligned}
$$

where we denote

$$
\begin{aligned}
\mathcal{E}_{L M}= & \mathcal{E}_{L M}(t):=\frac{c_{1}}{2}\left\|\rho^{1 / 2} \varepsilon \psi_{t}^{h}\right\|_{L^{2}}^{2}+\frac{c_{1} c_{s}^{2}}{2 \rho_{0}}\left\|\xi_{t}\right\|_{L^{2}}^{2}+\frac{c_{2}}{2}\left\|\rho^{1 / 2} \nabla_{h}^{2} \psi^{h}\right\|_{L^{2}}^{2} \\
& +\frac{c_{2} c_{s}^{2}}{2 \rho_{0}}\left\|\varepsilon^{-1} \nabla_{h}^{2} \xi\right\|_{L^{2}}^{2}+c_{3}\left\|\rho^{1 / 2} \psi_{z z}^{h}\right\|_{L^{2}}^{2}+c_{4}\left\|\rho^{1 / 2} \nabla_{h} \psi_{z}^{h}\right\|_{L^{2}}^{2} \\
& +\frac{c_{5}}{2}\left\|\rho^{1 / 2} \nabla_{h} \psi^{h}\right\|_{L^{2}}^{2}+\frac{c_{5} c_{s}^{2}}{2 \rho_{0}}\left\|\varepsilon^{-1} \nabla_{h} \xi\right\|_{L^{2}}^{2}+c_{6}\left\|\rho^{1 / 2} \psi_{z}^{h}\right\|_{L^{2}}^{2} \\
& +\frac{c_{7}}{2}\left\|\rho^{1 / 2} \psi^{h}\right\|_{L^{2}}^{2}+\frac{c_{7} c_{s}^{2}}{2 \rho_{0}}\left\|\varepsilon^{-1} \xi\right\|_{L^{2}}^{2}, \\
\mathcal{D}_{L M}= & \mathcal{D}_{L M}(t):=c_{1}\left\|\varepsilon \nabla \psi_{t}^{h}\right\|_{L^{2}}^{2}+c_{2}\left\|\nabla \nabla_{h}^{2} \psi^{h}\right\|_{L^{2}}^{2}+c_{3}\left\|\nabla \psi_{z z}^{h}\right\|_{L^{2}}^{2} \\
& +c_{4}\left\|\nabla \nabla_{h} \psi_{z}^{h}\right\|_{L^{2}}^{2}+c_{5}\left\|\nabla \nabla_{h} \psi^{h}\right\|_{L^{2}}^{2}+c_{6}\left\|\nabla \psi_{z}^{h}\right\|_{L^{2}}^{2} \\
& +c_{7}\left\|\nabla \psi^{h}\right\|_{L^{2}}^{2}+c_{8}\left\|\xi_{t}\right\|_{L^{2}}^{2}+c_{9}\left\|\varepsilon^{-1} \nabla_{h} \xi\right\|_{H^{1}}^{2}+C_{10}\left\|\varepsilon \rho \psi_{t}^{h}\right\|_{L^{2}}^{2} .
\end{aligned}
$$

Under the assumption (3.2), it is easy to check that

$$
\mathcal{E} \lesssim \mathcal{E}_{L M} \lesssim \mathcal{E}, \mathcal{D} \lesssim \mathcal{D}_{L M} \lesssim \mathcal{D}
$$

where $\mathcal{E}=\mathcal{E}(t)$ and $\mathcal{D}=\mathcal{D}(t)$ are defined in (2.2) and (2.3), respectively. Therefore (3.38) can be written, after choosing $\delta$ small enough, as

$$
\begin{aligned}
\frac{d}{\mathrm{~d} t} \mathcal{E}_{L M}+\mathcal{D}_{L M} \leqq & \left(\varepsilon^{2} C\left(Q(\mathcal{E})+1+\mathfrak{G}_{p}\right)+\left(1+\varepsilon^{2}\right) Q(\mathcal{E})\right) \mathcal{D}_{L M} \\
& +C\left(Q(\mathcal{E})+1+\mathfrak{G}_{p}\right) \mathfrak{H}_{p}\left(\varepsilon^{2}+\mathcal{E}_{L M}\right) .
\end{aligned}
$$


Then after applying Grönwall's inequality to (3.42), one concludes that

$$
\begin{aligned}
& \sup _{0 \leqq t \leqq T} \mathcal{E}_{L M}(t)+\int_{0}^{T} \mathcal{D}_{L M}(t) \mathrm{d} t \lesssim e^{C \int_{0}^{T}\left(Q(\mathcal{E})+1+\mathfrak{G}_{p}\right) \mathfrak{H}_{p} \mathrm{~d} t} \\
& \times\left\{\varepsilon^{2}+\mathcal{E}_{L M}(0)+\int_{0}^{T}\left[\left[\varepsilon^{2} C\left(Q(\mathcal{E})+1+\mathfrak{G}_{p}\right)\right.\right.\right. \\
& \left.\left.\left.+\left(1+\varepsilon^{2}\right) Q(\mathcal{E})\right] \mathcal{D}_{L M}\right] \mathrm{~d} t\right\} .
\end{aligned}
$$

Under the assumptions of Proposition 1, this completes the proof of (3.3).

\section{Low Mach Number Limit}

In this section, we will establish the asymptotic behavior of $\left(\xi, \psi^{h}\right)=\left(\xi^{\varepsilon}, \psi^{\varepsilon, h}\right)$ as $\varepsilon \rightarrow 0^{+}$. In particular, we prove Theorem 1.2 in this section.

First, as a consequence of Theorem 1.1, we have the following:

Corollary 1. Under the same assumptions of Theorem 1.1, consider any integer $s \geqq 3$. Then (3.1) holds true for $v_{p, \text { in }} \in H^{s}\left(\Omega_{h} \times 2 \mathbb{T}\right)$, with the compatibility conditions as in (1.15).

Proof. Directly from the conclusion of Theorem 1.1, for $s \geqq 3$ and $v_{p, \text { in }} \in H^{s}(\Omega)$ as stated in the theorem, one has

$$
\begin{aligned}
& \sup _{0 \leqq t<\infty}\left(\left\|v_{p}(t)\right\|_{H^{3}}+\left\|v_{p, t}(t)\right\|_{L^{2}}\right)+\int_{0}^{\infty}\left(\left\|v_{p}(t)\right\|_{H^{3}}^{2}+\left\|v_{p, t}(t)\right\|_{H^{1}}^{2}\right. \\
& \left.\quad+\left\|v_{p}(t)\right\|_{H^{2}}\right) \mathrm{d} t \leqq C_{p, i n, 3}+1,
\end{aligned}
$$

where we have used the Gagliardo-Nirenberg interpolation inequality

$$
\begin{aligned}
\int_{0}^{\infty}\left\|v_{p}(t)\right\|_{H^{2}} \mathrm{~d} t & \lesssim \int_{0}^{\infty}\left\|v_{p}(t)\right\|_{L^{2}}^{1 / 3}\left\|v_{p}(t)\right\|_{H^{3}}^{2 / 3} \mathrm{~d} t \\
& \lesssim \int_{0}^{\infty} e^{-\frac{c}{3} t} \mathrm{~d} t \times C_{p, i n, 3}^{1 / 2} \leqq C_{p, i n, 3}+1 .
\end{aligned}
$$

Moreover, applying the Minkowski and Hölder inequalities to the expression of $w_{p}$ as in (1.11) yields

$$
\begin{aligned}
\sup _{0 \leqq t<\infty}\left\|w_{p}(t)\right\|_{H^{1}} \leqq \sup _{0 \leqq t<\infty}\left\|v_{p}(t)\right\|_{H^{2}} \leqq C_{p, i n, 2}+1, \\
\int_{0}^{\infty}\left\|w_{p}(t)\right\|_{H^{2}}^{2} \mathrm{~d} t \leqq \int_{0}^{\infty}\left\|v_{p}(t)\right\|_{H^{3}}^{2} \mathrm{~d} t \leqq C_{p, i n, 2} .
\end{aligned}
$$

What is left is to estimate

$$
\left\|\rho_{1}\right\|_{H^{2}},\left\|\rho_{1, t}\right\|_{L^{2}}, \int_{0}^{\infty}\left(\left\|\rho_{1, t t}\right\|_{L^{2}}^{2}+\left\|\rho_{1, t}\right\|_{H^{1}}^{2}+\left\|\rho_{1}\right\|_{H^{2}}^{2}\right) \mathrm{d} t .
$$


To do this, we write down the elliptic problem for $\rho_{1}$, which is obtained by taking average over the $z$-variable and then taking $\operatorname{div}_{h}$ in $(\mathrm{PE})_{2}$, as follows

$$
-c_{s}^{2} \Delta_{h} \rho_{1}=\rho_{0} \int_{0}^{1} \operatorname{div}_{h}\left(\operatorname{div}_{h}\left(v_{p} \otimes v_{p}\right)\right) \mathrm{d} z \text { in } \Omega_{h}, \int_{\Omega_{h}} \rho_{1} \mathrm{~d} x \mathrm{~d} y=0 .
$$

Then the $L^{p}$ estimate of the Riesz transform implies, that together with the Minkowski, Hölder and Sobolev embedding inequalities,

$$
\left\|\rho_{1}\right\|_{H^{2}} \leqq\left|\rho_{1}\right|_{H^{2}} \lesssim\left\|\left|v_{p}\right|^{2}\right\|_{H^{2}} \lesssim\left\|v_{p}\right\|_{H^{2}}^{2}
$$

Consequently, for $s \geqq 2$,

$$
\begin{aligned}
& \sup _{0 \leqq t<\infty}\left\|\rho_{1}(t)\right\|_{H^{2}}+\int_{0}^{\infty}\left\|\rho_{1}(t)\right\|_{H^{2}}^{2} \mathrm{~d} t \lesssim \sup _{0 \leqq t<\infty}\left\|v_{p}(t)\right\|_{H^{2}}^{2} \\
& \quad+\sup _{0 \leqq t<\infty}\left\|v_{p}(t)\right\|_{H^{2}}^{2} \times \int_{0}^{\infty}\left\|v_{p}(t)\right\|_{H^{2}}^{2} \mathrm{~d} t \leqq\left(1+C_{p, i n, 1}\right) C_{p, i n, 2} .
\end{aligned}
$$

Furthermore, after taking time derivatives of (4.1), we have the following elliptic problems:

$$
\begin{aligned}
& \left\{\begin{array}{l}
-c_{s}^{2} \Delta_{h} \rho_{1, t}=2 \rho_{0} \int_{0}^{1} \operatorname{div}_{h}\left(\operatorname{div}_{h}\left(v_{p} \otimes v_{p, t}\right)\right) \mathrm{d} z \text { in } \Omega_{h}, \\
\int_{\Omega_{h}} \rho_{1, t} \mathrm{~d} x \mathrm{~d} y=0 ;
\end{array}\right. \\
& \left\{\begin{array}{c}
-c_{s}^{2} \Delta_{h} \rho_{1, t t}=2 \rho_{0} \int_{0}^{1} \operatorname{div}_{h}\left(\operatorname{div}_{h}\left(v_{p} \otimes v_{p, t t}+v_{p, t} \otimes v_{p, t}\right)\right) \mathrm{d} z \\
\text { in } \Omega_{h}, \quad \int_{\Omega_{h}} \rho_{1, t t} \mathrm{~d} x \mathrm{~d} y=0 .
\end{array}\right.
\end{aligned}
$$

Thus similarly, one has, for $s \geqq 3$,

$$
\begin{aligned}
& \left\|\rho_{1, t}\right\|_{L^{2}} \lesssim\left\|v_{p}\right\|_{H^{2}}\left\|v_{p, t}\right\|_{L^{2}} \leqq C_{p, i n, 2}, \\
& \int_{0}^{\infty}\left\|\rho_{1, t}\right\|_{H^{1}}^{2} \mathrm{~d} t \lesssim \sup _{0 \leqq t<\infty}\left\|v_{p}\right\|_{H^{2}}^{2} \int_{0}^{\infty}\left\|v_{p, t}\right\|_{H^{1}}^{2} \mathrm{~d} t \leqq C_{p, i n, 2}^{2}, \\
& \int_{0}^{\infty}\left\|\rho_{1, t t}\right\|_{L^{2}}^{2} \lesssim \sup _{0 \leqq t<\infty}\left\|v_{p}\right\|_{H^{2}}^{2} \times \int_{0}^{\infty}\left\|v_{p, t t}\right\|_{L^{2}}^{2} \mathrm{~d} t+\sup _{0 \leqq t<\infty}\left\|v_{p, t}\right\|_{H^{1}}^{2} \\
& \quad \times \int_{0}^{\infty}\left\|v_{p, t}\right\|_{H^{1}}^{2} \leqq C_{p, i n, 2} \int_{0}^{\infty}\left\|v_{p, t t}\right\|_{L^{2}}^{2} \mathrm{~d} t+C_{p, i n, 3}^{2} .
\end{aligned}
$$

On the other hand, after taking time derivative of $(\mathrm{PE})_{2}$, we have the identity

$$
\begin{aligned}
\rho_{0} v_{p, t t}= & -\rho_{0}\left(v_{p} \cdot \nabla_{h} v_{p}+w_{p} \partial_{z} v_{p}\right)_{t}-c_{s}^{2} \nabla_{h} \rho_{1, t} \\
& +\mu \Delta_{h} v_{p, t}+\lambda \nabla_{h} \operatorname{div}_{h} v_{p, t}+\partial_{z z} v_{p, t} .
\end{aligned}
$$

Therefore, directly one has,

$$
\left\|v_{p, t t}\right\|_{L^{2}} \lesssim\left\|\rho_{1, t}\right\|_{H^{1}}+\left\|v_{p, t}\right\|_{H^{2}}+\left\|v_{p}\right\|_{H^{3}}\left\|v_{p, t}\right\|_{H^{1}},
$$


where we have applied the Minkowski, Sobolev embedding and Hölder inequalities, and the following inequalities as the consequence of (1.11):

$$
\left\|w_{p}\right\|_{H^{2}} \leqq\left\|v_{p}\right\|_{H^{3}},\left\|w_{p, t}\right\|_{L^{2}} \leqq\left\|v_{p, t}\right\|_{H^{1}}
$$

Consequently, one concludes that, for $s \geqq 3$,

$$
\begin{aligned}
\int_{0}^{\infty}\left\|v_{p, t t}\right\|_{L^{2}}^{2} \mathrm{~d} t \lesssim & \int_{0}^{\infty}\left\|\rho_{1, t}\right\|_{H^{1}}^{2} \mathrm{~d} t+\left(1+\sup _{0 \leqq t<\infty}\left\|v_{p}\right\|_{H^{3}}^{2}\right) \\
& \times \int_{0}^{\infty}\left\|v_{p, t}\right\|_{H^{2}}^{2} \mathrm{~d} t \lesssim C_{p, i n, 2}^{2}+\left(1+C_{p, i n, 3}\right) C_{p, i n, 3},
\end{aligned}
$$

where we have substituted inequality $(4.5)_{2}$. Thus, $(4.5)_{3}$ yields

$$
\int_{0}^{\infty}\left\|\rho_{1, t t}\right\|_{L^{2}}^{2} \lesssim C_{p, i n, 3}^{2}+C_{p, i n, 2}\left(C_{p, i n, 2}^{2}+\left(1+C_{p, i n, 3}\right) C_{p, i n, 3}\right)<\infty
$$

This completes the proof.

Now, given $v_{p, \text { in }} \in H^{s}\left(\Omega_{h} \times 2 \mathbb{T}\right)$, for any integer $s \geqq 3$, which is even in the $z$-variable and satisfies the compatibility conditions (1.15), one can apply the conclusion of Proposition 1 to establish the global bound of the perturbation energy $\mathcal{E}$, provided it is initially small. This is done through a continuity argument. We state first the proposition concerning the local well-posedness of solutions to system (1.8) with $\mathcal{E}_{\text {in }}$ small enough.

Proposition 2. Let $v_{p}$ be the solution to system (PE), as stated in Theorem 1.1 with initial data $v_{p, \text { in }} \in H^{s}\left(\Omega_{h} \times 2 \mathbb{T}\right)$, for an integer $s \geqq 3$. Consider the initial data $\left(\xi_{i n}, \psi_{i n}^{h}\right) \in H^{2}\left(\Omega_{h} \times 2 \mathbb{T}\right) \times H^{2}\left(\Omega_{h} \times 2 \mathbb{T}\right)$ as in (1.12) and satisfying the compatibility condition (1.13). There is a positive constant $\bar{\varepsilon} \in(0,1)$, small enough, and a positive time $T_{\bar{\varepsilon}} \in(0, \infty)$, such that if $\varepsilon \in(0, \bar{\varepsilon})$ and $\mathcal{E}_{\text {in }} \leqq \bar{\varepsilon}$, there exists a unique strong solution $\left(\xi^{\varepsilon}, \psi^{\varepsilon, h}\right) \in L^{\infty}\left(0, T_{\bar{\varepsilon}} ; H^{2}\left(\Omega_{h} \times 2 \mathbb{T}\right)\right)$, with $\psi^{\varepsilon, z}$ as in (1.10), to system (1.8) in the time interval $\left[0, T_{\bar{\varepsilon}}\right]$. The existence time $T_{\bar{\varepsilon}}$ depends only on $\bar{\varepsilon}$ and $\left\|v_{p, \text { in }}\right\|_{H^{3}}$ and is independent of $\varepsilon$. Here $\mathcal{E}_{\text {in }}$ is as in (1.14). Moreover, $\partial_{t} \xi^{\varepsilon}, \partial_{t} \psi^{\varepsilon, h} \in L^{\infty}\left(0, T_{\bar{\varepsilon}} ; L^{2}\left(\Omega_{h} \times 2 \mathbb{T}\right)\right), \rho \in\left(\frac{1}{2} \rho_{0}, 2 \rho_{0}\right)$ in $\Omega \times\left[0, T_{\bar{\varepsilon}}\right]$, and there is a constant $C^{\prime \prime}>0$, independent of $\varepsilon$ such that

$$
\sup _{0 \leqq t \leqq T_{\bar{\varepsilon}}} \mathcal{E}(t) \leqq C^{\prime \prime} \mathcal{E}_{i n}
$$

where $\mathcal{E}(t)$ is as in (2.2).

The proof of Proposition 2 can be done via a fixed point argument similar to that in our previous work [50] and it is omitted here.

Now we are ready to establish the proof of Theorem 1.2 
Proof of Theorem 1.2. Consider $s \geqq 3$ and $\varepsilon \in(0, \bar{\varepsilon})$ with $\bar{\varepsilon} \in(0,1)$ as given in Proposition 2. Let the initial data $\left(\xi_{i n}, \psi_{i n}^{h}\right) \in H^{2}\left(\Omega_{h}\right) \times H^{2}\left(\Omega_{h} \times 2 \mathbb{T}\right)$ satisfy (1.12), the compatibility conditions (1.13), and $\mathcal{E}_{\text {in }} \leqq \varepsilon^{2}$, where $\mathcal{E}_{\text {in }}$ is as in (1.14). Then $\mathcal{E}_{i n} \leqq \bar{\varepsilon}$, and there is a strong solution to system (1.8) as stated by Proposition 2 in the time interval $\left[0, T_{\bar{\varepsilon}}\right]$, for some $T_{\bar{\varepsilon}} \in(0, \infty)$, independent of $\varepsilon$. The strong solution satisfies

$$
\begin{aligned}
& \rho \in\left(\frac{1}{2} \rho_{0}, 2 \rho_{0}\right) \text { in }\left(\Omega_{h} \times 2 \mathbb{T}\right) \times\left[0, T_{\bar{\varepsilon}}\right], \\
& \text { and } \sup _{0 \leqq t \leqq T_{\bar{\varepsilon}}} \mathcal{E}(t) \leqq C^{\prime \prime} \mathcal{E}_{i n} \leqq C^{\prime \prime} \varepsilon^{2} .
\end{aligned}
$$

Such estimates, together with Theorem 1.1 and Corollary 1 , imply that the assumptions in Proposition 1 hold true in the time interval $\left[0, T_{\bar{\varepsilon}}\right]$. Therefore applying (3.3) yields

$$
\begin{aligned}
\sup _{0 \leqq t \leqq T_{\bar{\varepsilon}}} \mathcal{E}(t)+\int_{0}^{T_{\bar{\varepsilon}}} \mathcal{D}(t) \mathrm{d} t \leqq & C^{\prime} e^{C^{\prime}+Q\left(C^{\prime \prime} \varepsilon^{2}\right)}\left\{\varepsilon^{2}+\varepsilon^{2}\right. \\
& \left.+\left(\varepsilon^{2}+\left(\varepsilon^{2}+1\right) Q\left(C^{\prime \prime} \varepsilon^{2}\right)\right) \int_{0}^{T_{\bar{\varepsilon}}} \mathcal{D}(t) \mathrm{d} t\right\} \\
\leqq & \left.2 C^{\prime} e^{2 C^{\prime}} \varepsilon^{2}+\frac{1}{2} \int_{0}^{T_{\bar{\varepsilon}}} \mathcal{D}(t) \mathrm{d} t\right\}
\end{aligned}
$$

provided $\varepsilon \in\left(0, \varepsilon_{1}\right) \subset(0, \bar{\varepsilon})$, where $\varepsilon_{1}$ is small enough such that $Q\left(C^{\prime \prime} \varepsilon_{1}^{2}\right) \leqq C^{\prime}$ and $C^{\prime} e^{2 C^{\prime}}\left(\varepsilon_{1}^{2}+\left(\varepsilon_{1}^{2}+1\right) Q\left(C^{\prime \prime} \varepsilon_{1}^{2}\right)\right) \leqq 1 / 2$. This inequality yields that

$$
\sup _{0 \leqq t \leqq T_{\bar{\varepsilon}}} \mathcal{E}(t)+\int_{0}^{T_{\bar{\varepsilon}}} \mathcal{D}(t) \mathrm{d} t \leqq C^{\prime \prime \prime} \varepsilon^{2}<\bar{\varepsilon}
$$

where $C^{\prime \prime \prime}=4 C^{\prime} e^{2 C^{\prime}}$, and provided $\varepsilon_{1}$ is small such that $C^{\prime \prime \prime} \varepsilon_{1}^{2}<\bar{\varepsilon}$. In particular, $\mathcal{E}\left(T_{\bar{\varepsilon}}\right) \leqq \bar{\varepsilon}$. We apply Proposition 2 again in the time interval $\left[T_{\bar{\varepsilon}}, 2 T_{\bar{\varepsilon}}\right]$, which states that there exists a strong solution satisfying

$$
\begin{aligned}
& \rho \in\left(\frac{1}{2} \rho_{0}, 2 \rho_{0}\right) \text { in }\left(\Omega_{h} \times 2 \mathbb{T}\right) \times\left[T_{\bar{\varepsilon}}, 2 T_{\bar{\varepsilon}}\right], \\
& \text { and } \sup _{T_{\bar{\varepsilon}} \leqq t \leqq 2 T_{\bar{\varepsilon}}} \mathcal{E}(t) \leqq C^{\prime \prime} \mathcal{E}\left(T_{\bar{\varepsilon}}\right) \leqq C^{\prime \prime} C^{\prime \prime \prime} \varepsilon^{2} .
\end{aligned}
$$

Together with (4.6), this implies that

$$
\sup _{0 \leqq t \leqq 2 T_{\bar{\varepsilon}}} \mathcal{E}(t) \leqq C^{\prime \prime \prime \prime \prime} \varepsilon^{2} \text { with } C^{\prime \prime \prime \prime}=\max \left\{C^{\prime \prime}, C^{\prime \prime} C^{\prime \prime \prime}\right\} .
$$

Consequently, Proposition 1 applies. In particular, (3.3) yields

$$
\begin{aligned}
\sup _{0 \leqq t \leqq 2 T_{\bar{\varepsilon}}} \mathcal{E}(t)+\int_{0}^{2 T_{\bar{\varepsilon}}} \mathcal{D}(t) \mathrm{d} t \leqq & C^{\prime} e^{C^{\prime}+Q\left(C^{\prime \prime \prime \prime} \varepsilon^{2}\right)}\left\{\varepsilon^{2}+\varepsilon^{2}\right. \\
& \left.+\left(\varepsilon^{2}+\left(\varepsilon^{2}+1\right) Q\left(C^{\prime \prime \prime \prime} \varepsilon^{2}\right)\right) \int_{0}^{2 T_{\bar{\varepsilon}}} \mathcal{D}(t) \mathrm{d} t\right\} .
\end{aligned}
$$


As above, this implies

$$
\sup _{0 \leqq t \leqq 2 T_{\bar{\varepsilon}}} \mathcal{E}(t)+\int_{0}^{2 T_{\bar{\varepsilon}}} \mathcal{D}(t) \mathrm{d} t \leqq C^{\prime \prime \prime} \varepsilon^{2}<\bar{\varepsilon}
$$

provided that $\varepsilon \in\left(0, \varepsilon_{2}\right) \subset\left(0, \varepsilon_{1}\right) \subset(0, \bar{\varepsilon})$, for $\varepsilon_{2}$ small enough such that $Q\left(C^{\prime \prime \prime \prime} \varepsilon_{2}^{2}\right) \leqq Q\left(C^{\prime \prime} \varepsilon_{1}^{2}\right)$. Then inductively, without needing to determine the smallness of $\varepsilon$ again, the arguments from (4.6) to (4.7) hold true for $T_{\bar{\varepsilon}}, 2 T_{\bar{\varepsilon}}$ replaced by $n T_{\bar{\varepsilon}},(n+1) T_{\bar{\varepsilon}}, n \geqq 2$, respectively. In particular, (4.7) holds true for $2 T_{\bar{\varepsilon}}$ replaced by $(n+1) T_{\bar{\varepsilon}}$. Recall that $T_{\bar{\varepsilon}}$ is independent of $\varepsilon$. This concludes the proof of (1.18). (1.19) is a direct consequence of (1.18), (1.3), (1.9), (1.11) and the fact that $\left\|\rho_{1}\right\|_{H^{2}}<\infty$ as in (4.2). Therefore let $\varepsilon_{0}=\varepsilon_{2}$, and we complete the proof of Theorem 1.2.

\section{Global Regularity Estimates of the Solution to the Primitive Equations}

Let us recall the primitive equations (PE) first. We shorten the notations $v=$ $v_{p}, w=w_{p}$ in this section. Recall that

$$
\begin{cases}\operatorname{div}_{h} v+\partial_{z} w=0 & \text { in } \Omega_{h} \times 2 \mathbb{T}, \\ \rho_{0}\left(\partial_{t} v+v \cdot \nabla_{h} v+w \partial_{z} v\right)+\nabla_{h}\left(c_{s}^{2} \rho_{1}\right)=\mu \Delta_{h} v & \\ +\lambda \nabla_{h} \operatorname{div}_{h} v+\partial_{z z} v & \text { in } \Omega_{h} \times 2 \mathbb{T}, \\ \partial_{z}\left(c_{s}^{2} \rho_{1}\right)=0 & \text { in } \Omega_{h} \times 2 \mathbb{T},\end{cases}
$$

where $\mu>0, \lambda>\frac{1}{3} \mu>0$. Here the symmetry (SYM-PE) and the side condition (1.1) are imposed. We will make further assumptions on the viscous coefficients later.

In this section, we will study the global regularity of the solution $\left(\rho_{1}, v, w\right)$ to (PE) with initial data $v_{p, i n} \in H^{s}\left(\Omega_{h} \times 2 \mathbb{T}\right)$ for arbitrary integer $s \in\{1,2,3 \ldots\}$, with $v_{p, i n}$ being even in the $z$-variable and satisfying the compatible conditions as stated in Theorem 1.1.

We will show the following proposition:

Proposition 3. For $0<\lambda<4 \mu<12 \lambda$, suppose (PE) is complemented with initial data $v_{p, i n} \in H^{1}\left(\Omega_{h} \times 2 \mathbb{T}\right)$ as above. Then the unique solution $v_{p}$ to the primitive equations (PE) satisfies the estimates as stated in Theorem 1.1.

As in [41], we focus on the a priori estimates below. In fact, the local-in-time regularity in Sobolev and analytic function spaces has been studied in [56], and therefore following a continuity argument, one can check the validity of the proof below. 


\section{Basic Energy Estimate}

Take the $L^{2}$-inner product of $(\mathrm{PE})_{2}$ with $v$. We have

$$
\frac{d}{\mathrm{~d} t}\left\{\frac{\rho_{0}}{2}\|v\|_{L^{2}}^{2}\right\}+\mu\left\|\nabla_{h} v\right\|_{L^{2}}^{2}+\lambda\left\|\operatorname{div}_{h} v\right\|_{L^{2}}^{2}+\left\|\partial_{z} v\right\|_{L^{2}}^{2}=0 .
$$

Integrating the above equation in the time variable yields

$$
\begin{aligned}
& \sup _{0 \leqq t<\infty}\left\{\frac{\rho_{0}}{2}\|v(t)\|_{L^{2}}^{2}\right\}+\int_{0}^{\infty}\left(\mu\left\|\nabla_{h} v(t)\right\|_{L^{2}}^{2}+\lambda\left\|\operatorname{div}_{h} v(t)\right\|_{L^{2}}^{2}\right. \\
& \left.\quad+\left\|\partial_{z} v(t)\right\|_{L^{2}}^{2}\right) \mathrm{d} t=\frac{\rho_{0}}{2}\left\|v_{p, i n}\right\|_{L^{2}}^{2} .
\end{aligned}
$$

Moreover, under the assumption (1.2), after applying the Poincaré inequality in (5.1), we have the inequality

$$
\frac{d}{\mathrm{~d} t}\|v\|_{L^{2}}^{2}+c\|v\|_{L^{2}}^{2} \leqq 0
$$

for some positive constant $c$. Thus one can derive from above that

$$
\|v(t)\|_{L^{2}}^{2} \lesssim e^{-c t}\left\|v_{p, i n}\right\|_{L^{2}}^{2}
$$

for all $t \in[0, \infty)$.

\section{$H^{1}$ Estimate}

After applying $\partial_{z}$ to $(\mathrm{PE})_{2}$, we write down the following equation:

$$
\begin{aligned}
\rho_{0}\left(\partial_{t} v_{z}+v \cdot \nabla_{h} v_{z}+w \partial_{z} v_{z}\right)= & \mu \Delta_{h} v_{z}+\lambda \nabla_{h} \operatorname{div}_{h} v_{z}+\partial_{z z} v_{z} \\
& -\rho_{0}\left(v_{z} \cdot \nabla_{h} v+w_{z} \partial_{z} v\right) .
\end{aligned}
$$

Then take the $L^{2}$ inner product of (5.4) with $v_{z}$. It follows, after substituting (1.11), that

$$
\begin{aligned}
& \frac{d}{\mathrm{~d} t}\left\{\frac{\rho_{0}}{2}\left\|v_{z}\right\|_{L^{2}}^{2}\right\}+\mu\left\|\nabla_{h} v_{z}\right\|_{L^{2}}^{2}+\lambda\left\|\operatorname{div}_{h} v_{z}\right\|_{L^{2}}^{2}+\left\|\partial_{z} v_{z}\right\|_{L^{2}}^{2} \\
& \quad=-\rho_{0} \int\left(v_{z} \cdot \nabla_{h} v\right) \cdot v_{z} \mathrm{~d} \vec{x}+\rho_{0} \int \operatorname{div}_{h} v\left(\partial_{z} v \cdot v_{z}\right) \mathrm{d} \vec{x}=: L_{1}+L_{2} .
\end{aligned}
$$

After applying integration by parts, one will have

$$
\begin{aligned}
& L_{1}=\rho_{0} \int\left(v_{z} \cdot \nabla_{h} v_{z}\right) \cdot v \mathrm{~d} \vec{x}+\rho_{0} \int\left(v \cdot v_{z}\right) \operatorname{div}_{h} v_{z} \mathrm{~d} \vec{x}, \\
& L_{2}=-2 \rho_{0} \int\left(v \cdot \nabla_{h} v_{z}\right) \cdot v_{z} \mathrm{~d} \vec{x} .
\end{aligned}
$$

Therefore, let $p, q$ be some positive constants, to be determined later, satisfying

$$
\frac{1}{p}+\frac{1}{q}=\frac{1}{2}, \quad 2<p<6 \text { (equivalently } q>3 \text { ). }
$$


After applying Hölder's, the Gagliardo-Nirenberg interpolation and Young's inequalities, one has

$$
\begin{aligned}
L_{1}+L_{2} \lesssim & \left\|v_{z}\right\|_{L^{p}}\left\|\nabla_{h} v_{z}\right\|_{L^{2}}\|v\|_{L^{q}} \lesssim\left\|v_{z}\right\|_{L^{2}}^{3 / p-1 / 2}\left\|\nabla v_{z}\right\|_{L^{2}}^{3 / 2-3 / p} \\
& \times\left\|\nabla_{h} v_{z}\right\|_{L^{2}}\|v\|_{L^{q}} \lesssim \delta\left\|\nabla v_{z}\right\|_{L^{2}}^{2}+C_{\delta}\left\|v_{z}\right\|_{L^{2}}^{2}\|v\|_{L^{q}}^{4 p /(6-p)} \\
= & \delta\left\|\nabla v_{z}\right\|_{L^{2}}^{2}+C_{\delta}\left\|v_{z}\right\|_{L^{2}}^{2}\|v\|_{L^{q}}^{2 q /(q-3)} .
\end{aligned}
$$

Hence, after choosing $\delta>0$, sufficiently small, in the above estimate, we have

$$
\begin{aligned}
& \frac{d}{\mathrm{~d} t}\left\{\frac{\rho_{0}}{2}\left\|v_{z}\right\|_{L^{2}}^{2}\right\}+\frac{\mu}{2}\left\|\nabla_{h} v_{z}\right\|_{L^{2}}^{2}+\lambda\left\|\operatorname{div}_{h} v_{z}\right\|_{L^{2}}^{2}+\frac{1}{2}\left\|\partial_{z} v_{z}\right\|_{L^{2}}^{2} \\
& \quad \lesssim\left\|v_{z}\right\|_{L^{2}}^{2}\|v\|_{L^{q}}^{\frac{2 q}{q-3}} .
\end{aligned}
$$

Integrating the above inequality in the time variable yields

$$
\begin{aligned}
& \sup _{0 \leqq t<\infty}\left\|v_{z}(t)\right\|_{L^{2}}^{2}+\int_{0}^{\infty}\left\|\nabla v_{z}(t)\right\|_{L^{2}}^{2} \mathrm{~d} t \lesssim \sup _{0 \leqq t<\infty}\|v(t)\|_{L^{q}}^{\frac{2 q}{q-3}} \\
& \quad \times \int_{0}^{\infty}\|\nabla v(t)\|_{L^{2}}^{2} \mathrm{~d} t+\left\|v_{p, i n, z}\right\|_{L^{2}}^{2} \lesssim \sup _{0 \leqq t<\infty}\|v(t)\|_{L^{q}}^{\frac{2 q}{q-3}} \\
& \quad \times\left\|v_{p, i n}\right\|_{L^{2}}^{2}+\left\|v_{p, i n, z}\right\|_{L^{2}}^{2}
\end{aligned}
$$

for $q \in(3, \infty)$, where we have substituted (5.2).

On the other hand, after applying $\partial_{h}$ to $(\mathrm{PE})_{2}$, one gets the equation

$$
\begin{aligned}
& \rho_{0}\left(\partial_{t} v_{h}+v \cdot \nabla_{h} v_{h}+w \partial_{z} v_{h}\right)+\nabla_{h}\left(c_{s}^{2} \rho_{1, h}\right)=\mu \Delta_{h} v_{h} \\
& +\lambda \nabla_{h} \operatorname{div}_{h} v_{h}+\partial_{z z} v_{h}-\rho_{0}\left(v_{h} \cdot \nabla_{h} v+w_{h} \partial_{z} v\right) .
\end{aligned}
$$

Then after taking $L^{2}$-inner product of (5.8) with $v_{h}$, we have

$$
\begin{aligned}
& \frac{d}{\mathrm{~d} t}\left\{\frac{\rho_{0}}{2}\left\|v_{h}\right\|_{L^{2}}^{2}\right\}+\mu\left\|\nabla_{h} v_{h}\right\|_{L^{2}}^{2}+\lambda\left\|\operatorname{div}_{h} v_{h}\right\|_{L^{2}}^{2}+\left\|\partial_{z} v_{h}\right\|_{L^{2}}^{2} \\
& \quad=-\rho_{0} \int\left(v_{h} \cdot \nabla_{h} v\right) \cdot v_{h} \mathrm{~d} \vec{x}-\rho_{0} \int w_{h}\left(\partial_{z} v \cdot v_{h}\right) \mathrm{d} \vec{x}=: L_{3}+L_{4} .
\end{aligned}
$$

Now we estimate the terms on the right-hand side of the above equality. As before, let $q>3, \frac{1}{p}+\frac{1}{q}=\frac{1}{2}$. After applying integration by parts and the Hölder, GagliardoNirenberg interpolation and Young inequalities, one has

$$
\begin{aligned}
L_{3} \lesssim & \left\|\nabla_{h} v\right\|_{L^{p}}\left\|\nabla_{h}^{2} v\right\|_{L^{2}}\|v\|_{L^{q}} \lesssim \delta\left\|\nabla \nabla_{h} v\right\|_{L^{2}}^{2} \\
& +C_{\delta}\left\|\nabla_{h} v\right\|_{L^{2}}^{2}\|v\|_{L^{q}}^{2 q /(q-3)} .
\end{aligned}
$$

On the other hand, after substituting (1.11) in the term $L_{4}$ and applying the Minkowski, Hölder's, the Gagliardo-Nirenberg interpolation and Young's inequalities, one has

$$
L_{4}=\rho_{0} \int\left(\int_{0}^{z} \operatorname{div}_{h} v_{h} \mathrm{~d} z\right) \times\left(\partial_{z} v \cdot v_{h}\right) \mathrm{d} \vec{x} \lesssim \int_{0}^{1}\left|\nabla_{h}^{2} v\right|_{L^{2}} \mathrm{~d} z
$$




$$
\begin{aligned}
& \times \int_{0}^{1}\left|v_{z}\right|_{L^{4}}\left|v_{h}\right|_{L^{4}} \mathrm{~d} z \lesssim\left\|\nabla_{h}^{2} v\right\|_{L^{2}}\left\|v_{z}\right\|_{L^{2}}^{1 / 2}\left\|\nabla_{h} v_{z}\right\|_{L^{2}}^{1 / 2} \\
& \times\left\|v_{h}\right\|_{L^{2}}^{1 / 2}\left\|\nabla_{h} v_{h}\right\|_{L^{2}}^{1 / 2} \lesssim \delta\left\|\nabla \nabla_{h} v\right\|_{L^{2}}^{2}+C_{\delta}\left\|v_{z}\right\|_{L^{2}}^{2} \\
& \times\left\|\nabla_{h} v_{z}\right\|_{L^{2}}^{2}\left\|v_{h}\right\|_{L^{2}}^{2} .
\end{aligned}
$$

Similarly, take the $L^{2}$ inner product of $(\mathrm{PE})_{2}$ with $v_{t}$. One has,

$$
\begin{aligned}
& \frac{d}{\mathrm{~d} t}\left\{\frac{\mu}{2}\left\|\nabla_{h} v\right\|_{L^{2}}^{2}+\frac{\lambda}{2}\left\|\operatorname{div}_{h} v\right\|_{L^{2}}^{2}+\frac{1}{2}\left\|\partial_{z} v\right\|_{L^{2}}^{2}\right\}+\rho_{0}\left\|\partial_{t} v\right\|_{L^{2}}^{2} \\
& \quad=-\rho_{0} \int\left(v \cdot \nabla_{h} v\right) \cdot v_{t} \mathrm{~d} \vec{x}-\rho_{0} \int w\left(\partial_{z} v \cdot v_{t}\right) \mathrm{d} \vec{x}=: L_{5}+L_{6} .
\end{aligned}
$$

As before, applying the Hölder, Minkowski, Gagliardo-Nirenberg interpolation and Young inequalities yield, for $q>3, \frac{1}{p}+\frac{1}{q}=\frac{1}{2}$,

$$
\begin{aligned}
L_{5} \lesssim & \left\|\nabla_{h} v\right\|_{L^{p}}\left\|v_{t}\right\|_{L^{2}}\|v\|_{L^{q}} \lesssim\left\|\nabla_{h} v\right\|_{L^{2}}^{3 / p-1 / 2}\left\|\nabla \nabla_{h} v\right\|_{L^{2}}^{3 / 2-3 / p} \\
& \times\left\|v_{t}\right\|_{L^{2}}\|v\|_{L^{q}} \lesssim \delta\left(\left\|v_{t}\right\|_{L^{2}}^{2}+\left\|\nabla \nabla_{h} v\right\|_{L^{2}}^{2}\right) \\
& +C_{\delta}\left\|\nabla_{h} v\right\|_{L^{2}}^{2}\|v\|_{L^{q}}^{2 q /(q-3)}, \\
L_{6} \lesssim & \delta\left(\left\|v_{t}\right\|_{L^{2}}^{2}+\left\|\nabla_{h}^{2} v\right\|_{L^{2}}^{2}\right)+C_{\delta}\left\|v_{z}\right\|_{L^{2}}^{2}\left\|\nabla_{h} v_{z}\right\|_{L^{2}}^{2}\left\|\nabla_{h} v\right\|_{L^{2}}^{2} .
\end{aligned}
$$

After summing (5.9), (5.10) and the estimates of $L_{3}, L_{4}, L_{5}, L_{6}$ above with sufficiently small $\delta$, one has

$$
\begin{aligned}
& \frac{d}{\mathrm{~d} t}\left\{\frac{\rho_{0}}{2}\left\|v_{h}\right\|_{L^{2}}^{2}+\frac{\mu}{2}\left\|\nabla_{h} v\right\|_{L^{2}}^{2}+\frac{\lambda}{2}\left\|\operatorname{div}_{h} v\right\|_{L^{2}}^{2}+\frac{1}{2}\left\|\partial_{z} v\right\|_{L^{2}}^{2}\right\} \\
& \quad+\frac{\mu}{2}\left\|\nabla_{h} v_{h}\right\|_{L^{2}}^{2}+\lambda\left\|\operatorname{div}_{h} v_{h}\right\|_{L^{2}}^{2}+\frac{1}{2}\left\|\partial_{z} v_{h}\right\|_{L^{2}}^{2}+\frac{\rho_{0}}{2}\left\|\partial_{t} v\right\|_{L^{2}}^{2} \\
& \quad \lesssim\left\|\nabla_{h} v\right\|_{L^{2}}^{2}\|v\|_{L^{q}}^{2 q /(q-3)}+\left\|v_{z}\right\|_{L^{2}}^{2}\left\|\nabla_{h} v_{z}\right\|_{L^{2}}^{2}\left\|\nabla_{h} v\right\|_{L^{2}}^{2} .
\end{aligned}
$$

Then after applying the Grönwall's inequality, it follows that

$$
\begin{aligned}
& \sup _{0 \leqq t<\infty}\|\nabla v(t)\|_{L^{2}}^{2}+\int_{0}^{\infty}\left(\left\|\nabla v_{h}(t)\right\|_{L^{2}}^{2}+\left\|\partial_{t} v(t)\right\|_{L^{2}}^{2}\right) \mathrm{d} t \\
& \lesssim e^{C \int_{0}^{\infty}\left\|v_{z}(t)\right\|_{L^{2}}^{2}\left\|\nabla_{h} v_{z}(t)\right\|_{L^{2}}^{2} \mathrm{~d} t}\left(\left\|\nabla v_{p, i n}\right\|_{L^{2}}^{2}\right. \\
& \left.\quad+\int_{0}^{\infty}\left\|\nabla_{h} v(t)\right\|_{L^{2}}^{2} \mathrm{~d} t \times \sup _{0 \leqq t<\infty}\|v(t)\|_{L^{q}}^{\frac{2 q}{q-3}}\right), \\
& \lesssim e^{C\left(\sup _{0 \leqq t<\infty}\|v(t)\|_{L^{q}}^{\frac{4 q}{q-3}} \times\left\|v_{p, i n}\right\|_{L^{2}}^{4}+\left\|v_{p, i n, z}\right\|_{L^{2}}^{4}\right)}\left(\left\|\nabla v_{p, i n}\right\|_{L^{2}}^{2}\right. \\
& \left.\quad+\left\|v_{p, i n}\right\|_{L^{2}}^{2} \times \sup _{0 \leqq t<\infty}\|v(t)\|_{L^{q}}^{\frac{2 q}{q-3}}\right)
\end{aligned}
$$

for some positive constant $C$ and $q \in(3, \infty)$, where we have substituted (5.2) and (5.7). 


\section{$L^{q}$ Estimate}

We take the $L^{2}$-inner product of (PE) 2 with $|v|^{q-2} v$. It follows that

$$
\begin{aligned}
& \frac{d}{\mathrm{~d} t}\left\{\frac{\rho_{0}}{q}\|v\|_{L^{q}}^{q}\right\}+\mu \int\left(|v|^{q-2}\left|\nabla_{h} v\right|^{2}+(q-2)|v|^{q-2}\left|\nabla_{h}\right| v||^{2}\right) \mathrm{d} \vec{x} \\
& \quad+\lambda \int|v|^{q-2}\left|\operatorname{div}_{h} v\right|^{2} \mathrm{~d} \vec{x}+\int\left(|v|^{q-2}\left|\partial_{z} v\right|^{2}\right. \\
& \left.\quad+(q-2)|v|^{q-2}\left|\partial_{z}\right| v||^{2}\right) \mathrm{d} \vec{x}=\int c_{s}^{2} \rho_{1} \operatorname{div}_{h}\left(|v|^{q-2} v\right) \mathrm{d} \vec{x} \\
& \quad-\lambda(q-2) \int|v|^{q-3}\left(v \cdot \nabla_{h}|v|\right) \operatorname{div}_{h} v \mathrm{~d} \vec{x}=: L_{7}+L_{8} .
\end{aligned}
$$

By using the Cauchy-Schwarz inequality, it holds that

$$
\begin{aligned}
L_{8} \leqq & \lambda(q-2) \int|v|^{q-2}\left|\nabla_{h}\right| v||\left|\operatorname{div}_{h} v\right| \mathrm{d} \vec{x} \leqq \lambda \int|v|^{q-2}\left|\operatorname{div}_{h} v\right|^{2} \mathrm{~d} \vec{x} \\
& +\frac{\lambda(q-2)^{2}}{4} \int|v|^{q-2}\left|\nabla_{h}\right| v||^{2} \mathrm{~d} \vec{x}
\end{aligned}
$$

Therefore, (5.12) implies

$$
\begin{aligned}
\frac{d}{\mathrm{~d} t}\left\{\frac{\rho_{0}}{q}\|v\|_{L^{q}}^{q}\right\}+\mu\left\||v|^{\frac{q}{2}-1} \nabla_{h} v\right\|_{L^{2}}^{2}+\left\||v|^{\frac{q}{2}-1} \partial_{z} v\right\|_{L^{2}}^{2} \\
\quad \lesssim \frac{d}{\mathrm{~d} t}\left\{\frac{\rho_{0}}{q}\|v\|_{L^{q}}^{q}\right\}+\int\left(\mu|v|^{q-2}\left|\nabla_{h} v\right|^{2}\right. \\
\left.\quad+\left.\left(\mu(q-2)-\frac{\lambda(q-2)^{2}}{4}\right)|v|^{q-2}\left|\nabla_{h}\right| v\right|^{2}\right) \mathrm{d} \vec{x} \\
\quad+\int\left(|v|^{q-2}\left|\partial_{z} v\right|^{2}+\left.(q-2)|v|^{q-2}\left|\partial_{z}\right| v\right|^{2}\right) \mathrm{d} \vec{x} \leqq L_{7},
\end{aligned}
$$

provided

$$
q-2 \geqq 0 \text { and } \mu-\frac{\lambda(q-2)}{4} \geqq 0, \text { or equivalently, } 2 \leqq q \leqq \frac{4 \mu}{\lambda}+2 .
$$

In order to estimate $L_{7}$, we first derive an estimate for the "pressure" $\rho_{1}$. Recall the elliptic problem (4.1):

$$
-c_{s}^{2} \Delta_{h} \rho_{1}=\rho_{0} \int_{0}^{1} \operatorname{div}_{h}\left(\operatorname{div}_{h}(v \otimes v)\right) \mathrm{d} z \text { in } \Omega_{h}, \text { with } \int_{\Omega_{h}} \rho_{1} \mathrm{~d} x \mathrm{~d} y=0
$$


Now we consider the $L^{p_{1}}$ estimate of $\rho_{1}$. In fact, as a consequence of the $L^{p}$ estimate of the Riesz transform, one has

$$
\begin{aligned}
\left\|\rho_{1}\right\|_{L^{p_{1}}} & =\left.\left.\left|\rho_{1}\right|_{L^{p_{1}}} \lesssim \int_{0}^{1}|| v\right|^{2}\right|_{L^{p_{1}}} \mathrm{~d} z=\int_{0}^{1}|v|_{L^{2 p_{1}}}^{2} \mathrm{~d} z \lesssim \int_{0}^{1}|v|_{L^{4}}|v|_{L^{4 p_{1} /\left(4-p_{1}\right)}} \mathrm{d} z \\
& =\left.\left.\int_{0}^{1}|v|_{L^{4}}|| v\right|^{\frac{q}{2}}\right|^{\frac{2}{q}} \frac{8 p_{1}}{L^{\left(4-p_{1}\right)}} \mathrm{d} z \\
& \left.\left.\left.\left.\lesssim \int_{0}^{1}|v|_{L^{2}}^{\frac{1}{2}}\left|\nabla_{h} v\right|_{L^{2}}^{\frac{1}{2}}|| v\right|^{\frac{q}{2}}\right|_{L^{2}} ^{\frac{2}{p_{1}}-\frac{1}{2}}\left|\nabla_{h}\right| v\right|^{\frac{q}{2}}\right|_{L^{2}} ^{\frac{1}{2}+\frac{2}{q}-\frac{2}{p_{1}}} \mathrm{~d} z \\
& \lesssim\|v\|_{L^{2}}^{\frac{1}{2}}\left\|\nabla_{h} v\right\|_{L^{2}}^{\frac{1}{2}}\|v\|_{L^{q}}^{\frac{q}{p_{1}}-\frac{q}{4}}\left\||v|^{\frac{q}{2}-1} \nabla_{h} v\right\|_{L^{2}}^{\frac{1}{2}+\frac{2}{q}-\frac{2}{p_{1}}},
\end{aligned}
$$

provided

$$
p_{1}>2, \quad \frac{1}{4}<\frac{1}{p_{1}}<\frac{1}{q}+\frac{1}{4}, q \geqq 2,
$$

where we have applied the Minkowski, Hölder and Gagliardo-Nirenberg interpolation inequalities. Let $q_{1}>0$ be such that

$$
\frac{1}{p_{1}}+\frac{1}{q_{1}}=\frac{1}{2} \text {. }
$$

Then we have, after applying the Minkowski, Hölder and Gagliardo-Nirenberg interpolation inequalities,

$$
\begin{aligned}
& L_{7} \lesssim\left.\left.\int\left|\rho_{1}\right||| v\right|^{\frac{q}{2}-1}\left|\nabla_{h} v\right||| v\right|^{\frac{q}{2}-1} \mathrm{~d} \vec{x} \lesssim\left|\rho_{1}\right|_{L^{p_{1}}} \\
& \times\left.\left.\left.\left.\int_{0}^{1}|| v\right|^{\frac{q}{2}-1} \nabla_{h} v\right|_{L^{2}}|| v\right|^{\frac{q}{2}-1}\right|_{L^{q_{1}}} \mathrm{~d} z=\left\|\rho_{1}\right\|_{L^{p_{1}}} \\
& \times \int_{0}^{1}\left\|\left.\left.\left.\left.v\right|^{\frac{q}{2}-1} \nabla_{h} v\right|_{L^{2}}|| v\right|^{\frac{q}{2}}\right|_{L^{\frac{q-2}{q}}} ^{\frac{q_{1}(q-2)}{q}} \mathrm{~d} z \lesssim\right\| \rho_{1} \|_{L^{p_{1}}} \\
& \times \int_{0}^{1} \|\left.\left.\left.\left.\left.\left. v\right|^{\frac{q}{2}-1} \nabla_{h} v\right|_{L^{2}}|| v\right|^{\frac{q}{2}}\right|_{L^{2}} ^{\frac{2}{q_{1}}}\left|\nabla_{h}\right| v\right|^{\frac{q}{2}}\right|_{L^{2}} ^{1-\frac{2}{q}-\frac{2}{q_{1}}} \mathrm{~d} z \\
& \lesssim\left\|\rho_{1}\right\| L_{L^{p_{1}}}\left\||v|^{\frac{q}{2}-1} \nabla_{h} v\right\|_{L^{2}}\|v\|_{L^{q}}^{\frac{q}{2}-\frac{q}{p_{1}}}\left\||v|^{\frac{q}{2}-1} \nabla_{h} v\right\|_{L^{2}}^{\frac{2}{p_{1}}-\frac{2}{q}} \\
& \lesssim\|v\|_{L^{2}}^{1 / 2}\left\|\nabla_{h} v\right\|_{L^{2}}^{1 / 2}\left\||v|^{\frac{q}{2}-1} \nabla_{h} v\right\|_{L^{2}}^{\frac{3}{2}}\|v\|_{L^{q}}^{\frac{q}{4}} \\
& \lesssim \delta\left\||v|^{\frac{q}{2}-1} \nabla_{h} v\right\|_{L^{2}}^{2}+C_{\delta}\|v\|_{L^{2}}^{2}\left\|\nabla_{h} v\right\|_{L^{2}}^{2}\|v\|_{L^{q}}^{q},
\end{aligned}
$$

provided

$$
0<\frac{1}{q_{1}}<\frac{1}{2}-\frac{1}{q}, \text { or equivalently, } \frac{1}{q}<\frac{1}{p_{1}} \text { and } q>2,
$$

where we have substituted (5.15) in the second but last inequality. Therefore after combining (5.14), (5.16), (5.18), for $q$ satisfying

$$
2<q \leqq \frac{4 \mu}{\lambda}+2
$$


we conclude from (5.13) and (5.17) that

$$
\begin{aligned}
& \frac{d}{\mathrm{~d} t}\left\{\frac{\rho_{0}}{q}\|v\|_{L^{q}}^{q}\right\}+\frac{\mu}{2}\left\||v|^{\frac{q}{2}-1} \nabla_{h} v\right\|_{L^{2}}^{2}+\left\||v|^{\frac{q}{2}-1} \partial_{z} v\right\|_{L^{2}}^{2} \\
& \quad \lesssim\|v\|_{L^{2}}^{2}\left\|\nabla_{h} v\right\|_{L^{2}}^{2}\|v\|_{L^{q}}^{q},
\end{aligned}
$$

after choosing $\delta$ sufficiently small above. Applying Grönwall's inequality to the above inequality implies, for $2<q \leqq \frac{4 \mu}{\lambda}+2$,

$$
\begin{aligned}
& \sup _{0 \leqq t<T}\|v(t)\|_{L^{q}}^{q}+\int_{0}^{\infty}\left\||v|^{\frac{q}{2}-1} \nabla v(t)\right\|_{L^{2}}^{2} \mathrm{~d} t \\
& \quad \lesssim C_{q} e^{C \int_{0}^{\infty}\|v(t)\|_{L^{2}}^{2}\left\|\nabla_{h} v(t)\right\|_{L^{2}}^{2} \mathrm{~d} t}\left\|v_{p, i n}\right\|_{L^{q}}^{q} \\
& \quad \lesssim C_{q} e^{C}\left\|v_{p, i n}\right\|_{L^{2}}^{4}\left\|v_{p, i n}\right\|_{L^{q}}^{q},
\end{aligned}
$$

for some positive constant $C$ and $C_{q}$ depending on $q$.

Therefore, after summing up the inequalities (5.2), (5.7), (5.11), (5.20), for $\lambda<4 \mu<12 \lambda$, one will have

$$
\begin{aligned}
& \sup _{0 \leqq t<\infty}\|v(t)\|_{H^{1}}^{2}+\int_{0}^{\infty}\left(\|\nabla v(t)\|_{H^{1}}^{2}+\left\|\partial_{t} v(t)\right\|_{L^{2}}^{2}\right) \mathrm{d} t \\
& \quad \leqq C_{p, i n}\left(\left\|v_{p, i n}\right\|_{H^{1}},\left\|v_{p, i n}\right\|_{L^{q}}\right)
\end{aligned}
$$

for some positive constant $C_{p, \text { in }}$ depending on $\left\|v_{p, \text { in }}\right\|_{H^{1}},\left\|v_{p, i n}\right\|_{L^{q}}$ with

$$
3<q \leqq \frac{4 \mu}{\lambda}+2, \quad \frac{4 \mu}{\lambda} \in(1,12)
$$

In particular, it suffices to take

$$
q=\left\{\begin{array}{ll}
4 & \text { if } \frac{4 \mu}{\lambda} \in[4,12), \\
\frac{4 \mu}{\lambda}+2 & \text { if } \frac{4 \mu}{\lambda} \in(1,4),
\end{array} \quad \text { such that } q \in[2,6]\right.
$$

and therefore

$$
C_{p, i n}\left(\left\|v_{p, i n}\right\|_{H^{1}},\left\|v_{p, i n}\right\|_{L^{q}}\right)=C_{p, i n}\left(\left\|v_{p, i n}\right\|_{H^{1}}\right)
$$

depends only on $\left\|v_{p, i n}\right\|_{H^{1}}$ by noticing that $\left\|v_{p, \text { in }}\right\|_{L^{q}} \lesssim\left\|v_{p, i n}\right\|_{H^{1}}$ in this case. The estimate of $\partial_{t} v_{p}$ follows directly from $(5.21)$ and $(\mathrm{PE})_{2}$. 


\section{$H^{s}$ Estimates}

Next, we will show the global regularity of the solution $v$ to system (PE) with more regular initial data $v_{p, i n}$. That is, we complement (PE) with the initial data $v_{p, \text { in }} \in H^{s}(\Omega)$, with $s \geqq 2$. In fact, we will use the mathematical induction principle to show (1.17). Notice, the case when $s=1$, i.e., (1.16), has been shown in (5.21).

First, for integer $s \geqq 1$, it is assumed that (1.17) holds true. Our goal is to show that the same estimate is also true for $s$ replaced by $s+1$. In order to do so, we apply $\partial^{s+1}$ to $(\mathrm{PE})_{2}$ with $\partial \in\left\{\partial_{x}, \partial_{y}, \partial_{z}\right\}$ and denote the $k$-order derivative by ${ }_{k}:=\partial^{k}$. for any $k \in\{0,1,2 \ldots s+1\}$. Then we have the following equation:

$$
\begin{aligned}
& \rho_{0}\left(\partial_{t} v_{s+1}+v \cdot \nabla_{h} v_{s+1}+w \partial_{z} v_{s+1}\right)+\nabla_{h}\left(c_{s}^{2} \rho_{1, s+1}\right) \\
& =\mu \Delta_{h} v_{s+1}+\lambda \nabla_{h} \operatorname{div}_{h} v_{s+1}+\partial_{z z} v_{s+1} \\
& \quad-\rho_{0}\left(\partial^{s+1}\left(v \cdot \nabla_{h} v\right)-v \cdot \nabla_{h} v_{s+1}+\partial^{s+1}\left(w \partial_{z} v\right)-w \partial_{z} v_{s+1}\right) .
\end{aligned}
$$

Take the $L^{2}$-inner product of (5.23) with $v_{s+1}$. It follows,

$$
\begin{aligned}
& \frac{d}{\mathrm{~d} t}\left\{\frac{\rho_{0}}{2}\left\|v_{s+1}\right\|_{L^{2}}^{2}\right\}+\mu\left\|\nabla_{h} v_{s+1}\right\|_{L^{2}}^{2}+\lambda\left\|\operatorname{div}_{h} v_{s+1}\right\|_{L^{2}}^{2} \\
& \quad+\left\|\partial_{z} v_{s+1}\right\|_{L^{2}}^{2}=\rho_{0} \int\left(v \cdot \nabla_{h} v_{s+1}-\partial^{s+1}\left(v \cdot \nabla_{h} v\right)\right) \cdot v_{s+1} \mathrm{~d} \vec{x} \\
& \quad+\rho_{0} \int\left(w \partial_{z} v_{s+1}-\partial^{s+1}\left(w \partial_{z} v\right)\right) \cdot v_{s+1} \mathrm{~d} \vec{x}=: K_{1}+K_{2} .
\end{aligned}
$$

We estimate $K_{1}, K_{2}$ on the right-hand side of (5.24). First, notice that $K_{1}, K_{2}$ can be written as

$$
\begin{aligned}
& K_{1}=-\rho_{0} \sum_{i=0}^{s}\left(\begin{array}{c}
s+1 \\
i
\end{array}\right) \int\left(v_{s+1-i} \cdot \nabla_{h} v_{i}\right) \cdot v_{s+1} \mathrm{~d} \vec{x}=: \sum_{i=0}^{s} K_{1, i}, \\
& K_{2}=-\rho_{0} \sum_{i=0}^{s}\left(\begin{array}{c}
s+1 \\
i
\end{array}\right) \int w_{s+1-i} \partial_{z} v_{i} \cdot v_{s+1} \mathrm{~d} \vec{x}=: \sum_{i=0}^{s} K_{2, i} .
\end{aligned}
$$

We consider the estimates of $K_{j, i}$, for $j \in\{1,2\}$ and $i \in\{0,1,2 \ldots s\}$ in three cases:

$$
\left\{\begin{array}{l}
2 \leqq i \leqq s \\
i=1 \\
i=0
\end{array}\right.
$$

In the case when $i \geqq 2$, we have

$$
s+1-i \leqq s-1,3 \leqq 1+i \leqq s+1 .
$$

Therefore, applying the Hölder, Sobolev embedding and Young inequalities implies

$$
\begin{aligned}
K_{1, i} & \lesssim\left\|v_{s+1-i}\right\|_{L^{3}}\left\|\nabla_{h} v_{i}\right\|_{L^{6}}\left\|v_{s+1}\right\|_{L^{2}} \lesssim\|v\|_{H^{s+2-i}}\|v\|_{H^{i+2}}\left\|v_{s+1}\right\|_{L^{2}} \\
& \lesssim\|v\|_{H^{s}}\|v\|_{H^{s+2}}\left\|v_{s+1}\right\|_{L^{2}} \lesssim \delta\|v\|_{H^{s+2}}^{2}+C_{\delta}\|v\|_{H^{s}}^{2}\left\|v_{s+1}\right\|_{L^{2}}^{2}
\end{aligned}
$$


Similarly, we apply the Minkowski, Hölder, Sobolev embedding and Young inequalities to estimate $K_{2, i}$. On the one hand, if $w_{s+1-i}=\partial^{s+1-i} w=\partial_{h}^{s+1-i} w$, we have, thanks to (1.11),

$$
\begin{aligned}
K_{2, i} & =\rho_{0}\left(\begin{array}{c}
s+1 \\
i
\end{array}\right) \int\left(\partial_{h}^{s+1-i}\left(\int_{0}^{z} \operatorname{div}_{h} v \mathrm{~d} z^{\prime}\right) \times\left(\partial_{z} v_{i} \cdot v_{s+1}\right)\right) \mathrm{d} \vec{x} \\
& \lesssim \int_{0}^{1}\left|v_{s+2-i}\right|_{L^{2}}^{1 / 2}\left|v_{s+3-i}\right|_{L^{2}}^{1 / 2} \mathrm{~d} z^{\prime} \times \int_{0}^{1}\left|\partial_{z} v_{i}\right|_{L^{2}}^{1 / 2}\left|\partial_{z} v_{i+1}\right|_{L^{2}}^{1 / 2}\left|v_{s+1}\right|_{L^{2}} \mathrm{~d} z \\
& \lesssim\|v\| H_{H^{s}}^{1 / 2}\|v\|_{H^{s+1}}\|v\|_{H^{s+2}}^{1 / 2}\left\|v_{s+1}\right\|_{2} \lesssim \delta\|v\|_{H^{s+2}}^{2} \\
& +C_{\delta}\|v\|_{H^{s+1}}^{2}\left\|v_{s+1}\right\|_{L^{2}}^{2} .
\end{aligned}
$$

On the other hand, if $w_{s+1-i}=\partial_{z} w_{s-i}$, we have

$$
\begin{aligned}
K_{2, i} & =\rho_{0}\left(\begin{array}{c}
s+1 \\
i
\end{array}\right) \int \operatorname{div}_{h} v_{s-i} \partial_{z} v_{i} \cdot v_{s+1} \mathrm{~d} \vec{x} \\
& \lesssim \delta\|v\|_{H^{s+2}}^{2}+C_{\delta}\|v\|_{H^{s}}^{2}\left\|v_{s+1}\right\|_{L^{2}}^{2} .
\end{aligned}
$$

In the case when $i=1$, direct application of the Hölder, Sobolev embedding and Young inequalities yields

$$
K_{1,1} \lesssim \delta\|v\|_{H^{s+2}}^{2}+C_{\delta}\|v\|_{H^{2}}^{2}\|v\|_{H^{s+1}}^{2}
$$

Meanwhile, to estimate $K_{2,1}$, we will again apply the Minkowski, Hölder, Sobolev embedding and Young inequalities. If $w_{s}=\partial_{h}^{s} w$, we have, after substituting (1.11),

$$
\begin{aligned}
K_{2,1} & =\rho_{0}\left(\begin{array}{c}
s+1 \\
1
\end{array}\right) \int\left(\left(\int_{0}^{z} \operatorname{div}_{h} \partial_{h}^{s} v \mathrm{~d} z^{\prime}\right) \times\left(\partial_{z} v_{1} \cdot v_{s+1}\right)\right) \mathrm{d} \vec{x} \\
& \lesssim \int_{0}^{1}\left|\operatorname{div}_{h} v_{s}\right|_{L^{2}}^{1 / 2}\left|\nabla_{h} \operatorname{div}_{h} v_{s}\right|_{L^{2}}^{1 / 2} \mathrm{~d} z^{\prime} \times \int_{0}^{1}\left|\partial_{z} v_{1}\right|_{L^{2}}\left|v_{s+1}\right|_{L^{2}}^{1 / 2}\left|\nabla_{h} v_{s+1}\right|_{L^{2}}^{1 / 2} \mathrm{~d} z \\
& \lesssim\|v\|\left\|_{H^{s+1}}\right\| v\left\|_{H^{s+2}}\right\| v\left\|_{H^{2}} \lesssim \delta\right\| v\left\|_{H^{s+2}}^{2}+C_{\delta}\right\| v\left\|_{H^{2}}^{2}\right\| v \|_{H^{s+1}}^{2} .
\end{aligned}
$$

If $w_{s}=\partial_{z} w_{s-1}$, we have

$$
K_{2,1} \lesssim \delta\|v\|_{H^{s+2}}^{2}+C_{\delta}\|v\|_{H^{2}}^{2}\|v\|_{H^{s+1}}^{2} .
$$

Finally, in the case when $i=0$, we apply the Hölder, Sobolev embedding and Young inequalities to get

$$
K_{1,0} \lesssim \delta\|v\|_{H^{s+2}}^{2}+C_{\delta}\|v\|_{H^{2}}^{2}\left\|v_{s+1}\right\|_{L^{2}}^{2} .
$$

When $w_{s+1}=\partial_{h}^{s+1} w$, applying the Minkowski, Hölder, Sobolev embedding and Young inequalities yields, after substituting (1.11),

$$
\begin{aligned}
K_{2,0} & =\rho_{0}\left(\begin{array}{c}
s+1 \\
0
\end{array}\right) \int\left(\partial_{h}^{s+1}\left(\int_{0}^{z} \operatorname{div}_{h} v \mathrm{~d} z^{\prime}\right) \times\left(\partial_{z} v \cdot v_{s+1}\right)\right) \mathrm{d} \vec{x} \\
& \lesssim \int_{0}^{1}\left|v_{s+2}\right|_{L^{2}} \mathrm{~d} z^{\prime} \times \int_{0}^{1}\left|\partial_{z} v\right|_{L^{4}}\left|v_{s+1}\right|_{L^{4}} \mathrm{~d} z
\end{aligned}
$$




$$
\begin{aligned}
& \lesssim\|v\|_{H^{s+2}}^{3 / 2}\left\|v_{s+1}\right\|_{L^{2}}^{1 / 2}\|v\|_{H^{1}}^{1 / 2}\|v\|_{H^{2}}^{1 / 2} \\
& \lesssim \delta\|v\|_{H^{s+2}}^{2}+C_{\delta}\|v\|_{H^{1}}^{2}\|v\|_{H^{2}}^{2}\left\|v_{s+1}\right\|_{L^{2}}^{2}
\end{aligned}
$$

When $w_{s+1}=\partial_{z} w_{s}$, we have, after substituting (1.11),

$$
\begin{aligned}
K_{2,0} & =\rho_{0}\left(\begin{array}{c}
s+1 \\
0
\end{array}\right) \int \operatorname{div}_{h} v_{s} \partial_{z} v \cdot v_{s+1} \mathrm{~d} \vec{x} \lesssim\left\|v_{s+1}\right\|_{L^{6}}\|\nabla v\|_{L^{3}}\left\|v_{s+1}\right\|_{L^{2}} \\
& \lesssim \delta\|v\|_{H^{s+2}}^{2}+C_{\delta}\|v\|_{H^{2}}^{2}\left\|v_{s+1}\right\|_{L^{2}}^{2} .
\end{aligned}
$$

From the above estimates, one can conclude from (5.24) that for any integer $s \geqq 1$

$$
\begin{aligned}
& \frac{d}{\mathrm{~d} t}\left\{\frac{\rho_{0}}{2}\left\|v_{s+1}\right\|_{L^{2}}^{2}\right\}+\mu\left\|\nabla_{h} v_{s+1}\right\|_{L^{2}}^{2}+\lambda\left\|\operatorname{div}_{h} v_{s+1}\right\|_{L^{2}}^{2} \\
& \quad+\left\|\partial_{z} v_{s+1}\right\|_{L^{2}}^{2} \lesssim \delta\left\|\nabla v_{s+1}\right\|_{2}^{2}+\left(\delta+C_{\delta}\|v\|_{H^{s}}^{2}\right)\|v\|_{H^{s+1}}^{2} \\
& \quad+C_{\delta}\left(\|v\|_{H^{s+1}}^{2}+\|v\|_{H^{1}}^{2}\|v\|_{H^{2}}^{2}\right)\left\|v_{s+1}\right\|_{L^{2}}^{2} .
\end{aligned}
$$

Here we have used the notation $\left\|v_{s+1}\right\|_{L^{2}}^{2}$ to denote $\sum_{\partial \in\left\{\partial_{x}, \partial_{y}, \partial_{z}\right\}}\left\|\partial^{s+1} v\right\|_{L^{2}}^{2}$. Then after taking $\delta>0$ small enough and applying Grönwall's inequality, together with the inequalities (1.17) and (5.21), we have

$$
\begin{aligned}
\sup _{0 \leqq t<\infty}\|v(t)\|_{H^{s+1}}^{2}+\int_{0}^{\infty}\|v(t)\|_{H^{s+2}}^{2} \mathrm{~d} t \\
\quad \lesssim e^{C \int_{0}^{\infty}\left(\|v(t)\|_{H^{s+1}}^{2}+\|v(t)\|_{H^{1}}^{2}\|v(t)\|_{H^{2}}^{2}\right) \mathrm{d} t}\left(\left\|v_{p, i n}\right\|_{H^{s+1}}^{2}\right. \\
\left.\quad+\int_{0}^{\infty}\left(1+\|v(t)\|_{H^{s}}^{2}\right)\|v(t)\|_{H^{s+1}}^{2} \mathrm{~d} t\right) \lesssim e^{C_{p, i n, s}+C_{p, i n, 1}^{2}} \\
\quad \times\left(\left\|v_{p, i n}\right\|_{H^{s+1}}^{2}+C_{p, i n, s}+C_{p, i n, s}^{2}\right)
\end{aligned}
$$

where $C_{p, \text { in }, 1}=C_{p, \text { in }}\left(\left\|v_{p, \text { in }}\right\|_{H^{1}}\right)$ is as in (5.22).

On the other hand, after replacing $s+1$ by $s$ in (5.23), we have the identity

$$
\begin{aligned}
\rho_{0} \partial_{t} v_{s}= & -\nabla_{h}\left(c_{s}^{2} \rho_{1, s}\right)+\mu \Delta_{h} v_{s}+\lambda \nabla_{h} \operatorname{div}_{h} v_{s}+\partial_{z z} v_{s} \\
& -\rho_{0} \partial^{s}\left(v \cdot \nabla_{h} v\right)-\rho_{0} \partial^{s}\left(w \partial_{z} v\right)
\end{aligned}
$$

After taking the $L^{2}$-inner product of (5.27) with $\partial_{t} v_{s}$ and noticing the fact that

$$
-\int \nabla_{h}\left(c_{s}^{2} \rho_{1, s}\right) \cdot \partial_{t} v_{s} \mathrm{~d} \vec{x}=\int c_{s}^{2} \rho_{1, s} \operatorname{div}_{h} \partial_{t} v_{s} \mathrm{~d} \vec{x}=0,
$$

this implies

$$
\left\|\partial_{t} v_{s}\right\|_{L^{2}}^{2} \lesssim\left(1+\|v\|_{H^{s+1}}^{2}\right)\|v\|_{H^{s+2}}^{2},
$$

where we have applied that since $s \geqq 1$,

$$
\left\|v_{i} \cdot \nabla_{h} v_{s-i}\right\|_{L^{2}}^{2} \lesssim\left\|v_{i}\right\|_{L^{3}}^{2}\left\|\nabla_{h} v_{s-i}\right\|_{L^{6}}^{2} \lesssim\|v\|_{H^{i+1}}^{2}\|v\|_{H^{s+2-i}}^{2}
$$




$$
\begin{aligned}
& \lesssim\|v\|_{H^{s+1}}^{2}\|v\|_{H^{s+2}}^{2}, \\
\left\|w_{i} \partial_{z} v_{s-i}\right\|_{L^{2}}^{2} & \lesssim\|w\|_{H^{i+1}}^{2}\|v\|_{H^{s+2-i}}^{2} \lesssim\|v\|_{H^{i+2}}^{2}\|v\|_{H^{s+2-i}}^{2} \\
& \lesssim\|v\|_{H^{s+1}}^{2}\|v\|_{H^{s+2}}^{2},
\end{aligned}
$$

due to the fact that from (1.11),

$$
\|w\|_{H^{i+1}}=\left\|\int_{0}^{z} \operatorname{div}_{h} v \mathrm{~d} z^{\prime}\right\|_{H^{i+1}} \lesssim\|v\|_{H^{i+2}} .
$$

Here we have applied the Minkowski, Hölder and Sobolev embedding inequalities. Similarly, taking $s=s-1$ in (5.28) yields

$$
\left\|\partial_{t} v_{s-1}\right\|_{L^{2}}^{2} \lesssim\left(1+\|v\|_{H^{s}}^{2}\right)\|v\|_{H^{s+1}}^{2} .
$$

Integrating (5.26) in the time variable, together with (5.28), (5.29), implies (1.17) with $s$ replaced by $s+1$. This finishes the mathematical induction. Hence, this concludes the proof of Proposition 3.

Acknowledgements. Open Access funding provided by Projekt DEAL. This work was supported in part by the Einstein Stiftung/Foundation-Berlin, through the Einstein Visiting Fellow Program, and by the John Simon Guggenheim Memorial Foundation.

Open Access This article is licensed under a Creative Commons Attribution 4.0 International License, which permits use, sharing, adaptation, distribution and reproduction in any medium or format, as long as you give appropriate credit to the original author(s) and the source, provide a link to the Creative Commons licence, and indicate if changes were made. The images or other third party material in this article are included in the article's Creative Commons licence, unless indicated otherwise in a credit line to the material. If material is not included in the article's Creative Commons licence and your intended use is not permitted by statutory regulation or exceeds the permitted use, you will need to obtain permission directly from the copyright holder. To view a copy of this licence, visit http://creativecommons.org/ licenses/by/4.0\%.

Publisher's Note Springer Nature remains neutral with regard to jurisdictional claims in published maps and institutional affiliations.

\section{References}

1. Alazard, T.: Low Mach number flows and combustion. SIAM J. Math. Anal. 38(4), 1186-1213, 2006. https://doi.org/10.1137/050644100

2. Alazard, T.: Low Mach number limit of the full Navier-Stokes equations. Arch. Ration. Mech. Anal. 180(1), 1-73, 2006. https://doi.org/10.1007/s00205-005-0393-2

3. AzÉRAd, P., GuIlléN, F.: Mathematical justification of the hydrostatic approximation in the primitive equations of geophysical fluid dynamics. SIAM J. Math. Anal. 33(4), 847-859, 2001. https://doi.org/10.1137/S0036141000375962

4. Bahouri, H., Chemin, J.-Y., Danchin, R.: Fourier analysis and nonlinear partial differential equations. Vol. 343. Springer Science \& Business Media, 2011. https://doi. org/10.1007/978-3-642-16830-7

5. Brenier, Y.: Homogeneous hydrostatic flows with convex velocity profiles. Nonlinearity 12(3), 495-512, 1999. https://doi.org/10.1088/0951-7715/12/3/004 
6. Cao, C., Ibrahim, S., Nakanishi, K., Titi, E.S.: Finite-time blowup for the inviscid primitive equations of oceanic and atmospheric dynamics. Commun. Math. Phys. 337(2), 473-482, 2015. https://doi.org/10.1007/s00220-015-2365-1

7. Cao, C., Li, J., Titi, E.S.: Global well-posedness of strong solutions to the 3D primitive equations with horizontal eddy diffusivity. J. Differ. Equ. 257(11), 4108-4132, 2014. https://doi.org/10.1016/j.jde.2014.08.003

8. CaO, C., Li, J., Titi, E.S.: Local and global well-posedness of strong solutions to the 3D primitive equations with vertical eddy diffusivity. Arch. Ration. Mech. Anal. 214(1), 35-76, 2014. https://doi.org/10.1007/s00205-014-0752-y

9. CaO, C., Li, J., Tiтi, E.S.: Global well-posedness of the three-dimensional primitive equations with only horizontal viscosity and diffusion. Commun. Pure Appl. Math. 69(8), 1492-1531, 2016. https://doi.org/10.1002/cpa.21576

10. CaO, C., LI, J., Titi, E.S.: Strong solutions to the $3 \mathrm{D}$ primitive equations with only horizontal dissipation: near $H^{1}$ initial data. J. Funct. Anal. 272(11), 4606-4641, 2017. https://doi.org/10.1016/j.jfa.2017.01.018

11. CaO, C., LI, J., Titi, E.S.: Global well-posedness of the 3D primitive equations with horizontal viscosity and vertical diffusivity. arXiv:1703.02512, 2017

12. CaO, C., Tiтi, E.S.: Global well-posedness of the three-dimensional viscous primitive equations of large scale ocean and atmosphere dynamics. Ann. Math. 166(1), 245-267, 2007. https://doi.org/10.4007/annals.2007.166.245

13. CAO, C., Titi, E.S.: Global well-posedness of the 3D primitive equations with partial vertical turbulence mixing heat diffusion. Commun. Math. Phys. 310(2), 537-568, 2012. https://doi.org/10.1007/s00220-011-1409-4

14. Chemin, J.-Y., Desjardins, B., Gallagher, I., Grenier, E.: Mathematical geophysics: an introduction to rotating fluids and the Navier-Stokes equations. Vol. 32. Oxford University Press on Demand, 2006

15. Zelati, M.C., Huang, A., Kunavica, I., Temam, R., Ziane, M.: The primitive equations of the atmosphere in presence of vapour saturation. Nonlinearity 28(3), 625-668, 2015. https://doi.org/10.1088/0951-7715/28/3/625

16. DANChin, R.: Zero Mach number limit for compressible flows with periodic boundary conditions. Am. J. Math. 124(6), 1153-1219, 2002

17. DANCHIN, R.: Zero Mach number limit in critical spaces for compressible Navier-Stokes equations. Ann. Scient. Éc. Norm. Sup. 35(1), 27-75, 2002

18. Danchin, R.: Low Mach number limit for viscous compressible flows. ESAIM Math. Model. Numer. Anal. 39(3), 459-475, 2005. https://doi.org/10.1051/m2an:2005019

19. Danchin, R., He, L.: The Oberbeck-Boussinesq approximation in critical spaces. Asymptot. Anal. 84(1-2), 61-102, 2013. https://doi.org/10.3233/ASY-131170

20. Desjardins, B., Grenier, E.: Low Mach number limit of viscous compressible flows in the whole space. Proc. R. Soc. A Math. Phys. Eng. Sci. 455(1986), 2271-2279, 1999. https://doi.org/10.1098/rspa.1999.0403

21. Ersoy, M., NGOM, T.: Existence of a global weak solution to compressible primitive equations. C. R. Acad. Sci. Paris Ser. I 350(7-8), 379-382, 2012. https://doi.org/10. 1016/j.crma.2012.04.013

22. Ersoy, M., NGom, T., Sy, M.: Compressible primitive equations: formal derivation and stability of weak solutions. Nonlinearity 24(1), 79-96, 2011. https://doi.org/10.1088/ 0951-7715/24/1/004

23. FeIreIsL, E.: Dynamics of Viscous Compressible Fluids. Oxford Lecture Series in Mathematics and its Applications, 26. Oxford University Press, 2004

24. FeIREISL, E.: Flows of viscous compressible fluids under strong stratification: incompressible limits for long-range potential forces. Math. Model. Methods Appl. Sci. 21(01), 7-27, 2011. https://doi.org/10.1142/S0218202511004964

25. Feireisl, E., Klein, R., Novotný, A., Zatorska, E.: On singular limits arising in the scale analysis of stratified fluid flows. Math. Model. Methods Appl. Sci. 26(03), 419-443, 2016. https://doi.org/10.1142/S021820251650007X 
26. Feireisl, E., Málek, J., Novotný, A., StrašKraba, I.: Anelastic approximation as a singular limit of the compressible Navier-Stokes system. Commun. Partial Differ. Equ. 33(1), 157-176, 2008. https://doi.org/10.1080/03605300601088799

27. Feireisl, E., Novotný, A.: Singular Limits in Thermodynamics of Viscous Fluids. Advances in Mathematical Fluid Mechanics. Springer International Publishing, Cham 2017

28. Feireisl, E., SchonbeK, M.E.: On the Oberbeck-Boussinesq approximation on unbounded domains. In Nonlinear Partial Differ. Equations, pp. 131-168. Springer, Berlin, 2012. https://doi.org/10.1007/978-3-642-25361-4_7

29. Gallagher, I.: Applications of Schochet's methods to parabolic equations. J. Math. Pures Appl. 77(10), 989-1054, 1998. https://doi.org/10.1016/S0021-7824(99)80002-6

30. Gatapov, B.V., Kazhikhov, A.V.: Existence of a global solution to one model problem of atmosphere dynamics. Sib. Math. J. 46(5), 805-812, 2005. https://doi.org/10.1007/ S11202-005-0079-X

31. Gerard-Varet, D., Masmoudi, N., Vicol, V.: Well-posedness of the hydrostatic Navier-Stokes equations. J. Math. Fluid Mech. 14(2), 355-361, 2018

32. Ginibre, J., Velo, G.: Generalized Strichartz inequalities for the wave equation. $J$. Funct. Anal. 133(1), 50-68, 1995. https://doi.org/10.1006/jfan.1995.1119

33. Guillén-GonZÁlez, F.M., Masmoudi, N., RodríGueZ-Bellido, M.Á.: Anisotropic estimates and strong solutions of the primitive equations. Differ. Integral Equ. 14(11), 1381-1408, 2001

34. Hittmeir, S., Klein, R., Li, J., Titi, E.S.: Global well-posedness for passively transported nonlinear moisture dynamics with phase changes. Nonlinearity 30, 3676-3718, 2017. https://doi.org/10.1088/1361-6544/aa82f1

35. Hoff, D.: The zero-mach limit of compressible flows. Commun. Math. Phys. 192, 543554, 1998. https://doi.org/10.1007/s002200050308

36. Changbing, H., Temam, R., Ziane, M.: The primitive equations on the large scale ocean under the small depth hypothesis. Discret. Contin. Dyn. Syst. 9(1), 97-131, 2002. https://doi.org/10.3934/dcds.2003.9.97

37. KeEL, M., TAO, T.: Endpoint Strichartz estimates. Am. J. Math. 120(5), 955-980, 1998

38. Klainerman, S., Majda, A.: Singular limits of quasilinear hyperbolic systems with large parameters and the incompressible limit of compressible fluids. Commun. Pure Appl. Math. 34(4), 481-524, 1981. https://doi.org/10.1002/cpa.3160340405

39. Klainerman, S., Majda, A.: Compressible and incompressible fluids. Commun. Pure Appl. Math. 35(5), 629-651, 1982. https://doi.org/10.1002/cpa.3160350503

40. Kukavica, I., Temam, R., Vicol, V.C., Ziane, M.: Local existence and uniqueness for the hydrostatic Euler equations on a bounded domain. J. Differ. Equ. 250(3), 1719-1746, 2011. https://doi.org/10.1016/j.jde.2010.07.032

41. Li, J., TITI, E.S.: The primitive equations as the small aspect ratio limit of the NavierStokes equations: Rigorous justification of the hydrostatic approximation. J. Math. Pures Appl. 2018. https://doi.org/10.1016/j.matpur.2018.04.006

42. Lindblad, H., Sogge, C.D.: On existence and scattering with minimal regularity for semilinear wave equations. J. Funct. Anal. 130(2), 357-426, 1995. https://doi.org/10. 1006/jfan.1995.1075

43. Lions, J.-L., Temam, R., Wang, S.: Geostrophic asymptotics of the primitive equations of the atmosphere. Topol. Methods Nonlinear Anal. 4, 253-287, 1994

44. Lions, J.-L., Temam, R., Wang, S.: On the equations of the large-scale ocean. Nonlinearity 5(5), 1007-1053, 1992. https://doi.org/10.1088/0951-7715/5/5/002

45. Lions, J.-L., Temam, R., Wang, S.: New formulations of the primitive equations of atmosphere and applications. Nonlinearity 5(2), 237-288, 1992. https://doi.org/10.1088/ 0951-7715/5/2/001

46. Lions, P.-L., Masmoudi, N.: Incompressible limit for a viscous compressible fluid. J. Math. Pures Appl. 77(6), 585-627, 1998. https://doi.org/10.1016/S00217824(98)80139-6 
47. Lions, P.-L.: Mathematical Topics in Fluid Mechanics. Volume 1. Incompressible Models. Oxford Lecture Series in Mathematics and Its Applications, 3. Oxford University Press, 1996.

48. Lions, P.-L.: Mathematical Topics in Fluid Mechanics. Volume 2. Compressible Models. Oxford Lecture Series in Mathematics and Its Applications, Vol 2, No 10. Oxford University Press, 1998.

49. LiU, X., Titi, E.S.: Global existence of weak solutions to compressible primitive equations with degenerate viscosities. SIAM J. Math. Anal. 51(3), 1913-1964, 2019. https:// doi.org/10.1137/18M1211994

50. Liu, X., Tiтi, E.S.: Local well-posedness of strong solutions to the three-dimensional compressible primitive equations. Available at arXiv:1806.09868, 2018.

51. LiU, X., Titi, E.S.: Zero Mach number limit of the compressible primitive equations Part II: Ill-prepared initial data. In preperation.

52. Masmoudi, N.: Incompressible, inviscid limit of the compressible Navier-Stokes system. Ann. Inst. Henri Poincaré Anal. Nonlinéaire 18(2), 199-224, 2001. https://doi.org/ 10.1016/S0294-1449(00)00123-2

53. Masmoudi, N., Wong, T.K.: On the $H^{s}$ theory of hydrostatic Euler equations. Arch. Ration. Mech. Anal. 204(1), 231-271, 2012. https://doi.org/10.1007/s00205-011-04850

54. NovotnÝ, A., Straskraba, I.: Introduction to the mathematical theory of compressible flow. Vol. 27. Oxford University Press on Demand, 2004

55. NovotnÝ, A., RůžičKa, M., ThäTer, G.: Rigorous derivation of the anelastic approximation to the Oberbeck-Boussinesq equations. Asymptot. Anal.75, 93-123, 2011. https://doi.org/10.3233/ASY-2011-1056

56. Petcu, M., Wirosoetisno, D.: Sobolev and Gevrey regularity results for the primitive equations in three space dimensions. Appl. Anal. 84(8), 769-788, 2005. https://doi.org/ $10.1080 / 00036810500130745$

57. Rajagopal, K.R., Ruzicka, M., Srinivasa, A.R.: On the Oberbeck-Boussinesq approximation. Math. Model. Methods Appl. Sci. 06(08), 1157-1167, 1996. https://doi. org/10.1142/S0218202596000481

58. Renardy, M.: Ill-posedness of the hydrostatic Euler and Navier-Stokes equations. Arch. Ration. Mech. Anal. 194(3), 877-886, 2009. https://doi.org/10.1007/s00205-008-02074

59. Lewis, F.: Richardson. Cambridge University Press, Weather Prediction by Numerical Process 2007

60. Sсноснет, S.: Fast singular limits of hyperbolic PDEs. J. Differ. Equ. 114(2), 476-512, 1994. https://doi.org/10.1006/jdeq.1994.1157

61. Schochet, S.: The compressible Euler equations in a bounded domain: existence of solutions and the incompressible limit. Commun. Math. Phys. 104(1), 49-75, 1986. https://doi.org/10.1007/BF01210792

62. Scноснет, S.: Asymptotics for symmetric hyperbolic systems with a large parameter. J. Differ. Equ. 75(1), 1-27, 1988. https://doi.org/10.1016/0022-0396(88)90126-X

63. TANG, T., GAO, H.: On the stability of weak solution for compressible primitive equations. Acta Appl. Math. 140(1), 133-145, 2015. https://doi.org/10.1007/s10440-0149982-0

64. UkaI, S.: The incompressible limit and the initial layer of the compressible Euler equation. J. Math. Kyoto Univ. 26(2), 323-331, 1986

65. WANG, F., Dou, C., JiU, Q.: Global weak solutions to 3D compressible primitive equations with density-dependent viscosity. J. Math. Phys. 61, 021507, 2020. https://doi. org/10.1063/1.5120088

66. Warren, M.: Washington and Claire L. University Science Books, Parkinson. An Introduction to Three-Dimensional Climate Modeling 2005

67. Wong, T.K.: Blowup of solutions of the hydrostatic Euler equations. Proc. Am. Math. Soc. 143(3), 1119-1125, 2014. https://doi.org/10.1090/S0002-9939-2014-12243-X 
XIN LIU

Institut für Mathematik,

Freie Universität Berlin,

Berlin

Germany.

e-mail: stleonliu@gmail.com

e-mail: stleonliu@live.com

and

EDRISS S. TITI

Department of Mathematics, Texas A\&M University,

College Station TX

77840 USA.

and

EDRISS S. TITI

Department of Applied Mathematics and Theoretical Physics, University of Cambridge,

Cambridge

CB3 0WA UK.

and

EDRISS S. TITI

Department of Computer Science and Applied Mathematics, Weizmann Institute of Science,

76100 Rehovot

Israel.

e-mail: titi@math.tamu.edu

e-mail: Edriss.Titi@damtp.cam.ac.uk

(Received June 6, 2019 / Accepted June 26, 2020)

Published online July 7, 2020

(c) The Author(s) (2020) 